Supporting Information

\title{
Total Syntheses of Fimsbactin A and B and Their Stereoisomers to Probe the Stereoselectivity of the Fimsbactin Uptake Machinery in Acinetobacter baumannii
}

Soojeung Kim, ${ }^{\dagger}$ Haeun Lee, ${ }^{\dagger}$ Woon Young Song ${ }^{\dagger}$ Hak Joong Kim*广,§

${ }^{\dagger}$ Department of Chemistry, Korea University, Seoul 02841, Republic of Korea

${ }^{\S}$ Center for ProteoGenomics Research, Korea University, Seoul 02841, Republic of Korea

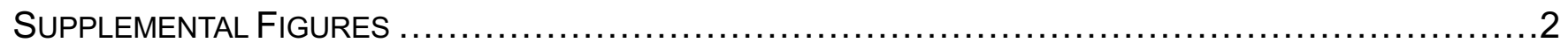

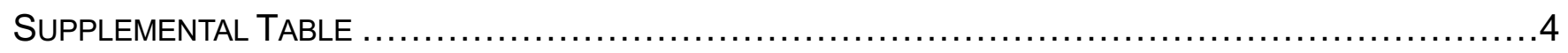

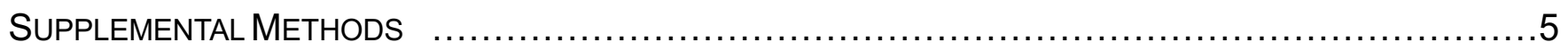

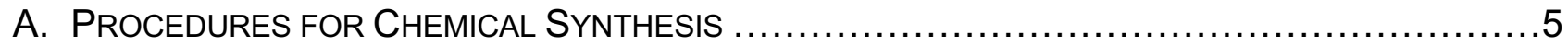

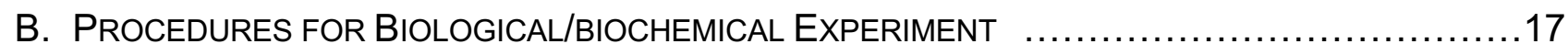

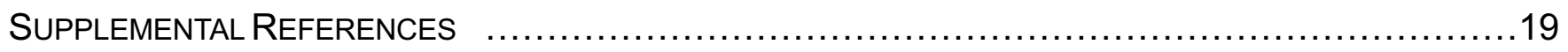

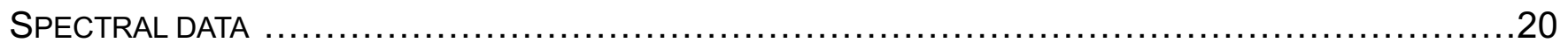




\section{SUPPLEMENTAL FIGURES}
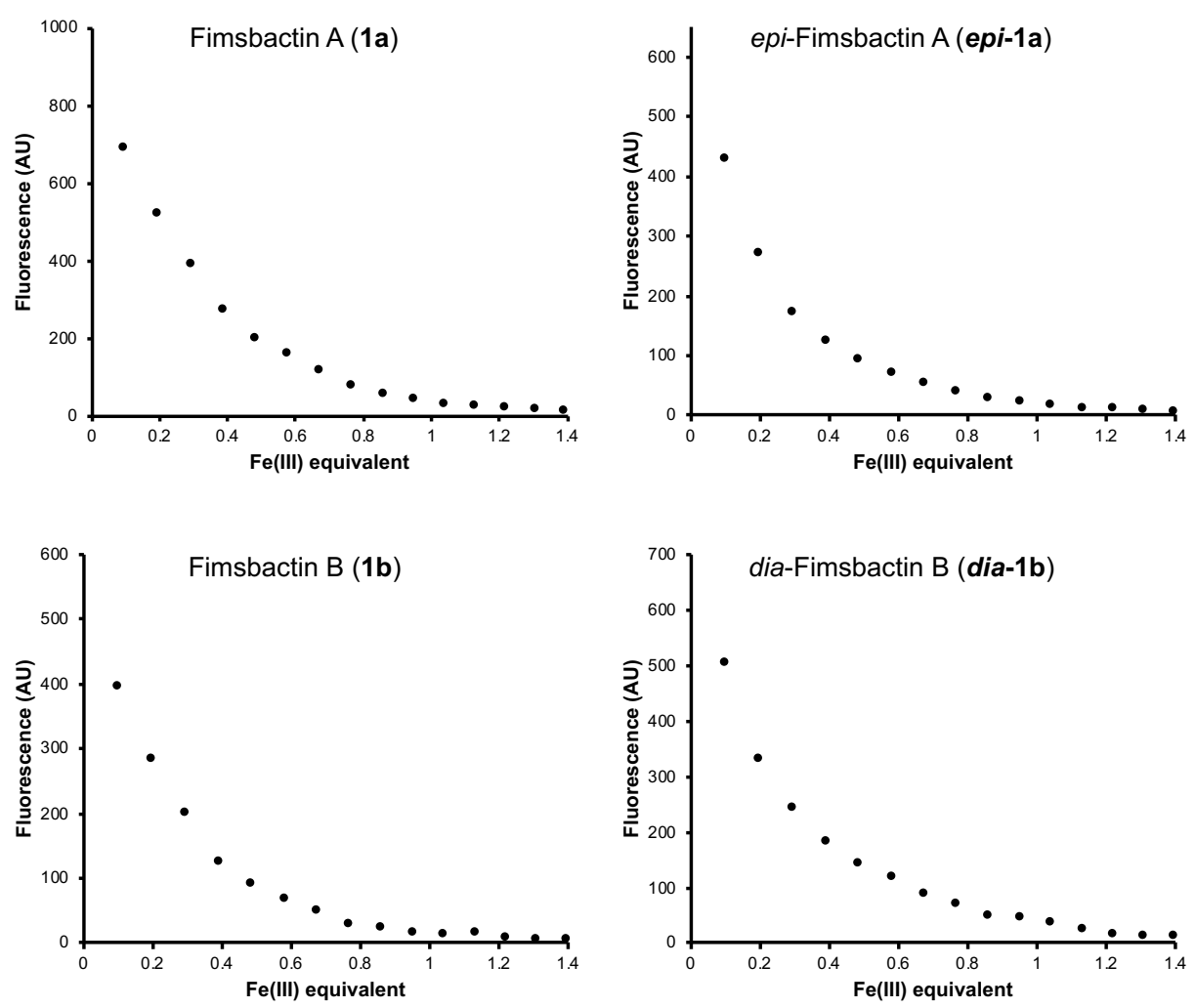

Figure S1. Fluorescence titration of each $500 \mu \mathrm{M}$ fimsbactin in ethanol with $\mathrm{FeCl}_{3}\left(\lambda_{\mathrm{ex}}=320 \mathrm{~nm}, \lambda_{\mathrm{em}}=400 \mathrm{~nm}\right)$. The plot of fluorescence versus Fe(III) equivalent for each fimsbactin was very close to the one previously presented by Bohac et al. ${ }^{\mathrm{S} 5}$, which shows that all fimsbactin isomers are likely bind with $\mathrm{Fe}(\mathrm{III})$ at the 1:1 stoichiometry. Since epi-fimsbactin B (epi-1b) and ent-fimsbactin B (ent-1b) were enantiomers of dia-1 b and $\mathbf{1 b}$, respectively, they were not included in this experiment. 

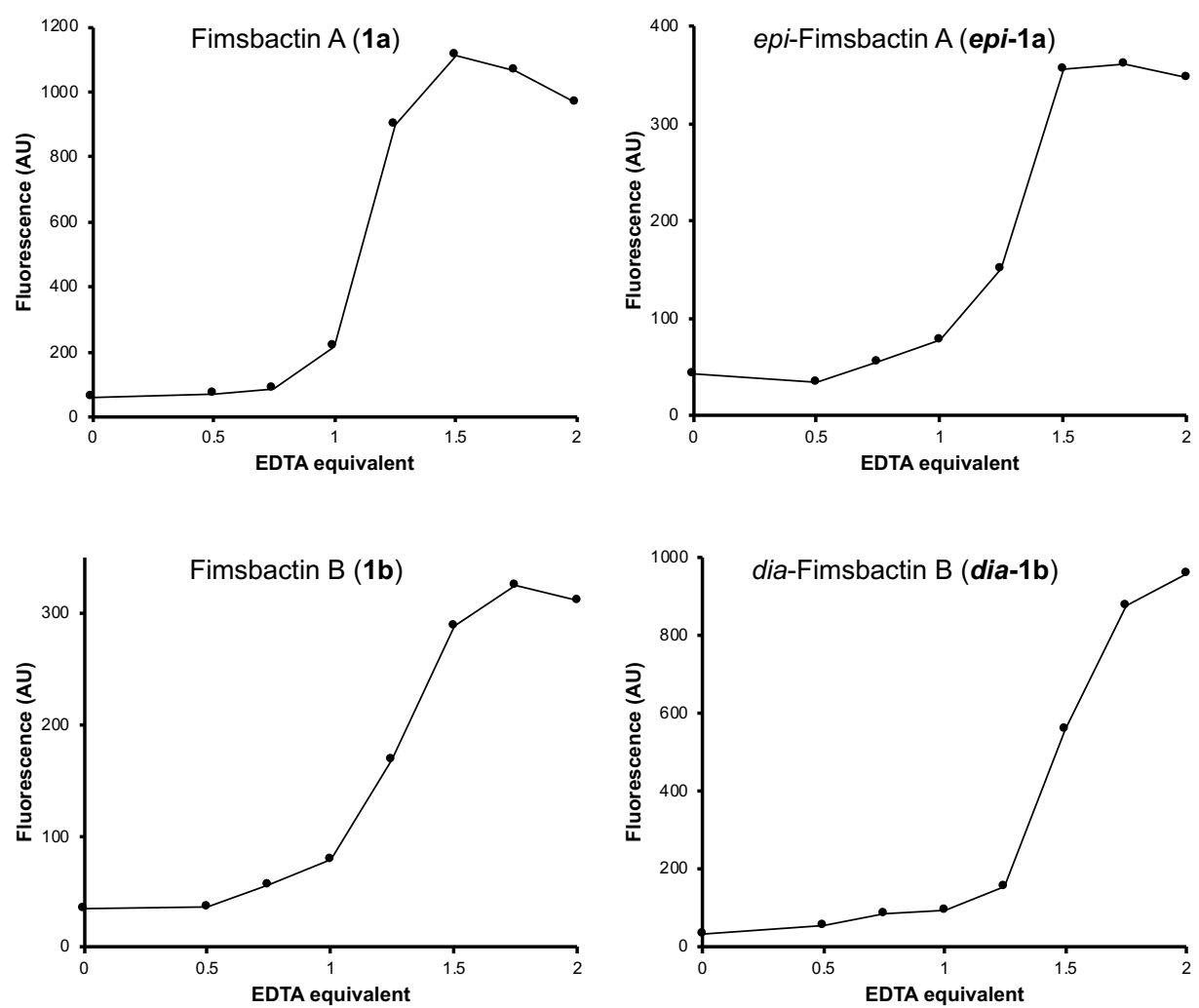

Figure S2. Fluorescence titration of each $500 \mu \mathrm{M}$ Fe(III)-fimsbactin complex in ethanol with EDTA $\left(\lambda_{\mathrm{ex}}=320 \mathrm{~nm}\right.$, $\lambda_{\mathrm{em}}=400 \mathrm{~nm}$ ). Upon a series of additions of EDTA, gradual recovery of the fluorescence corresponding to the release of apo-fimsbactin caused by competition of EDTA was observed. In all cases, discernible fluorescence recovery became visible right after addition of 1 equivalent of EDTA to the similar extent, which indicates that the $\mathrm{Fe}(\mathrm{III})$ affinities of all tested fimsbactin isomers would be comparable to each other. Since epi-fimsbactin B (epi1b) and ent-fimsbactin B (ent-1b) were enantiomers of dia-1b and $\mathbf{1 b}$, respectively, they were not included in this experiment. 


\section{SUPPLEMENTAL TABLE}

\begin{tabular}{|c|c|c|c|c|c|c|c|c|c|}
\hline & \multicolumn{3}{|c|}{${ }^{15} \mathrm{~N}$-labeled Fimsbactin A (reported) ${ }^{\mathrm{S3}}$} & \multicolumn{3}{|c|}{ Fimsbactin A (1a) } & \multicolumn{3}{|c|}{ epi-Fimsbactin A (epi-1a) } \\
\hline Position & ${ }^{13} \mathrm{C}, \mathrm{ppm}$ & ${ }^{1} \mathrm{H}, \mathrm{ppm}$ & $J(\mathrm{~Hz})$ & ${ }^{13} \mathrm{C}, \mathrm{ppm}$ & ${ }^{1} \mathrm{H}, \mathrm{ppm}$ & $J(\mathrm{~Hz})$ & ${ }^{13} \mathrm{C}, \mathrm{ppm}$ & ${ }^{1} \mathrm{H}, \mathrm{ppm}$ & $J(\mathrm{~Hz})$ \\
\hline 1 & 148.2 & & & 148.3 & & & 146.8 & & \\
\hline 2 & 145.7 & & & 145.8 & & & 145.8 & & \\
\hline 3 & 119.3 & 6.968 & $\mathrm{dd}, 1 \mathrm{H}(8.0,1.5)$ & 119.5 & 6.96 & $a p p \mathrm{t}, 1 \mathrm{H}(8.0)$ & 119.4 & 6.97 & $b r \mathrm{~d}, 1 \mathrm{H}(7.4)$ \\
\hline 4 & 118.6 & 6.74 & $\mathrm{t}, 1 \mathrm{H}(8.0)$ & 118.7 & 6.74 & app $\mathrm{t}, 1 \mathrm{H}(7.6)$ & 118.7 & 6.74 & app t, $1 \mathrm{H}(7.8)$ \\
\hline 5 & 117.7 & 7.08 & $\mathrm{dd}, 1 \mathrm{H}(8.0,1.5)$ & 117.9 & 7.09 & $\mathrm{~d}, 1 \mathrm{H}(7.7)$ & 117.9 & 7.08 & $\mathrm{~d}, 1 \mathrm{H}(7.8)$ \\
\hline 6 & 110.1 & & & 110.1 & & & 110.1 & & \\
\hline 7 & 166.3 & & & 166.5 & & & 166.6 & & \\
\hline \multirow[t]{2}{*}{9} & 69.1 & 4.52 & $\mathrm{dd}, 1 \mathrm{H}(8.0,7.5)$ & 69.3 & 4.52 & app t, $1 \mathrm{H}(7.7)$ & 69.4 & 4.46 & $\mathrm{~m}, 1 \mathrm{H}$ \\
\hline & & 4.61 & $\mathrm{dd}, 1 \mathrm{H}(10.0,8.5)$ & & 4.61 & $b r \mathrm{~m}, 1 \mathrm{H}$ & & 4.60 & $b r \mathrm{~m}, 1 \mathrm{H}$ \\
\hline 10 & 66.9 & 5.06 & $\mathrm{dd}, 1 \mathrm{H}(10.0,7.5)$ & 67.2 & 5.07 & $\mathrm{dd}, 1 \mathrm{H}(10.0,7.5)$ & 67.1 & 5.07 & $\mathrm{dd}, 1 \mathrm{H}(10.0,7.5)$ \\
\hline \multicolumn{10}{|l|}{11} \\
\hline 12 & 169.9 & & & 170.1 & & & 170.0 & & \\
\hline 13 & & 8.72 & $\mathrm{dd}, 1 \mathrm{H}(93.0,8.0)$ & & 8.80 & $b r \mathrm{~s}, 1 \mathrm{H}$ & & 8.74 & $b r \mathrm{~s}, 1 \mathrm{H}$ \\
\hline 14 & 51.5 & 4.72 & $\mathrm{~m}, 1 \mathrm{H}$ & 51.7 & 4.71 & $\mathrm{~m}, 1 \mathrm{H}$ & 51.6 & 4.73 & $\mathrm{~m}, 1 \mathrm{H}$ \\
\hline \multirow[t]{2}{*}{15} & 64.3 & 4.39 & $\mathrm{~m}, 1 \mathrm{H}$ & 64.4 & 4.39 & $\mathrm{~m}, 1 \mathrm{H}$ & 64.2 & 4.46 & $\mathrm{~m}, 1 \mathrm{H}$ \\
\hline & & 4.61 & $\mathrm{~m}, 1 \mathrm{H}$ & & 4.57 & $\mathrm{dd}, 1 \mathrm{H}(11,4.7)$ & & 4.46 & $\mathrm{~m}, 1 \mathrm{H}$ \\
\hline 17 & 168.7 & & & 168.9 & & & 168.8 & & \\
\hline 18 & 112.8 & & & 112.7 & & & 112.6 & & \\
\hline 19 & 149.5 & & & 148.3 & & & 148.3 & & \\
\hline 20 & 146.0 & & & 146.2 & & & 146.4 & & \\
\hline 21 & 120.7 & 6.97 & $\mathrm{dd}, 1 \mathrm{H}(8.0,1.5)$ & 120.4 & 6.96 & app t, $1 \mathrm{H}(8.0)$ & 119.9 & 6.97 & $b r \mathrm{~d}, 1 \mathrm{H}(7.4)$ \\
\hline 22 & 118.6 & 6.59 & $\mathrm{t}, 1 \mathrm{H}(8.0)$ & 118.7 & 6.55 & app t, $1 \mathrm{H}(7.6)$ & 118.7 & 6.64 & app $\mathrm{t}, 1 \mathrm{H},(7.8)$ \\
\hline 23 & 119.6 & 7.15 & $\mathrm{dd}, 1 \mathrm{H}(8.0,1.5)$ & 119.8 & 7.14 & $\mathrm{~d}, 1 \mathrm{H}(7.7)$ & 118.8 & 7.18 & $\mathrm{~d}, 1 \mathrm{H}(7.9)$ \\
\hline 24 & 167.7 & & & 167.9 & & & 168.0 & & \\
\hline 25 & & 8.23 & $\mathrm{dt}, 1 \mathrm{H}(92.0,5.5)$ & & 8.31 & $b r \mathrm{~s}, 1 \mathrm{H}$ & & 8.39 & $b r \mathrm{~s}, 1 \mathrm{H}$ \\
\hline 26 & 38.3 & 3.10 & $\mathrm{~m}, 2 \mathrm{H}$ & 38.5 & 3.12 & $\mathrm{~m}, 2 \mathrm{H}$ & 38.5 & 3.11 & $\mathrm{~m}, 2 \mathrm{H}$ \\
\hline 27 & 25.9 & 1.38 & $\mathrm{~m}, 2 \mathrm{H}$ & 26.1 & 1.38 & $\mathrm{~m}, 2 \mathrm{H}$ & 26.0 & 1.37 & $\mathrm{~m}, 2 \mathrm{H}$ \\
\hline 28 & 23.5 & 1.49 & $\mathrm{~m}, 2 \mathrm{H}$ & 23.7 & 1.49 & $\mathrm{~m}, 2 \mathrm{H}$ & 23.7 & 1.48 & $\mathrm{~m}, 2 \mathrm{H}$ \\
\hline 29 & 46.3 & 3.45 & $\mathrm{t}, 2 \mathrm{H}(7.0)$ & 46.5 & 3.45 & $\mathrm{~m}, 2 \mathrm{H}$ & 46.4 & 3.44 & $\mathrm{t}, 2 \mathrm{H}(6.5)$ \\
\hline \multicolumn{10}{|l|}{30} \\
\hline 31 & 170.1 & & & 170.2 & & & 170.2 & & \\
\hline 32 & 20.1 & 1.96 & $\mathrm{~s}, 3 \mathrm{H}$ & 20.4 & 1.96 & $\mathrm{~s}, 3 \mathrm{H}$ & 20.4 & 1.95 & $\mathrm{~s}, 3 \mathrm{H}$ \\
\hline
\end{tabular}

Table S1. Comparison of the NMR spectral data of fimsbactin A (1a) and epi-fimsbactinA (epi-1a). 


\section{SUPPLEMENTAL METHODS}

\section{A. Procedures for Chemical Synthesis}

All reactions were conducted in oven-dried glassware under nitrogen atmosphere with anhydrous solvents, unless otherwise noted. All reactions were monitored by analytical thin-layer chromatography (TLC) using pre-coated silica aluminum plate with F254 indicators, and the product profiles were visualized by UV irradiation $(254 \mathrm{~nm}$, $365 \mathrm{~nm}$ ) and/or staining with a phosphomolybdic acid, ninhydrin, or potassium permanganate solution. The solvents, dichloromethane, toluene, tetrahydrofuran (THF), and N,N-dimethylformate (DMF) were dried by being passed through activated alumina column. Other anhydrous solvents were purchased from Sigma-Aldrich (Missouri, USA) or Acros Organics (Belgium). All chemical reagents used in the reactions were purchased from Sigma-Aldrich, Acros Organics, TCI Chemicals (Japan), Alfa-Aesar (Massachusettes, USA), AK Scientific (California, USA), or Daejung Chemicals \& Metals (Republic of Korea), and they were used as received unless otherwise noted. ${ }^{1} \mathrm{H}-$ NMR and ${ }^{13} \mathrm{C}-\mathrm{NMR}$ data were recorded using Varian Unity $400(400 / 100 \mathrm{MHz})$ or Bruker Advance 500 (500/125 $\mathrm{MHz})$. Chemical shifts were reported in parts per million (ppm) relative to chloroform $\left({ }^{1} \mathrm{H}: 7.26 \mathrm{ppm},{ }^{13} \mathrm{C}: 77.0\right.$ ppm), methanol ( $\left.{ }^{1} \mathrm{H}: 3.31 \mathrm{ppm},{ }^{13} \mathrm{C}: 49.05 \mathrm{ppm}\right), \mathrm{D}_{2} \mathrm{O}\left({ }^{1} \mathrm{H}: 4.65 \mathrm{ppm}\right)$, or tetramethylsilane (TMS, $\left.0.00 \mathrm{ppm}\right)$, and the coupling constants were reported in Herz $(\mathrm{Hz})$. High-resolution mass spectra were collected using Bruker Compact QTOF, where the electrospray ionization method was employed for ionization.

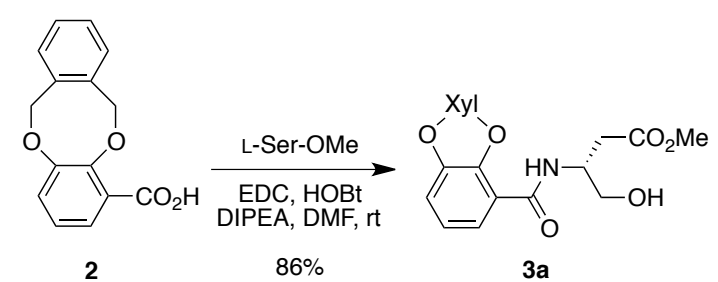

Compound 3a. To a solution of acid $2^{\mathrm{S} 1}(2.170 \mathrm{~g}, 8.468 \mathrm{mmol})$, L-Ser-OMe $\cdot \mathrm{HCl}(1.970 \mathrm{~g}, 12.70 \mathrm{mmol})$, and $N, N$ diisopropylethylamine (DIPEA, $4.4 \mathrm{ml}, 25.40 \mathrm{mmol})$ in $N, N$-dimethylformamide (DMF, $42 \mathrm{~mL}$ ) was added $N$-(3dimethylaminopropyl)- $N$ '-ethylcarbodiimide (EDC, $3.25 \mathrm{~g}, 16.93 \mathrm{mmol}$ ) and 1-hydroxybenzotriazole hydrate (HOBt, $2.59 \mathrm{~g}, 16.93 \mathrm{mmol}$ ) at $0^{\circ} \mathrm{C}$. After the temperature was elevated to room temperature, the stirring continued for $3 \mathrm{hr}$. Upon confirmation of complete consumption of 2, the solvent was removed under reduced pressure, and the resulting residue was diluted with ethyl acetate $(250 \mathrm{~mL})$. The organic phase was sequentially washed with $1 \mathrm{M}$ $\mathrm{HCl}$ solution, saturated sodium bicarbonate solution, and brine. The solution was dried over anhydrous magnesium sulfate and concentrated under reduced pressure to afford the crude product in a solid form. After washed with petroleum ether, the solid was resuspended in dichloromethane, and the resulting solution was washed with $1 \mathrm{M}$ $\mathrm{HCl}$ solution and saturated sodium bicarbonate solution in sequence. After concentration under reduced pressure, the desired amide $3 \mathrm{a}(2.768 \mathrm{~g}, 7.282 \mathrm{mmol})$ was obtained in $86 \%$ yield with no further purification. ${ }^{1} \mathrm{H}$ NMR (500 
$\left.\mathrm{MHz}, \mathrm{CDCl}_{3}\right) \delta 9.14(\mathrm{~d}, J=6.7 \mathrm{~Hz}, 1 \mathrm{H}), 7.78(\mathrm{dd}, J=7.9,1.8 \mathrm{~Hz}, 1 \mathrm{H}), 7.33-7.29(\mathrm{~m}, 1 \mathrm{H}), 7.28-7.25(\mathrm{~m}, 2 \mathrm{H})$, $7.20(\mathrm{dd}, J=7.9,1.8 \mathrm{~Hz}, 1 \mathrm{H}), 7.12(\mathrm{dd}, J=5.2,3.6 \mathrm{~Hz}, 1 \mathrm{H}), 6.98(\operatorname{app} \mathrm{t}, J=7.9 \mathrm{~Hz}, 1 \mathrm{H}), 5.68(\mathrm{~d}, J=12.3 \mathrm{~Hz}, 1 \mathrm{H})$, $5.58(\mathrm{~d}, J=12.3 \mathrm{~Hz}, 1 \mathrm{H}), 5.40(\mathrm{~d}, J=13.7 \mathrm{~Hz}, 1 \mathrm{H}), 5.31(\mathrm{~d}, J=13.7 \mathrm{~Hz}, 1 \mathrm{H}), 4.86(\mathrm{dt}, J=7.2,3.7 \mathrm{~Hz}, 1 \mathrm{H}), 4.05$ $-4.03(\mathrm{~m}, 2 \mathrm{H}), 3.82(\mathrm{~s}, 3 \mathrm{H}) .{ }^{13} \mathrm{C}$ NMR $\left(125 \mathrm{MHz}, \mathrm{CDCl}_{3}\right) \delta 171.3,165.4,150.2,149.6,136.5,133.9,130.3,129.3$, 128.8, 128.0, 126.7, 126.3, 124.7, 123.1, 76.5, 75.2, 63.8, 55.6, 55.9. HR-MS (ESI-TOF) $m / z$ for $\left[\mathrm{C}_{19} \mathrm{H}_{19} \mathrm{NNaO}_{6}\right]^{+}$ $\left([\mathrm{M}+\mathrm{Na}]^{+}\right)$: calculated 380.1110 , found 380.1107 .

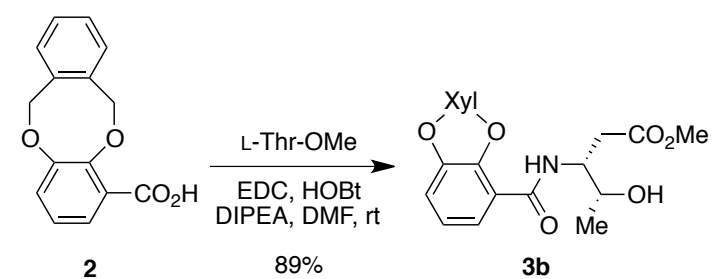

Compound 3b. Amide 3b was prepared by following the previously established synthetic method ${ }^{\mathrm{S} 1}$ from acid 2 using L-Thr-OMe in $89 \%$ yield. The ${ }^{1} \mathrm{H}-,{ }^{13} \mathrm{C}-\mathrm{NMR}$ and HR-MS spectra of compound $\mathbf{3 b}$ were reported in Ref. S1.

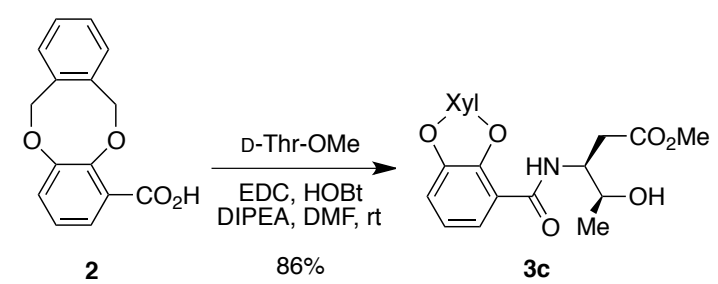

Compound 3c. Amide 3c was prepared following the method used for synthesis of amide $\mathbf{3} \mathbf{b}^{\mathrm{S} 1}$ except for the use of D-Thr-OMe instead of L-Thr-OMe, and the yield was $86 \%$. The ${ }^{1} \mathrm{H}-,{ }^{13} \mathrm{C}-\mathrm{NMR}$, and HR-MS spectra of compound $\mathbf{3 c}$ were identical to those of its enantiomer, compound $\mathbf{3 b}$.

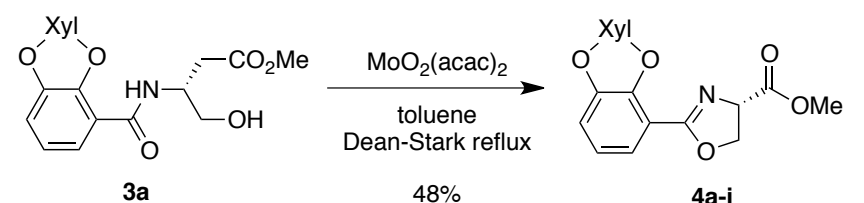

Compound 4a-i. The reaction was carried out in a flask fitted with a pressure-equalized addition funnel (containing a cotton plug and molecular sieves to function as a Soxhlet extractor) surmounted with a reflux condenser. A solution of amide 3a $(0.512 \mathrm{~g}, 1.491 \mathrm{mmol})$ and bis(acetylacetonato)dioxomolybdenum(VI) $\left(\mathrm{MoO}_{2}(\mathrm{acac})_{2}, 48.6 \mathrm{mg}, 10\right.$ $\mathrm{mol} \%)$ in toluene $(150 \mathrm{~mL})$ was heated with an oil bath under azeotropic reflux. After $8 \mathrm{~h}$, the reaction mixture was cooled to the ambient temperature and concentrated under reduced pressure. The crude product was subjected to flash column chromatography $\left(\mathrm{SiO}_{2}\right.$, hexanes:etheyl acetate $\left.=9: 1\right)$ to yield the desired ester 4a-i $(0.242 \mathrm{~g}, 0.710$ $\mathrm{mmol})$ in $48 \%$ yield. ${ }^{1} \mathrm{H}$ NMR $\left(500 \mathrm{MHz}, \mathrm{CDCl}_{3}\right) \delta 7.43(\mathrm{dd}, J=7.8,1.7 \mathrm{~Hz}, 1 \mathrm{H}) .7 .26-7.24(\mathrm{~m}, 2 \mathrm{H}), 7.20-7.19$ 
(m, 1H), $7.17-7.15(\mathrm{~m}, 1 \mathrm{H}), 7.10(\mathrm{dd}, J=8,1.7 \mathrm{~Hz}, 1 \mathrm{H}), 6.93(\mathrm{t}, 7.9 \mathrm{~Hz}, 1 \mathrm{H}), 5.45(\mathrm{~d}, J=12.4 \mathrm{~Hz}, 1 \mathrm{H}), 5.44$ (d, $J=5.1 \mathrm{~Hz}, 2 \mathrm{H}), 5.38(\mathrm{~d}, J=12.8 \mathrm{~Hz}, 1 \mathrm{H}), 4.97(\mathrm{dd}, J=10.6,7.9 \mathrm{~Hz}, 1 \mathrm{H}), 4.66(\mathrm{t}, J=8.3 \mathrm{~Hz}, 1 \mathrm{H}), 4.58(\mathrm{dd}, J=$ 10.7, $8.7 \mathrm{~Hz}, 1 \mathrm{H}), 3.82(\mathrm{~s}, 3 \mathrm{H}) .{ }^{13} \mathrm{C}$ NMR $\left(125 \mathrm{MHz}, \mathrm{CDCl}_{3}\right) \delta 171.9,165.2,151.5,149.6,136.0,135.4,129.3$, $128.7,128.6,128.5,125.2,124.9,123.5,121.5,76.0,74.9,69.5,68.8,52.7$. HR-MS (ESI-TOF) $m / z$ for $\left[\mathrm{C}_{19} \mathrm{H}_{18} \mathrm{NO}_{5}\right]^{+}\left([\mathrm{M}+\mathrm{H}]^{+}\right)$: calculated 340.1185, found 340.1192.

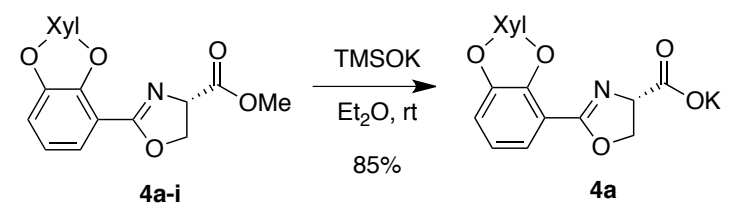

Compound 4a. Ester 4a-i (0.242 g, $0.710 \mathrm{mmol})$ was dissolved in ethyl ether $(20 \mathrm{~mL})$ and treated with potassium trimethylsilanolate $(0.288 \mathrm{~g}, 0.852 \mathrm{mmol})$ at room temperature. After stirred for $3 \mathrm{hr}$, the resulting solid was collected by filtration and washed with hexane/ethyl ether $(1: 1)$. The crude product was dried under reduced pressure to afford the desired potassium carboxylate $4 \mathbf{a}(0.199 \mathrm{~g}, 0.61 \mathrm{mmol})$ in $85 \%$ yield. The product was used in the next step without further purification. ${ }^{1} \mathrm{H}$ NMR $\left(500 \mathrm{MHz}\right.$, methanol- $\left.d^{4}\right) \delta 7.38(\mathrm{dd}, J=7.8,1.7 \mathrm{~Hz}, 1 \mathrm{H}), 7.28$ $-7.25(\mathrm{~m}, 2 \mathrm{H}), 7.27-7.22(\mathrm{~m}, 2 \mathrm{H}), 7.08$ (dd, $J=8.1,1.7 \mathrm{~Hz}, 1 \mathrm{H}), 6.94$ (app t, $J=7.9 \mathrm{~Hz}, 1 \mathrm{H}), 5.49$ (d, $J=13.2$ $\mathrm{Hz}, 1 \mathrm{H}), 5.44(\mathrm{~d}, J=13.2 \mathrm{~Hz}, 1 \mathrm{H}), 5.43(\mathrm{~d}, J=2.3 \mathrm{~Hz}, 2 \mathrm{H}), 4.77(\mathrm{dd}, J=10.6,8.5 \mathrm{~Hz}, 1 \mathrm{H}), 4.62(\mathrm{dd}, J=10.6,8.2$ $\mathrm{Hz}, 1 \mathrm{H}), 4.52(\mathrm{t}, J=8.4 \mathrm{~Hz}, 1 \mathrm{H}), 1.90(\mathrm{~s}, 3 \mathrm{H}) .{ }^{13} \mathrm{C}$ NMR $\left(125 \mathrm{MHz}\right.$, methanol- $\left.d^{4}\right) \delta 180.4,178.8,165.2,152.3$, $150.3,137.2,136.9,130.4,130.0,129.8,129.7,126.2,125.8,124.4,123.4,76.2,75.7,72.4,72.2$. HR-MS (ESITOF) $m / z$ for $\left[\mathrm{C}_{18} \mathrm{H}_{16} \mathrm{NO}_{5}\right]^{+}\left([\mathrm{M}+\mathrm{H}]^{+}\right)$: calculated 326.1028 , found 326.1023 .

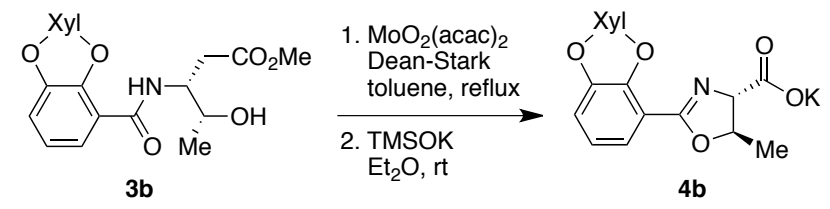

Compound $\mathbf{4 b}$. The synthesis of compound $\mathbf{4 b}$ from $\mathbf{3 b}$ was previously reported in Ref S1.

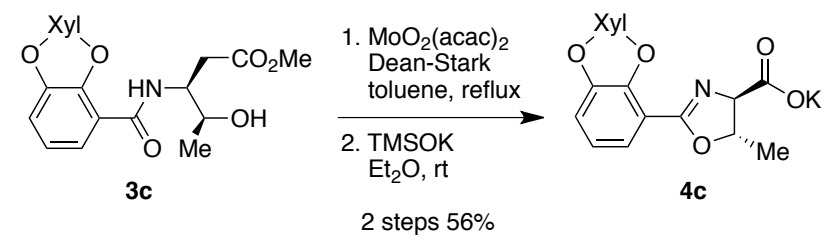

Compound 4c. Potassium carboxylate $4 \mathbf{c}$ was prepared from $3 \mathbf{c}$ following the method used for synthesis of amide 4a, and the two-step yield was $56 \%$. The ${ }^{1} \mathrm{H}-,{ }^{13} \mathrm{C}-\mathrm{NMR}$, and HR-MS spectra of compound $\mathbf{4 c}$ were identical to those of its enantiomer, compound $\mathbf{4 b}$ reported in Ref $\mathrm{S} 1$. 


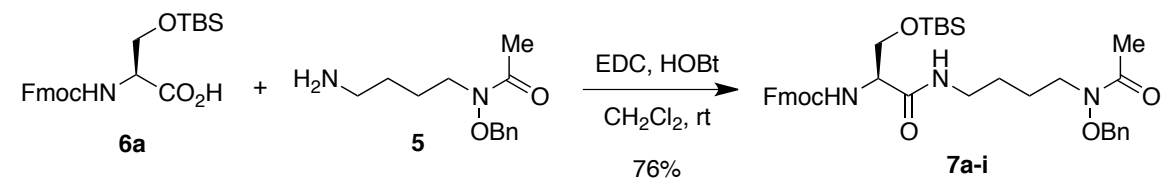

Compound 7a-i. To a solution of the amine $5^{\mathrm{S} 2}$ (3.00 g, $\left.10.2 \mathrm{mmol}\right)$ and Fmoc-L-Ser(TBS)-OH $6 \mathbf{6}$ (4.60 g, 12.2 $\mathrm{mmol})$ in dichloromethane $(60 \mathrm{~mL})$ was added EDC $(2.33 \mathrm{~g}, 12.2 \mathrm{mmol})$ and $\mathrm{HOBt}(1.65 \mathrm{~g}, 12.2 \mathrm{mmol})$ at $0{ }^{\circ} \mathrm{C}$. After stirred for $3 \mathrm{hr}$ at room temperature, the reaction mixture was diluted with ethyl acetate $(50 \mathrm{~mL})$ and poured into $1 \mathrm{~N} \mathrm{HCl}$ solution $(100 \mathrm{~mL})$. After collecting the organic layer, the aqueous layer was further extracted with ethyl acetate $(100 \mathrm{~mL} \times 2)$. The combined organic layers were washed with saturated sodium bicarbonate solution and brine, dried over anhydrous magnesium sulfate, and concentrated under reduced pressure. The crude residue was subjected to flash column chromatography $\left(\mathrm{SiO}_{2}, 2 \%\right.$ methanol in dichloromethane) to afford the desired Fmocamine intermediate 7a-i (5.12 g, $7.75 \mathrm{mmol})$ in 76\% yield. ${ }^{1} \mathrm{H} \mathrm{NMR}\left(500 \mathrm{MHz}, \mathrm{CDCl}_{3}\right) \delta 7.76(\mathrm{~d}, J=7.5 \mathrm{~Hz}, 2 \mathrm{H})$, $7.59(b r \mathrm{t}, J=6.5 \mathrm{~Hz}, 2 \mathrm{H}), 7.42-7.34(\mathrm{~m}, 7 \mathrm{H}), 7.31(\mathrm{td}, J=7.5,1.0 \mathrm{~Hz}, 2 \mathrm{H}), 6.66(b r \mathrm{~s}, 1 \mathrm{H}), 5.77(b r \mathrm{~s}, 1 \mathrm{H}), 4.79$ $(\mathrm{s}, 2 \mathrm{H}), 4.40(\mathrm{~d}, J=7.4 \mathrm{~Hz}, 2 \mathrm{H}), 4.22(\mathrm{t}, J=7.1 \mathrm{~Hz}, 1 \mathrm{H}), 4.16(b r \mathrm{~s}, 1 \mathrm{H}), 4.02(\mathrm{dd}, J=9.8,3.9 \mathrm{~Hz}, 1 \mathrm{H}), 3.68-3.60$ $(\mathrm{m}, 3 \mathrm{H}), 3.35-3.24(\mathrm{~m}, 2 \mathrm{H}), 2.08(\mathrm{~s}, 3 \mathrm{H}), 1.70-1.63(\mathrm{~m}, 2 \mathrm{H}), 1.55-1.47(\mathrm{~m}, 2 \mathrm{H}), 0.89(\mathrm{~s}, 9 \mathrm{H}), 0.08(\mathrm{~s}, 3 \mathrm{H}), 0.07$ (s, 3H). ${ }^{13} \mathrm{C}$ NMR $\left(125 \mathrm{MHz}, \mathrm{CDCl}_{3}\right) \delta 172.4,170.1,156.1,143.9,143.8,141.37,141.36,134.4,129.3,129.1$, 128.8, 127.8, 127.1, 125.18, 125.16, 120.1, 76.4, 67.2, 63.3, 55.8, 47.2, 44.8, 39.3, 26.6, 25.9, 24.4, 20.5, 18.2, 5.4, -5.5. HR-MS (ESI-TOF) $m / z$ for $\left[\mathrm{C}_{37} \mathrm{H}_{49} \mathrm{~N}_{3} \mathrm{NaO}_{6} \mathrm{Si}\right]^{+}\left([\mathrm{M}+\mathrm{Na}]^{+}\right)$: calculated 682.3288 , found 682.3286 .

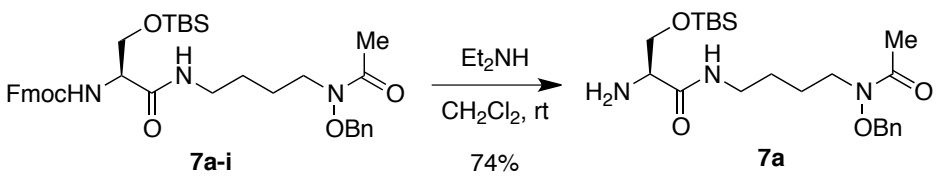

Compound 7a. A solution of 7a-i $(5.12 \mathrm{~g}, 7.75 \mathrm{mmol})$ in dichloromethane $(40 \mathrm{~mL})$ was treated with diethylamine $(20 \mathrm{~mL})$ at room temperature. After stirred for $6 \mathrm{hr}$, the crude product was dried under reduced pressure. The crude product was subjected to flash column chromatography $\left(\mathrm{SiO}_{2}, 10 \%\right.$ methanol in dichloromethane) to afford the desired amine $7 \mathbf{a}(2.50 \mathrm{~g}, 5.71 \mathrm{mmol})$ in $74 \%$ yield. ${ }^{1} \mathrm{H}$ NMR $\left(500 \mathrm{MHz}, \mathrm{CDCl}_{3}\right) \delta 7.48(b r \mathrm{t}, J=4.6 \mathrm{~Hz}, 1 \mathrm{H}), 7.40$ - $7.34(\mathrm{~m}, 4 \mathrm{H}), 4.80(\mathrm{~s}, 2 \mathrm{H}), 3.82(\mathrm{dd}, J=9.9,4.6 \mathrm{~Hz}, 1 \mathrm{H}), 3.77(\mathrm{dd}, J=9.9,6.1 \mathrm{~Hz}, 1 \mathrm{H}), 3.68-3.63(b r \mathrm{~m}, 2 \mathrm{H})$, $3.46(\mathrm{dd}, J=5.9,4.7 \mathrm{~Hz}, 1 \mathrm{H}), 3.25(\mathrm{q}, J=6.9 \mathrm{~Hz}, 2 \mathrm{H}), 2.09(\mathrm{~s}, 3 \mathrm{H}), 1.69-1.63(\mathrm{~m}, 2 \mathrm{H}), 1.54-1.48(\mathrm{~m}, 2 \mathrm{H}), 0.87$ (s, 9H), 0.06 (s, 3H), 0.05 (s, 3H). ${ }^{13} \mathrm{C}$ NMR $\left(125 \mathrm{MHz}, \mathrm{CDCl}_{3}\right) \delta 172.9,172.2,134.3,129.1,128.9,128.6,76.2$, 65.3, 56.5, 50.1, 44.7, 38.5, 26.7, 25.7, 24.2, 20.4, 18.1, -5.5, -5.6. HR-MS (ESI-TOF) $m / z$ for $\left[\mathrm{C}_{22} \mathrm{H}_{40} \mathrm{~N}_{3} \mathrm{O}_{4} \mathrm{Si}^{+}\right.$ $\left([\mathrm{M}+\mathrm{H}]^{+}\right)$: calculated 438.2788 , found 438.2788 . 


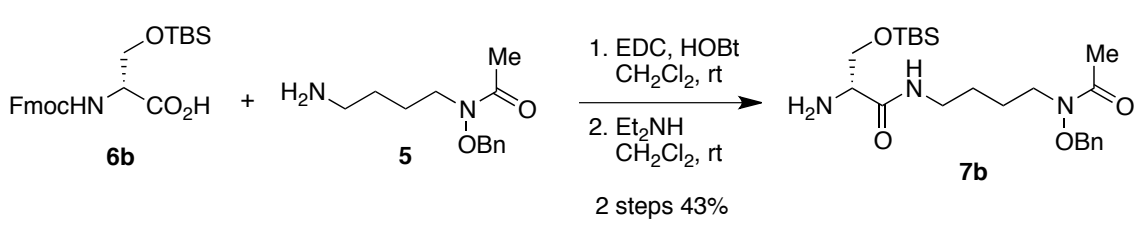

Compound 7b. Compound $\mathbf{7 b}$ was synthesized analogously to preparation of compound 7a from amine $\mathbf{5}$ using Fmoc-D-Ser(TBS)-OH. The two-step yield was $43 \%$, and the ${ }^{1} \mathrm{H}-,{ }^{13} \mathrm{C}-\mathrm{NMR}$, and HR-MS spectra of compound $7 \mathbf{b}$ were identical to those of its enantiomer, compound $7 \mathbf{a}$.
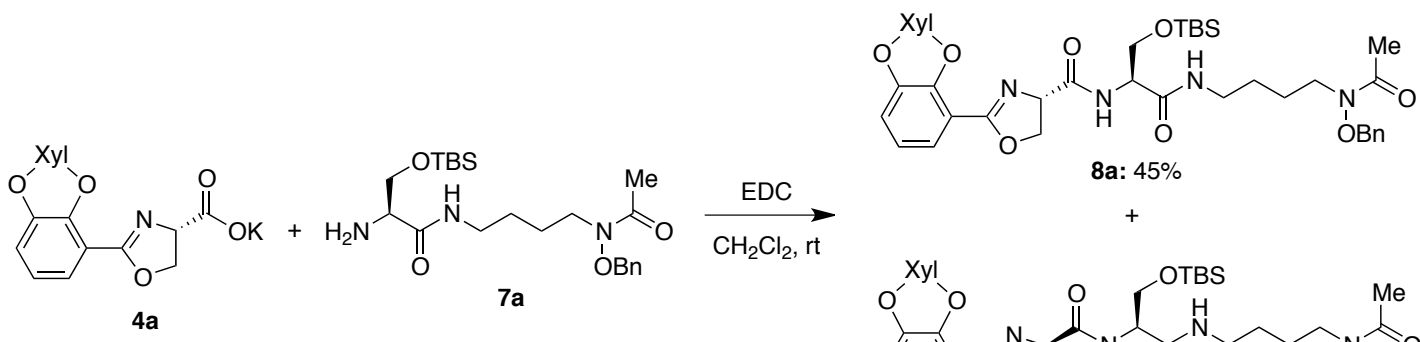

8 a: $45 \%$

$+$

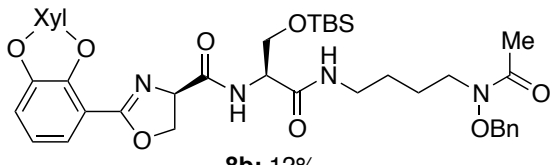

8b: $12 \%$

Compound 8a/8b (the condition in which epimerization was observed). To a solution of amine $7 \mathbf{a}(0.310 \mathrm{~g}, 0.711$ $\mathrm{mmol})$ and potassium carboxylate $4 \mathbf{a}(0.387 \mathrm{~g}, 1.07 \mathrm{mmol})$ in dichloromethane $(10 \mathrm{~mL})$ was added EDC $(0.204 \mathrm{~g}$, $1.07 \mathrm{mmol}$ ) at $0{ }^{\circ} \mathrm{C}$. The reaction mixture was stirred at room temperature for $5 \mathrm{hr}$, and then it was diluted with dichloromethane $(25 \mathrm{~mL})$ and $1 \mathrm{~N} \mathrm{HCl}$ solution $(25 \mathrm{~mL})$. After collection of the organic layer, the aqueous layer was extracted with dichloromethane $(20 \mathrm{~mL} \times 2)$. The combined organic layers were then washed with saturated sodium bicarbonate solution and brine, dried over anhydrous magnesium sulfate, and concentrated under reduced pressure. In the crude residue, two discernible spots suspected to be diastereomers were detected at $R_{f}=0.28$ and 0.31 based on the thin layer chromatography analysis (5\% methanol in dichloromethane). This mixture was separated by flash column chromatography $\left(\mathrm{SiO}_{2}, 3 \%\right.$ methanol in dichloromethane) to give the desired amide $8 \mathbf{a}$ $(0.258 \mathrm{~g}, 0.320 \mathrm{mmol})$ and its diastereomer $\mathbf{8 b}(0.0 .63 \mathrm{~g}, 0.085 \mathrm{mmol})$ in $45 \%$ and $12 \%$ yields, respectively. 8a: ${ }^{1} \mathrm{H}$ NMR (500 MHz, methanol- $\left.d^{4}\right) \delta 7.44-7.35(\mathrm{~m}, 6 \mathrm{H}), 7.31-7.27(\mathrm{~m}, 3 \mathrm{H}), 7.23(\mathrm{~d}, J=7.0 \mathrm{~Hz}, 1 \mathrm{H}), 7.17(\mathrm{~d}, J=8.0$ $\mathrm{Hz}, 1 \mathrm{H}), 6.96(\mathrm{t}, J=7.9 \mathrm{~Hz}, 1 \mathrm{H}), 5.53(\mathrm{~s}, 1 \mathrm{H}), 5.48(\mathrm{~s}, 2 \mathrm{H}), 5.43(\mathrm{~d}, J=13.1 \mathrm{~Hz}, 1 \mathrm{H}), 5.39(\mathrm{~d}, J=13.0 \mathrm{~Hz}, 1 \mathrm{H})$, $4.96(\mathrm{dd}, J=10.6,8.0 \mathrm{~Hz}, 2 \mathrm{H}), 4.88(\mathrm{~s}, 2 \mathrm{H}), 4.64(\operatorname{app~t}, J=9.9 \mathrm{~Hz}, 1 \mathrm{H}), 4.50(\operatorname{app~t}, J=8.2 \mathrm{~Hz}, 1 \mathrm{H}), 4.44(b r \mathrm{t}, J$ $=4.2 \mathrm{~Hz}, 1 \mathrm{H}), 3.93(\mathrm{dd}, J=10.0,3.9 \mathrm{~Hz}, 1 \mathrm{H}), 3.81(\mathrm{dd}, J=10.0,4.6 \mathrm{~Hz}, 2 \mathrm{H}), 3.73-3.65(\mathrm{~m}, 2 \mathrm{H}), 3.25(b r \mathrm{t}, J=$ $6.0 \mathrm{~Hz}, 2 \mathrm{H}) 2.04(\mathrm{~s}, 3 \mathrm{H}), 1.70-1.63(\mathrm{~m}, 2 \mathrm{H}), 1.55-1.48(\mathrm{~m}, 2 \mathrm{H}), 0.76(\mathrm{~s}, 9 \mathrm{H}),-0.04(\mathrm{~s}, 3 \mathrm{H}),-0.06(\mathrm{~s}, 3 \mathrm{H}) .{ }^{13} \mathrm{C}$ NMR $\left(125 \mathrm{MHz}\right.$, methanol- $\left.d^{4}\right) \delta 173.8,171.7,171.6,166.6,152.5,151.2,137.1,136.9,136.0,130.7,130.3,130.0$, 129.9, 129.8, 129.7, 129.7, 126.7, 126.3, 124.2, 122.0, 77.1, 76.33, 76.25, 71.1, 70.3, 64.4, 56.3, 40.1, 27.6, 26.2, 25.2, 20.4, 19.0, -5.39, -5.44. HR-MS (ESI-TOF) $m / z$ for $\left[\mathrm{C}_{40} \mathrm{H}_{53} \mathrm{~N}_{4} \mathrm{O}_{8} \mathrm{Si}\right]^{+}\left([\mathrm{M}+\mathrm{H}]^{+}\right)$: calculated 745.3633, found 745.3630. 8b: ${ }^{1} \mathrm{H}$ NMR $\left(500 \mathrm{MHz}\right.$, methanol- $\left.d^{4}\right) \delta 7.43-7.35(\mathrm{~m}, 6 \mathrm{H}), 7.30-7.22(\mathrm{~m}, 4 \mathrm{H}), 7.15(\mathrm{dd}, J=8.1,1.7$ $\mathrm{Hz}, 1 \mathrm{H}), 6.97$ (t, $J=7.9 \mathrm{~Hz}, 1 \mathrm{H}), 5.54(\mathrm{~d}, J=13.1 \mathrm{~Hz}, 1 \mathrm{H}), 5.49(\mathrm{~d}, J=13.1 \mathrm{~Hz}, 1 \mathrm{H}), 5.44(\mathrm{~d}, J=13.0 \mathrm{~Hz}, 1 \mathrm{H})$, 
$5.41(\mathrm{~d}, J=13.0 \mathrm{~Hz}, 1 \mathrm{H}), 4.96(\mathrm{dd}, J=10.9,8.0 \mathrm{~Hz}, 1 \mathrm{H}), 4.84(\mathrm{~s}, 2 \mathrm{H}), 4.65(\mathrm{dd}, J=10.9,8.6 \mathrm{~Hz}, 1 \mathrm{H}), 4.55$ (app t, $J=8.3 \mathrm{~Hz}, 1 \mathrm{H}), 4.45(\operatorname{app} \mathrm{t}, J=4.9 \mathrm{~Hz}, 1 \mathrm{H}), 3.96(\mathrm{dd}, J=10.1,4.6 \mathrm{~Hz}, 1 \mathrm{H}), 3.89(\mathrm{dd}, J=10.1,5.3 \mathrm{~Hz}, 1 \mathrm{H}), 3.21$ (t, $J=6.9 \mathrm{~Hz}, 2 \mathrm{H}), 2.00(\mathrm{~s}, 3 \mathrm{H}), 1.66-1.58(\mathrm{~m}, 2 \mathrm{H}), 1.51-1.44(\mathrm{~m}, 2 \mathrm{H}), 0.88(\mathrm{~s}, 9 \mathrm{H}), 0.07(\mathrm{~s}, 3 \mathrm{H}), 0.06(\mathrm{~s}, 3 \mathrm{H})$. ${ }^{13} \mathrm{C}$ NMR $\left(125 \mathrm{MHz}\right.$, methanol- $\left.d^{4}\right) \delta 173.7,171.62,171.58,166.6,152.5,150.9,137.07,137.02,136.1,130.7,130.3$, $130.1,130.00,129.81,129.79,129.7,126.6,126.3,124.3,122.3,77.1,76.4,76.1,70.8,70.3,64.4,56.5,40.1,27.5$, 26.3, 25.3, 20.4, 19.1, -5.33, -5.35. HR-MS (ESI-TOF) $m / z$ for $\left[\mathrm{C}_{40} \mathrm{H}_{53} \mathrm{~N}_{4} \mathrm{O}_{8} \mathrm{Si}^{+}\left([\mathrm{M}+\mathrm{H}]^{+}\right)\right.$: calculated 745.3633, found 745.3630 .

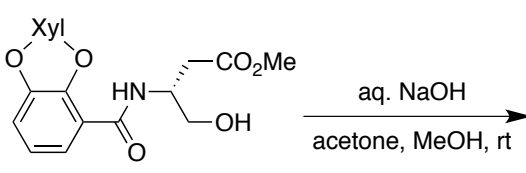

$3 \mathbf{a}$

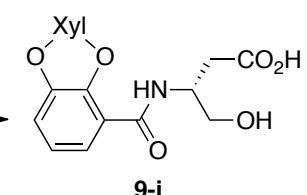

9-i

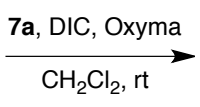

2 steps $48 \%$

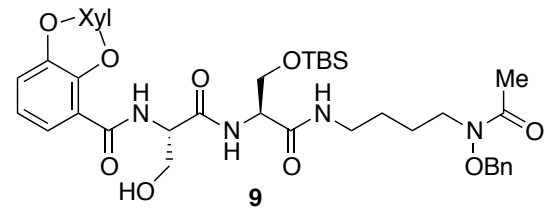

Compound 9. To a solution of $3 \mathbf{a}(2.27 \mathrm{~g}, 6.36 \mathrm{mmol})$ in acetone $(15 \mathrm{~mL})$ and methanol $(15 \mathrm{~mL})$ was added an aqueous solution of sodium hydroxide $(1.0 \mathrm{M}, 15 \mathrm{~mL})$ at $0{ }^{\circ} \mathrm{C}$, and the mixture was stirred for 30 min at $0{ }^{\circ} \mathrm{C}$. After stirred for $1 \mathrm{hr}$ at room temperature, the reaction mixture was cooled to $0^{\circ} \mathrm{C}$ and acidified to $\mathrm{pH} 2$ with concentrated hydrochloric acid. Then, acetone and methanol were removed under reduced pressure, and the resulting aqueous layer was diluted with water followed by extraction with ethyl acetate $(3 \times 20 \mathrm{~mL})$. The combined organic layers were washed with brine, dried over magnesium sulfate, and concentrated under reduced pressure. The resulting acid 9-i (crude $249 \mathrm{mg}, 0.686 \mathrm{mmol}$ ) was used in the next step without further purification. Acid 9-i was mixed with amine 7a $(250 \mathrm{mg}, 0.572 \mathrm{mmol})$ in dichloromethane $(10 \mathrm{~mL})$, and this mixture was treated with diisopropylcarbodiimide (DIC, $144 \mathrm{mg}, 1.14 \mathrm{mmol}$ ), and Oxyma ${ }^{\circledR}(163 \mathrm{mg}, 1.14 \mathrm{mmol}$ ) at room temperature. After stirred for $3 \mathrm{hr}$, the reaction mixture was diluted with dichloromethane $(30 \mathrm{~mL})$. The organic layer was washed with saturated sodium bicarbonate solution and brine, dried over anhydrous magnesium sulfate, and concentrated under reduced pressure. The crude residue was subjected to flash column chromatography $\left(\mathrm{SiO}_{2}, 2 \%\right.$ methanol in dichloromethane) to afford the desired product $9(567 \mathrm{mg}, 1.06 \mathrm{mmol})$ in two steps $48 \%$ yield. ${ }^{1} \mathrm{H} \mathrm{NMR}(500 \mathrm{MHz}$, $\left.\mathrm{CDCl}_{3}\right) \delta 9.11(\mathrm{~d}, J=6.6 \mathrm{~Hz}, 1 \mathrm{H}), 7.79(\mathrm{dd}, J=7.9,1.6 \mathrm{~Hz}, 1 \mathrm{H}), 7.47(b r \mathrm{~s}, 1 \mathrm{H}), 7.41-7.32(\mathrm{~m}, 6 \mathrm{H}), 7.28-7.25$ $(\mathrm{m}, 1 \mathrm{H}), 7.21(\mathrm{dd}, J=7.9,1.6 \mathrm{~Hz}, 1 \mathrm{H}), 7.12-7.10(\mathrm{~m}, 1 \mathrm{H}), 6.99(\mathrm{t}, J=7.9 \mathrm{~Hz}, 1 \mathrm{H}), 6.96(b r \mathrm{~d}, J=8.5 \mathrm{~Hz}, 1 \mathrm{H})$, $5.65(\mathrm{~d}, J=12.2 \mathrm{~Hz}, 1 \mathrm{H}), 5.61(\mathrm{~d}, J=12.3 \mathrm{~Hz}, 1 \mathrm{H}), 5.38(\mathrm{~d}, J=13.8 \mathrm{~Hz}, 1 \mathrm{H}), 5.33(\mathrm{~d}, J=13.8 \mathrm{~Hz}, 1 \mathrm{H}), 4.80$ (s, 2H), $4.79-4.74(\mathrm{~m}, 1 \mathrm{H}), 4.52-4.50(\mathrm{~m}, 1 \mathrm{H}), 4.22(\mathrm{dd}, J=9.8,2.1 \mathrm{~Hz}, 1 \mathrm{H}), 4.17(\mathrm{dd}, J=10.4,4.8 \mathrm{~Hz}, 1 \mathrm{H}), 3.75$ $(\mathrm{dd}, J=10.4,7.7 \mathrm{~Hz}, 1 \mathrm{H}), 3.71(\mathrm{dd}, J=9.9,4.3 \mathrm{~Hz}, 1 \mathrm{H}), 3.70-3.63(\mathrm{~m}, 1 \mathrm{H}), 3.57-3.51(\mathrm{~m}, 1 \mathrm{H}), 3.34-3.28(\mathrm{~m}$, $1 \mathrm{H}), 3.23-3.17(\mathrm{~m}, 1 \mathrm{H}), 2.04(\mathrm{~s}, 3 \mathrm{H}), 1.69-1.63(\mathrm{~m}, 2 \mathrm{H}), 1.57-1.47(\mathrm{~m}, 2 \mathrm{H}), 0.80(\mathrm{~s}, 9 \mathrm{H}), 0.02(\mathrm{~s}, 3 \mathrm{H}), 0.00(\mathrm{~s}$, $3 \mathrm{H}) .{ }^{13} \mathrm{C}$ NMR $\left(125 \mathrm{MHz}, \mathrm{CDCl}_{3}\right) \delta 172.9,170.4,170.0,165.2,150.2,149.7,136.5,134.3,133.8,130.5,129.3$, 129.3, 129.2, 128.9, 128.8, 127.8, 126.8, 126.4, 124.6, 123.0, 76.6, 76.6, 75.1, 63.0, 62.9, 55.3, 55.1, 38.9, 26.1, 25.8, 24.1, 20.5, 18.2, 14.3, -5.4, -5.5. HR-MS (ESI-TOF) $m / z$ for $\left[\mathrm{C}_{40} \mathrm{H}_{54} \mathrm{~N}_{4} \mathrm{NaO}_{9} \mathrm{Si}\right]^{+}\left([\mathrm{M}+\mathrm{Na}]^{+}\right)$: calculated 
785.3558, found 785.3555 .

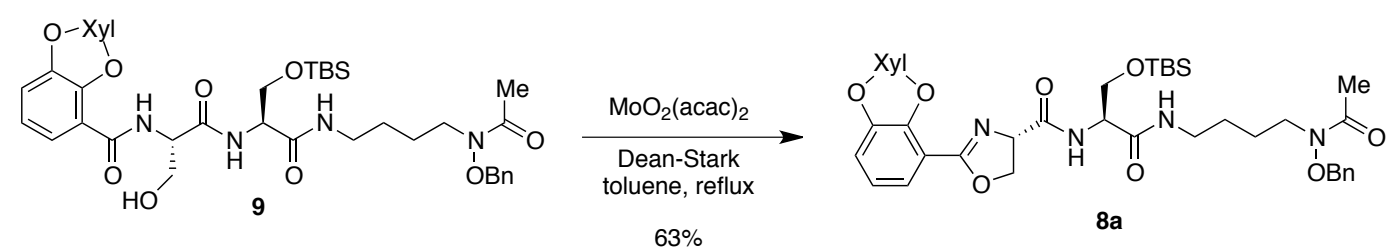

Compound 8a (stereoselective condition). The reaction was carried out in a flask fitted with a pressure-equalized addition funnel (containing a cotton plug and molecular sieves to function as a Soxhlet extractor) surmounted with a reflux condenser. A solution of $9(0.882 \mathrm{~g}, 1.16 \mathrm{mmol})$ and $\mathrm{MoO}_{2}(\mathrm{acac})_{2}(75 \mathrm{mg}, 20 \mathrm{~mol} \%)$ in toluene $(230 \mathrm{~mL})$ was heated with an oil bath under azeotropic reflux. After stirred for $8 \mathrm{~h}$, the reaction mixture was cooled to room temperature and concentrated under reduced pressure. The crude residue was then subjected to flash column chromatography $\left(\mathrm{SiO}_{2}, 2 \%\right.$ methanol in dichloromethane) to afford the desired amide $\mathbf{8 a}(0.545 \mathrm{~g}, 0.730 \mathrm{mmol})$ in $63 \%$ yield without formation of its diastereomer $\mathbf{8 b}$.

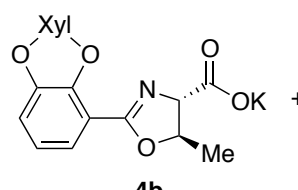

4b

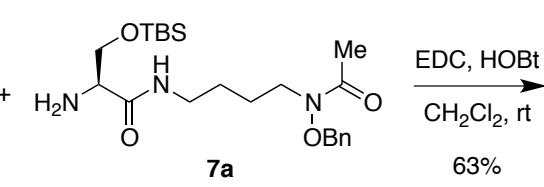

$7 a$

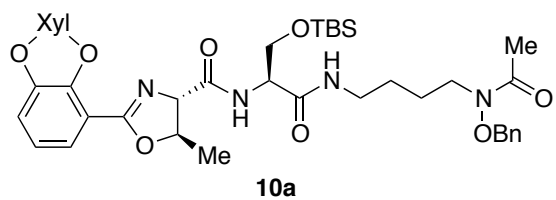

Compound 10a. The synthesis of compound 10a from $4 \mathbf{b}$ and $7 \mathbf{a}$ was previously reported in Ref. S2.
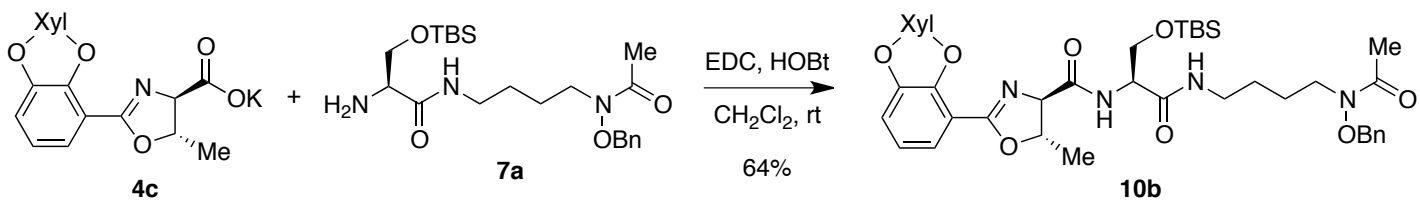

Compound 10b. To a solution of amine $7 \mathbf{a}(0.220 \mathrm{~g}, 0.502 \mathrm{mmol})$ and potassium carboxylate $4 \mathbf{c}(0.236 \mathrm{~g}, 0.625$ $\mathrm{mmol})$ in dichloromethane $(3 \mathrm{~mL})$ was added EDC $(0.120 \mathrm{~g}, 0.781 \mathrm{mmol})$ and $\mathrm{HOBt}(0.140 \mathrm{~g}, 0.781 \mathrm{mmol})$ at 0 ${ }^{\circ} \mathrm{C}$. The reaction mixture was stirred at room temperature for $5 \mathrm{hr}$, and then it was diluted with dichloromethane (25 $\mathrm{mL}$ ) and $1 \mathrm{~N} \mathrm{HCl}$ solution $(25 \mathrm{~mL})$. After collection of the organic layer, the aqueous layer was extracted with dichloromethane $(20 \mathrm{~mL} \times 2)$. The combined organic layers were then washed with saturated sodium bicarbonate solution and brine, dried over anhydrous magnesium sulfate, and concentrated under reduced pressure. The crude residue was subjected to flash column chromatography $\left(\mathrm{SiO}_{2}, 3 \%\right.$ methanol in dichloromethane) to afford the desired amide $10 b(244 \mathrm{mg}, 0.322 \mathrm{mmol})$ in $64 \%$ yield. ${ }^{1} \mathrm{H}$ NMR $\left(500 \mathrm{MHz}, \mathrm{CDCl}_{3}\right) \delta 7.65(\mathrm{~d}, J=6.7 \mathrm{~Hz}, 1 \mathrm{H})$, $7.44(\mathrm{dd}, J=7.8,1.7 \mathrm{~Hz}, 1 \mathrm{H}), 7.39-7.36(\mathrm{~m}, 3 \mathrm{H}), 7.35-7.31(\mathrm{~m}, 2 \mathrm{H}), 7.27-7.25(\mathrm{~m}, 1 \mathrm{H}), 7.22-7.18(\mathrm{~m}, 1 \mathrm{H})$, $7.13(\mathrm{dd}, J=8.0,1.7 \mathrm{~Hz}, 1 \mathrm{H}), 6.95(\mathrm{t}, J=7.9 \mathrm{~Hz}, 1 \mathrm{H}), 6.57(b r \mathrm{t}, J=5.5 \mathrm{~Hz}, 1 \mathrm{H}), 5.64(\mathrm{~d}, J=13.5 \mathrm{~Hz}, 1 \mathrm{H}), 5.50$ $(\mathrm{d}, J=11.4 \mathrm{~Hz}, 1 \mathrm{H}), 5.47(\mathrm{~d}, J=11.9 \mathrm{~Hz}, 1 \mathrm{H}), 5.34(\mathrm{~d}, J=13.0 \mathrm{~Hz}, 1 \mathrm{H}), 4.89-4.83(\mathrm{~m}, 1 \mathrm{H}), 4.76(\mathrm{~s}, 2 \mathrm{H}),, 4.38$ 
- $4.34(\mathrm{~m}, 1 \mathrm{H}), 4.06(\mathrm{dd}, J=9.7,4.2 \mathrm{~Hz}, 1 \mathrm{H}), 3.63-3.58(\mathrm{~m}, 2 \mathrm{H}), 3.27$ (q, $J=6.9 \mathrm{~Hz}, 2 \mathrm{H}), 2.05$ (s, $3 \mathrm{H}), 1.68-$ $1.58(\mathrm{~m}, 2 \mathrm{H}), 1.59(\mathrm{~d}, J=6.2 \mathrm{~Hz}, 3 \mathrm{H}), 1.51-1.45(\mathrm{~m}, 2 \mathrm{H}), 0.88(\mathrm{~s}, 9 \mathrm{H}), 0.11(\mathrm{~s}, 3 \mathrm{H}), 0.08(\mathrm{~s}, 3 \mathrm{H}) .{ }^{13} \mathrm{C}$ NMR $(125$ $\left.\mathrm{MHz}, \mathrm{CDCl}_{3}\right) \delta 172.3,171.8,169.8,163.9,151.5,149.9,136.0,135.4,134.4,129.2,129.01,128.97,128.9,128.8$, $128.6,128.4,125.0,123.3,121.6,78.9,76.3,76.0,75.9,75.1,62.7,53.9,44.8,39.2,26.7,25.8,24.4,22.0,20.5$, 18.1, -5.41, -5.49. HR-MS (ESI-TOF) $\mathrm{m} / z$ for $\left[\mathrm{C}_{41} \mathrm{H}_{55} \mathrm{~N}_{4} \mathrm{O}_{8} \mathrm{Si}\right]^{+}\left([\mathrm{M}+\mathrm{H}]^{+}\right)$: calculated 759.3789 , found 759.3787 .

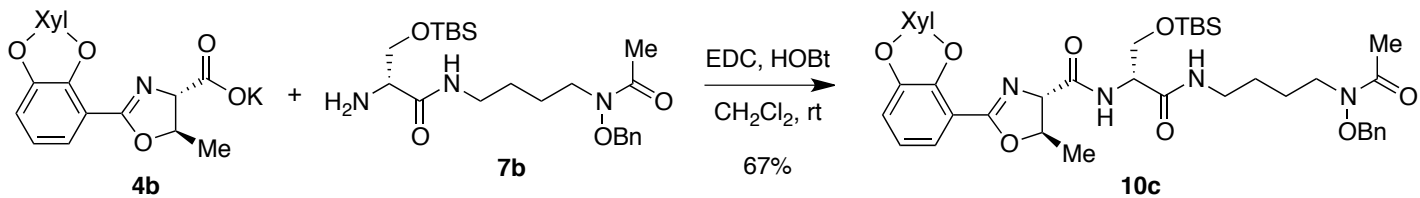

Compound 10c. Compound 10c was synthesized analogously to preparation of compound $\mathbf{1 0 b}$, in which the reaction between amine $7 \mathbf{b}(0.476 \mathrm{~g}, 0.721 \mathrm{mmol})$ and potassium carboxylate $4 \mathbf{b}(0.326 \mathrm{~g}, 1.87 \mathrm{mmol})$ led to formation of the desired product $10 \mathrm{c}(0.360 \mathrm{~g}, 0.483 \mathrm{mmol})$ in $67 \%$ yield. The ${ }^{1} \mathrm{H}-,{ }^{13} \mathrm{C}-\mathrm{NMR}$, and HR-MS spectra of compound $\mathbf{1 0 c}$ were were identical to those of its enantiomer, compound $\mathbf{1 0 b}$.

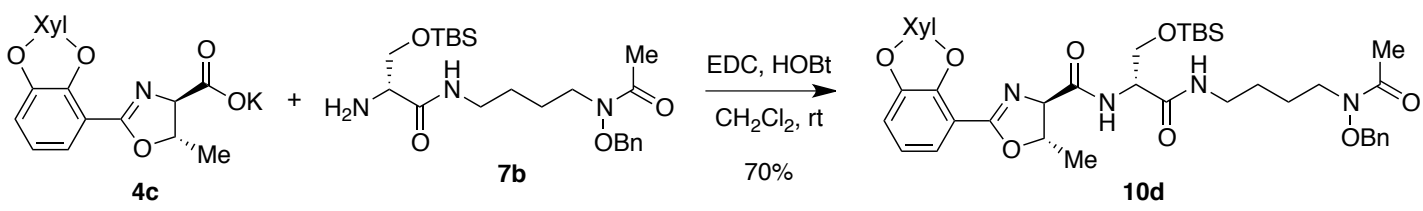

Compound 10d. Compound 10d was synthesized analogously to preparation of compound 10b, in which the reaction between amine $7 \mathbf{b}(1.07 \mathrm{~g}, 2.45 \mathrm{mmol})$ and potassium carboxylate $4 \mathbf{c}(1.01 \mathrm{~g}, 2.70 \mathrm{mmol})$ led to formation of the desired product $10 \mathrm{~d}(1.30 \mathrm{~g}, 1.72 \mathrm{mmol})$ in $70 \%$ yield. The ${ }^{1} \mathrm{H}-,{ }^{13} \mathrm{C}-\mathrm{NMR}$, and HR-MS spectra of compound 10d were identical to those of its enantiomer, compound 10a.

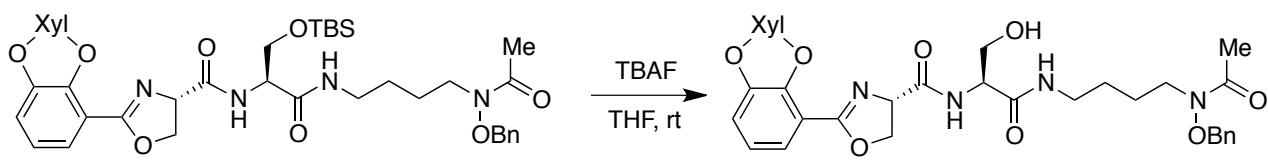

$8 \mathbf{a}$

1a-i

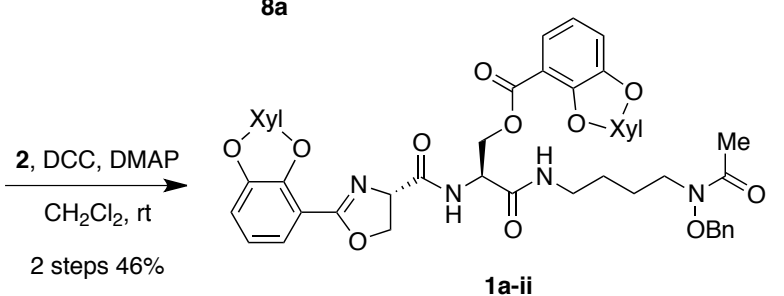

Compound 1a-ii. To a solution of $8 \mathbf{a}(0.248 \mathrm{~g}, 0.323 \mathrm{mmol})$ in tetrahydrofuran (THF, $2 \mathrm{~mL})$ was added tetrabutylammonium fluoride (TBAF, $1 \mathrm{M}$ in THF, $0.485 \mathrm{~mL}, 0.485 \mathrm{mmol}$ ) at $0{ }^{\circ} \mathrm{C}$, and the resulting mixture was stirred at room temperature for $5 \mathrm{hr}$. After the substrate was completely consumed, the solvent was removed under reduced pressure. The resulting crude product 1a-i was used in the next step without further purification. To a 
solution of crude 1a-i and acid 2 (0.165 g, $0.646 \mathrm{mmol})$ in dichloromethane $(2 \mathrm{~mL})$ was added dicyclohexylcarbodiimide (DCC, $0.199 \mathrm{~g}, 0.969 \mathrm{mmol}$ ) and 4-(dimethylamino)pyridine (DMAP, $11.8 \mathrm{mg}, 0.0969$ $\mathrm{mmol})$ at room temperature. After stirred for $3 \mathrm{hr}$, the reaction mixture was diluted with dichloromethane $(10 \mathrm{~mL})$ and $1 \mathrm{~N}$ hydrochloric acid solution $(10 \mathrm{~mL})$. After collecting the organic layer, the aqueous layer was extracted with dichloromethane $(2 \times 20 \mathrm{~mL})$. The combined organic layers were washed with saturated sodium bicarbonate solution and brine, dried over anhydrous magnesium sulfate, and concentrated under the reduced pressure. The crude residue was purified by flash column chromatography $\left(\mathrm{SiO}_{2}, 3 \%\right.$ methanol in dichloromethane) to afford the ester intermediate 1a-ii $(0.129 \mathrm{~g}, 0.149 \mathrm{mmol})$ in a two-step $46 \%$ yield. ${ }^{1} \mathrm{H}$ NMR $\left(500 \mathrm{MHz}\right.$, methanol- $\left.d^{4}\right) \delta 7.38$ $(\mathrm{dd}, \mathrm{J}=7.8,1.7 \mathrm{~Hz}, 1 \mathrm{H}), 7.37-7.33(\mathrm{~m}, 6 \mathrm{H}), 7.26-7.19(\mathrm{~m}, 8 \mathrm{H}), 7.16-7.11(\operatorname{app} \mathrm{t}, J=1.5 \mathrm{~Hz}, 1 \mathrm{H}), 7.12$ (app t, $J=1.5 \mathrm{~Hz}, 1 \mathrm{H}), 6.94(\mathrm{t}, J=7.9 \mathrm{~Hz}, 1 \mathrm{H}), 6.88(\mathrm{t}, J=7.9 \mathrm{~Hz}, 1 \mathrm{H}), 5.48(\mathrm{~d}, J=12.9 \mathrm{~Hz}, 1 \mathrm{H}), 5.45(\mathrm{~s}, 2 \mathrm{H}), 5.42(\mathrm{~d}, J$ $=12.9 \mathrm{~Hz}, 1 \mathrm{H}), 5.38(\mathrm{~d}, J=12.9 \mathrm{~Hz}, 1 \mathrm{H}), 5.35(\mathrm{~s}, 2 \mathrm{H}), 5.34(\mathrm{~d}, J=12.9 \mathrm{~Hz}, 1 \mathrm{H}), 4.95(\mathrm{dd}, J=10.7,8.1 \mathrm{~Hz}, 1 \mathrm{H})$, $4.82(\mathrm{t}, J=4.8 \mathrm{~Hz}, 1 \mathrm{H}), 4.78(\mathrm{~s}, 2 \mathrm{H}), 4.64(\mathrm{dd}, J=4.8,2.1 \mathrm{~Hz}, 2 \mathrm{H}), 4.60-4.52(\mathrm{~m}, 2 \mathrm{H}), 3.58(b r \mathrm{~m}, 2 \mathrm{H}), 3.29-$ $3.24(\mathrm{~m}, 1 \mathrm{H}), 3.21-3.15(\mathrm{~m}, 1 \mathrm{H}), 1.95(\mathrm{~s}, 3 \mathrm{H}), 1.61-1.55(\mathrm{~m}, 2 \mathrm{H}), 1.49-1.43(\mathrm{~m}, 2 \mathrm{H}) .{ }^{13} \mathrm{C} \mathrm{NMR}(125 \mathrm{MHz}$ methanol- $\left.d^{4}\right) \delta 173.9,170.3,166.9,166.6,152.5,152.4,151.1,150.9,137.0,137.0,136.92,136.89,136.0,130.7$, $130.3,130.2$, 130.1 130.0, 129.9, 129.8, 129.73, 129.71, 129.69, 127.1, 126.5, 126.5, 126.2, 125.7, 124.2, 124.2, $122.1,77.1,76.6,76.3,76.1,75.8,70.8,70.4,65.1,54.8,53.9,40.1,29.5,27.5,20.4$. HR-MS (ESI-TOF) $m / z$ for $\left[\mathrm{C}_{49} \mathrm{H}_{49} \mathrm{~N}_{4} \mathrm{O}_{11}\right]^{+}\left([\mathrm{M}+\mathrm{H}]^{+}\right)$: calculated 869.3398, found 869.3395 .

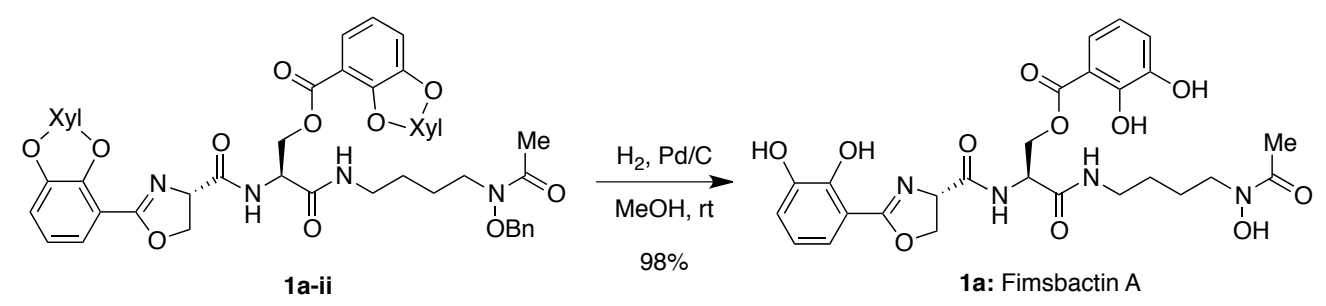

Fimsbactin A (1a). For the hydrogenolytic global deprotection, a catalytic amount of palladium on activated carbon (10 wt\%, $15.9 \mathrm{mg}, 0.015 \mathrm{mmol})$ was suspended in a solution of ester 1a-ii (26.3 mg, $0.0303 \mathrm{mmol})$ in methanol (1 $\mathrm{mL}$ ) at room temperature, and the reaction was initiated by charging the reaction flask with hydrogen gas in a balloon (1 atm). After $3 \mathrm{hr}$, the reaction mixture was filtered through a pad of celite to remove the palladium catalyst, and the filtrate was concentrated under reduced pressure to afford the desired product 1a (Fimsbactin A, $17.7 \mathrm{mg}$, $0.0297 \mathrm{mmol})$ in $98 \%$ yield without further purification. ${ }^{1} \mathrm{H}$ NMR $\left(500 \mathrm{MHz}, \mathrm{DMSO}-d^{6}\right) \delta 8.80(b r \mathrm{~s}, 1 \mathrm{H}), 8.31(b r$ $\mathrm{s}, 1 \mathrm{H}), 7.14(\mathrm{~d}, J=7.7 \mathrm{~Hz}, 1 \mathrm{H}), 7.09(\mathrm{~d}, J=7.7 \mathrm{~Hz}, 1 \mathrm{H}), 6.96($ app t, $J=8.0 \mathrm{~Hz}, 2 \mathrm{H}), 6.74($ app t, $J=7.6 \mathrm{~Hz}, 1 \mathrm{H})$, 6.55 (app t, $J=7.6 \mathrm{~Hz}, 1 \mathrm{H}), 5.07(\mathrm{dd}, J=10.0,7.5 \mathrm{~Hz}, 1 \mathrm{H}), 4.73-4.68(\mathrm{~m}, 1 \mathrm{H}), 4.63-4.58(b r \mathrm{~m}, 1 \mathrm{H}), 4.57$ (dd, $J$ $=11,4.7 \mathrm{~Hz}, 1 \mathrm{H}), 4.52(\operatorname{app~t}, J=7.7 \mathrm{~Hz}, 1 \mathrm{H}), 4.42-4.37(\mathrm{~m}, 1 \mathrm{H}), 3.47-3.43(\mathrm{~m}, 2 \mathrm{H}), 3.13-3.07(\mathrm{~m}, 2 \mathrm{H}), 1.96(\mathrm{~s}$, $3 \mathrm{H}), 1.53-1.46(\mathrm{~m}, 2 \mathrm{H}), 1.43-1.35(\mathrm{~m}, 2 \mathrm{H}) .{ }^{13} \mathrm{C}$ NMR (125 MHz, DMSO- $\left.d^{6}\right) \delta 170.2,170.1,168.9,167.9,166.5$, 
148.3, 146.2, 145.8, 120.4, 119.8, 119.5, 118.7, 117.9, 112.7, 110.1, 69.3, 67.2, 64.4, 51.7, 46.5, 38.5, 26.1, 23.7, 20.4. HR-MS (ESI-TOF) $m / z$ for $\left[\mathrm{C}_{26} \mathrm{H}_{30} \mathrm{~N}_{4} \mathrm{NaO}_{11}\right]^{+}\left([\mathrm{M}+\mathrm{Na}]^{+}\right)$: calculated 597.1809, found 597.1807.

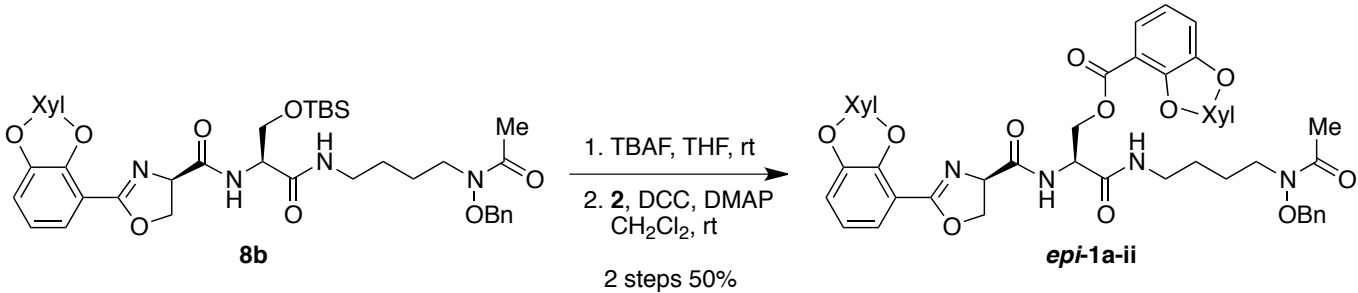

Compound epi-1a-ii. Compound epi-1a-ii was synthesized analogously to preparation of compound 1a-ii, in which a two-reaction sequence from $\mathbf{8 b}(30 \mathrm{mg}, 0.035 \mathrm{mmol})$ led to formation of the desired product epi-1a-ii $(15.3 \mathrm{mg}$, $0.0175 \mathrm{mmol})$ in two step 50\% yield. ${ }^{1} \mathrm{H}$ NMR (500 MHz, methanol- $\left.d^{4}\right) \delta 7.39-7.32(\mathrm{~m}, 5 \mathrm{H}), 7.24-7.12(\mathrm{~m}$, 10H), $7.07(\mathrm{dd}, J=8.1,1.7 \mathrm{~Hz}, 1 \mathrm{H}), 7.00(\mathrm{dd}, J=8.0,1.7 \mathrm{~Hz}, 1 \mathrm{H}), 6.81(\mathrm{t}, J=7.9 \mathrm{~Hz}, 1 \mathrm{H}), 6.64(\mathrm{t}, J=7.9 \mathrm{~Hz}$, $1 \mathrm{H}), 5.44(\mathrm{~d}, J=13.1 \mathrm{~Hz}, 1 \mathrm{H}), 5.36(\mathrm{~d}, J=13.0 \mathrm{~Hz}, 1 \mathrm{H}), 5.33(\mathrm{~s}, 2 \mathrm{H}), 5.32(\mathrm{~d}, J=12.9 \mathrm{~Hz}, 1 \mathrm{H}), 5.28(\mathrm{~s} 1 \mathrm{H}), 5.26$ $(\mathrm{s}, 1 \mathrm{H}), 5.23(\mathrm{~d}, J=12.9 \mathrm{~Hz}, 1 \mathrm{H}), 4.94(\mathrm{dd}, J=10.9,7.8 \mathrm{~Hz}, 1 \mathrm{H}), 4.81(\mathrm{~s}, 2 \mathrm{H}), 4.72(\mathrm{dd}, J=11.4,4.0 \mathrm{~Hz}, 1 \mathrm{H}), 4.60$

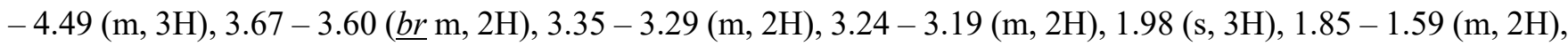
$1.53-1.47(\mathrm{~m}, 2 \mathrm{H}) .{ }^{13} \mathrm{C}$ NMR $\left(125 \mathrm{MHz}\right.$, methanol- $\left.d^{4}\right) \delta 173.8,170.3,167.0,166.5,152.6,152.5,151.3,150.9$, 137.1, 136.99, 136.95, 130.7, 130.30, 130.28, 130.1, 129.98, 129.97, 129.82, 129.79, 129.76, 129.7, 127.3, 126.6, 126.5, 126.2, 125.8, 124.4, 124.3, 122.3, 77.10, 77.05, 76.5, 76.3, 76.0, 70.7, 70.4, 65.4, 54.8, 53.8, 40.1, 34.6, 27.5, 20.3. HR-MS (ESI-TOF) $m / z$ for $\left[\mathrm{C}_{49} \mathrm{H}_{49} \mathrm{~N}_{4} \mathrm{O}_{11}\right]^{+}\left([\mathrm{M}+\mathrm{H}]^{+}\right)$: calculated 869.3398, found 869.3396.

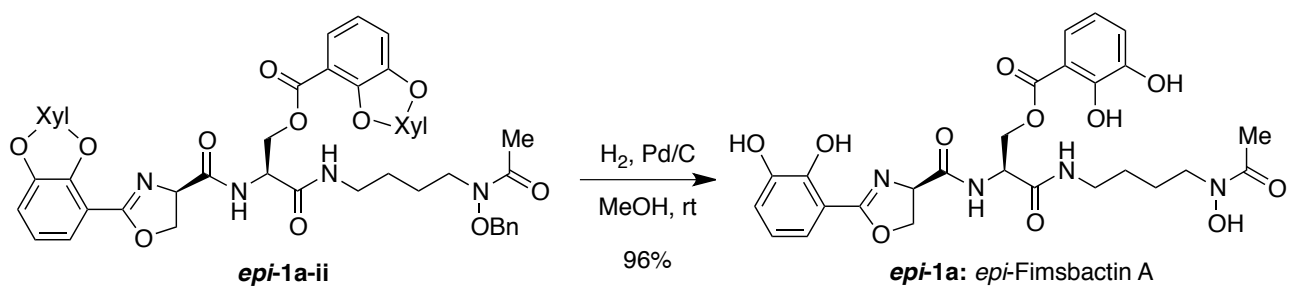

epi-Fimsbactin A (epi-1a). epi-Fimsbactin A (epi-1a) was synthesized analogously to preparation of fimsbactin A (1a), in which epi-1a-ii (15.3 mg, $0.0175 \mathrm{mmol})$ was subjected to hydrogenolysis to afford epi-fimsbactin A (epi1a, $9.3 \mathrm{mg}, 0.016 \mathrm{mmol})$ in 96\% yield. ${ }^{1} \mathrm{H}$ NMR (500 MHz, DMSO- $\left.d^{6}\right) \delta 8.74(b r \mathrm{~s}, 1 \mathrm{H}), 8.39(b r \mathrm{~s}, 1 \mathrm{H}), 7.18(\mathrm{~d}$, $J=7.9 \mathrm{~Hz}, 1 \mathrm{H}), 7.08(\mathrm{~d}, J=7.8 \mathrm{~Hz}, 1 \mathrm{H}), 6.97(b r \mathrm{~d}, J=7.4 \mathrm{~Hz}, 2 \mathrm{H}), 6.74(\operatorname{app} \mathrm{t}, J=7.8 \mathrm{~Hz}, 1 \mathrm{H}), 6.64(\operatorname{app} \mathrm{t}, J=$ $7.8 \mathrm{~Hz}, 1 \mathrm{H}), 5.07$ (dd, $J=10.0,7.5 \mathrm{~Hz}, 1 \mathrm{H}), 4.76-4.71(\mathrm{~m}, 1 \mathrm{H}), 4.63-4.57(b r \mathrm{~m}, 1 \mathrm{H}), 4.49-4.42(\mathrm{~m}, 3 \mathrm{H}), 3.44(\mathrm{t}$, $J=6.5 \mathrm{~Hz}, 2 \mathrm{H}), 3.16-3.02(\mathrm{~m}, 2 \mathrm{H}), 1.95(\mathrm{~s}, 3 \mathrm{H}), 1.52-1.44(\mathrm{~m}, 2 \mathrm{H}), 1.45-1.32(\mathrm{~m}, 2 \mathrm{H}) .{ }^{13} \mathrm{C} \mathrm{NMR}(125 \mathrm{MHz}$, DMSO- $\left.d^{6}\right) \delta 170.2,170.0,168.8,168.0,166.6,148.3,146.8,146.4,145.8,119.9,119.4,118.8,118.7,117.9,112.6$, 110.1, 69.4, 67.1, 64.2, 51.6, 46.4, 38.5, 26.0, 23.7, 20.4. HR-MS (ESI-TOF) $m / z$ for $\left[\mathrm{C}_{26} \mathrm{H}_{30} \mathrm{~N}_{4} \mathrm{NaO}_{11}\right]^{+}\left([\mathrm{M}+\mathrm{Na}]^{+}\right)$: calculated 597.1809, found 597.1806. 


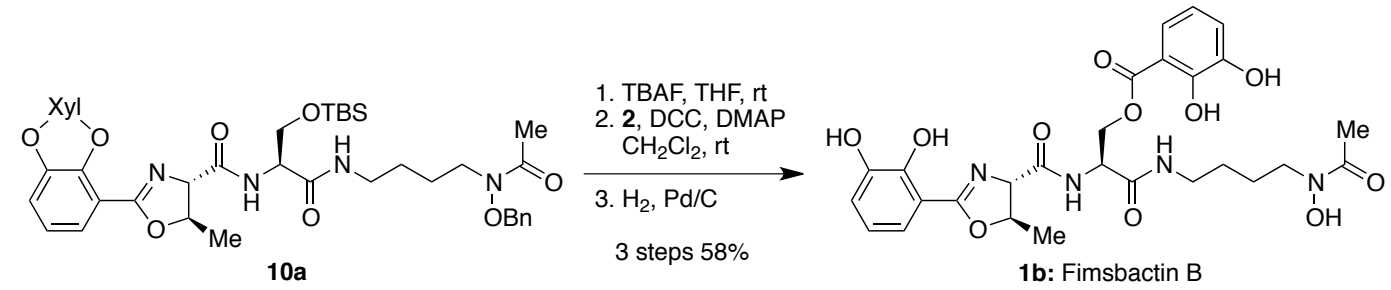

Fimsbactin B (1b). Compound $\mathbf{1 b}$ was synthesized analogously to preparation of compound $\mathbf{1 a}$, in which a threereaction sequence from $\mathbf{1 0 a}(0.291 \mathrm{~g}, 0.391 \mathrm{mmol})$ led to formation of the desired product $\mathbf{1 b}(0.133 \mathrm{~g}, 0.227 \mathrm{mmol})$ in three step $58 \%$ yield. The ${ }^{1} \mathrm{H}-,{ }^{13} \mathrm{C}-\mathrm{NMR}$, and HR-MS spectra of compound $\mathbf{1 b}$ were previously reported in Ref. S2.

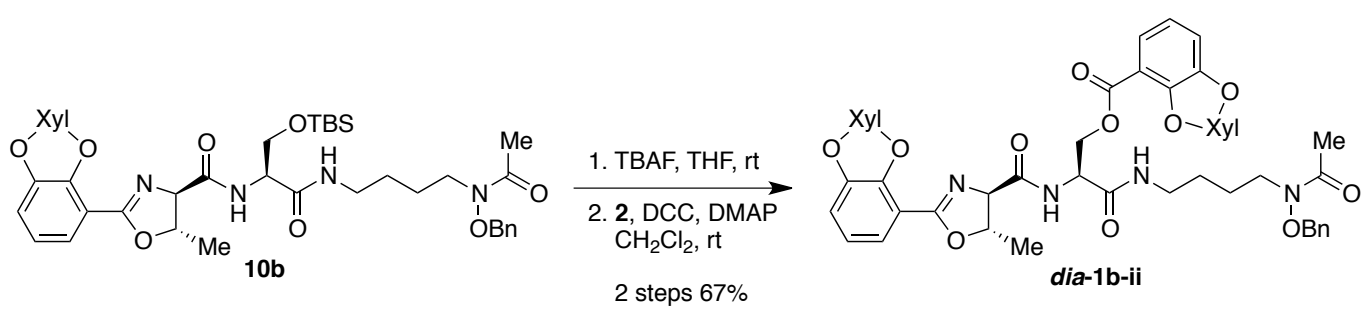

Compound dia-1b-ii. Compound dia-1b-ii was synthesized analogously to preparation of compound 1a-ii, in which a two-reaction sequence from $10 b(0.092 \mathrm{~g}, 0.140 \mathrm{mmol})$ led to formation of the desired product dia-1b-ii $(63.3 \mathrm{mg}, 0.094 \mathrm{mmol})$ in two step $67 \%$ yield. ${ }^{1} \mathrm{H}$ NMR $\left(500 \mathrm{MHz}, \mathrm{CDCl}_{3}\right) \delta 7.75(\mathrm{~d}, J=7.4 \mathrm{~Hz}, 1 \mathrm{H}), 7.44(\mathrm{dd}, J$ $=7.8,1.6 \mathrm{~Hz}, 1 \mathrm{H}), 7.42(\mathrm{dd}, J=7.8,1.6 \mathrm{~Hz}, 1 \mathrm{H}), 7.38-7.33(\mathrm{~m}, 3 \mathrm{H}), 7.32-7.28(\mathrm{~m}, 2 \mathrm{H}), 7.27-7.13(\mathrm{~m}, 9 \mathrm{H})$, $7.11(\mathrm{dd}, J=8.0,1.6 \mathrm{~Hz}, 1 \mathrm{H}), 6.93(\mathrm{t}, J=7.9 \mathrm{~Hz}, 1 \mathrm{H}), 6.91(\mathrm{t}, J=7.9 \mathrm{~Hz}, 1 \mathrm{H}), 6.84(b r \mathrm{t}, J=5.3 \mathrm{~Hz}, 1 \mathrm{H}), 5.58(\mathrm{~d}$, $J=13.4 \mathrm{~Hz}, 1 \mathrm{H}), 5.49-5.42(\mathrm{~m}, 4 \mathrm{H}), 5.37-5.29(\mathrm{~m}, 3 \mathrm{H}), 4.90-4.85(\mathrm{~m}, 1 \mathrm{H}), 4.83-4.79(\mathrm{~m}, 1 \mathrm{H}), 4.74-4.71$ $(\mathrm{m}, 1 \mathrm{H}), 4.71(\mathrm{~s}, 2 \mathrm{H}), 3.51(b r \mathrm{t}, J=7.2 \mathrm{~Hz}, 2 \mathrm{H}), 3.25-3.18(\mathrm{~m}, 1 \mathrm{H}), 3.15-3.09(\mathrm{~m}, 1 \mathrm{H}), 2.01(\mathrm{~s}, 3 \mathrm{H}), 1.56(\mathrm{~d}, J$ $=6.2 \mathrm{~Hz}, 3 \mathrm{H}), 1.54-1.48(\mathrm{~m}, 2 \mathrm{H}), 1.38-1.32(\mathrm{~m}, 2 \mathrm{H}) .{ }^{13} \mathrm{C} \mathrm{NMR}\left(125 \mathrm{MHz}, \mathrm{CDCl}_{3}\right) \delta 172.1,168.5,165.5,163.9$, $151.6,151.5,150.3,150.0,136.0,135.6,135.5,135.4,129.3,129.13,129.06,129.0,128.9,128.9,128.8,128.7$, $128.5,126.3,125.9,125.1,125.1,124.4,123.4,121.6,78.8,76.4,76.0,75.9,75.4,75.2,64.1,52.0,39.5,26.2,24.5$, 22.0, 20.5. HR-MS (ESI-TOF) $m / z$ for $\left[\mathrm{C}_{50} \mathrm{H}_{51} \mathrm{~N}_{4} \mathrm{O}_{11}\right]^{+}\left([\mathrm{M}+\mathrm{H}]^{+}\right)$: calculated 883.3554, found 883.3552. 


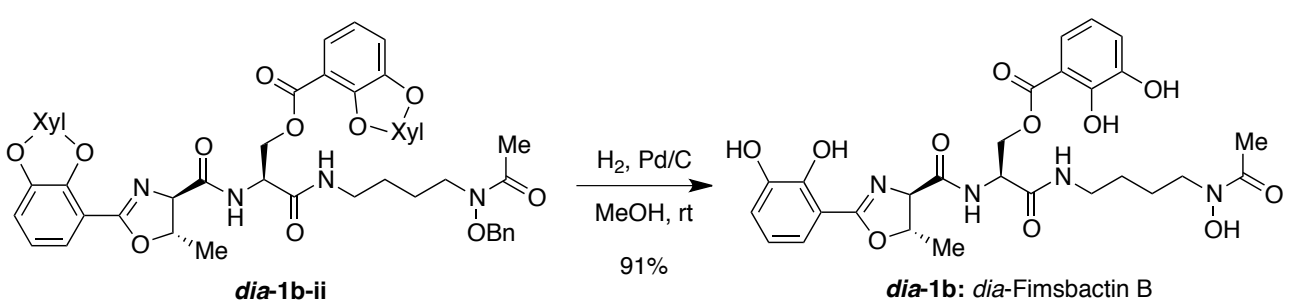

dia-Fimsbactin B (dia-1b). dia-Fimsbactin B (dia-1b, $50.9 \mathrm{mg}, 0.086 \mathrm{mmol})$ was synthesized from dia-1b-ii (63.3 $\mathrm{mg}, 0.094 \mathrm{mmol}$ ) by hydrogenolytic global deprotection, analogously to preparation of fimsbactin A (1a), and the yield was 91\%. ${ }^{1} \mathrm{H}$ NMR (500 MHz, DMSO- $\left.d^{6}\right) \delta 11.81(\mathrm{~s}, 1 \mathrm{H}), 10.26(\mathrm{~s}, 1 \mathrm{H}), 9.66(\mathrm{~s}, 1 \mathrm{H}), 9.45$ (s, $\left.1 \mathrm{H}\right), 9.26(\mathrm{~s}$, $1 \mathrm{H}), 8.64(\mathrm{~d}, J=8.2 \mathrm{~Hz}, 1 \mathrm{H}), 8.29(\mathrm{t}, J=5.5 \mathrm{~Hz}, 1 \mathrm{H}), 7.22(\mathrm{dd}, J=8.0,1.5 \mathrm{~Hz}, 1 \mathrm{H}), 7.06(\mathrm{dd}, J=7.9,1.5 \mathrm{~Hz}, 1 \mathrm{H})$, $7.04(\mathrm{dd}, J=7.8,1.5 \mathrm{~Hz}, 1 \mathrm{H}), 6.97(\mathrm{dd}, J=7.9,1.5 \mathrm{~Hz}, 1 \mathrm{H}), 6.75(\operatorname{app} \mathrm{q}, J=8.0 \mathrm{~Hz}, 2 \mathrm{H}), 4.82-4.74(\mathrm{~m}, 2 \mathrm{H})$, $4.60(\mathrm{~d}, J=7.0 \mathrm{~Hz}, 1 \mathrm{H}), 4.54(\mathrm{dd}, J=10.9,5.1 \mathrm{~Hz}, 1 \mathrm{H}), 4.46(\mathrm{dd}, J=10.9,6.7 \mathrm{~Hz}, 1 \mathrm{H}), 3.43(\mathrm{t}, J=6.7 \mathrm{~Hz}, 2 \mathrm{H})$, $3.13(\mathrm{dq}, J=12.6,6.6 \mathrm{~Hz}, 1 \mathrm{H}), 3.05(\mathrm{dq}, J=12.5,6.6 \mathrm{~Hz}, 1 \mathrm{H}), 1.95(\mathrm{~s}, 3 \mathrm{H}), 1.52-1.43(\mathrm{~m}, 2 \mathrm{H}), 1.41$ (d, $J=6.3$ $\mathrm{Hz}, 3 \mathrm{H}), 1.39-1.33(\mathrm{~m}, 2 \mathrm{H}) .{ }^{13} \mathrm{C}$ NMR $\left(125 \mathrm{MHz}, \mathrm{DMSO}-d^{6}\right) \delta 170.2,169.8,168.9,167.8,166.0,149.5,148.3$, 146.1, 145.8, 120.9, 119.8, 119.5, 118.9, 118.7, 117.9, 112.9, 110.2, 79.0, 73.2, 64.4, 51.5, 46.4, 38.5, 26.1, 23.7, 20.5, 20.3. HR-MS (ESI-TOF) $m / z$ for $\left[\mathrm{C}_{27} \mathrm{H}_{32} \mathrm{~N}_{4} \mathrm{NaO}_{11}\right]^{+}\left([\mathrm{M}+\mathrm{Na}]^{+}\right)$: calculated 611.1965 , found 611.1963 .

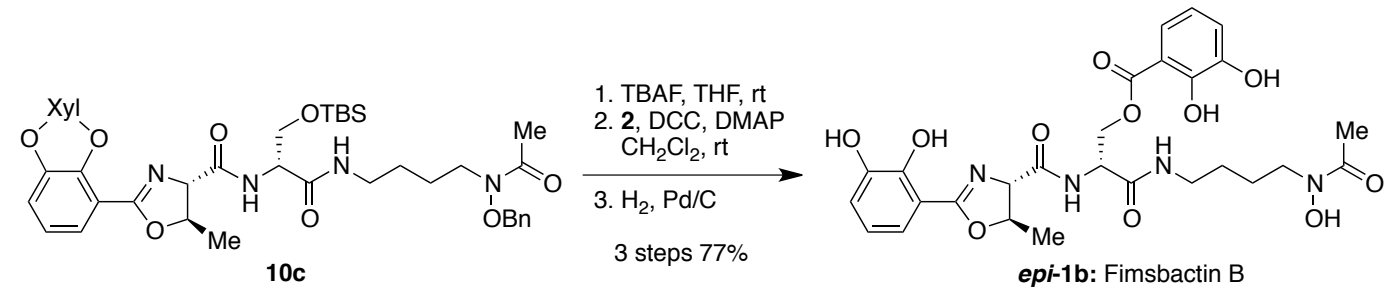

epi-Fimsbactin B (epi-1b). Compound epi-1b was synthesized analogously to preparation of compound 1a, in which a three-reaction sequence from $10 \mathbf{c}(74.3 \mathrm{mg}, 0.1 \mathrm{mmol})$ led to formation of the desired product epi-1b (45.3 mg, $0.077 \mathrm{mmol}$ ) in three step 77\% yield. The ${ }^{1} \mathrm{H}-,{ }^{13} \mathrm{C}-\mathrm{NMR}$, and HR-MS spectra of compound epi-1b were identical to those of its enantiomer, dia-fimsbactin B (dia-1b).

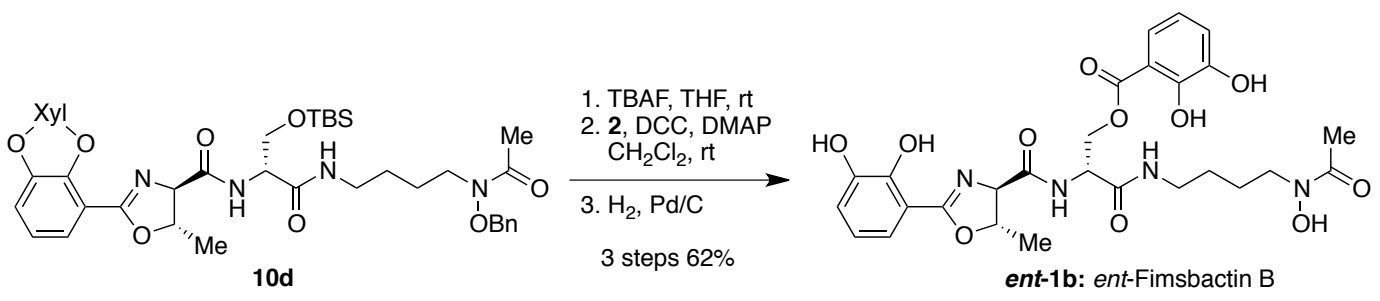

ent-Fimsbactin B (ent-1b). Compound ent-1b was synthesized analogously to preparation of compound 1a, in which a three-reaction sequence from $10 d(0.446 \mathrm{~g}, 0.599 \mathrm{mmol})$ led to formation of the desired product ent-1b $(0.218 \mathrm{~g}, 0.371 \mathrm{mmol})$ in three step $62 \%$ yield. The ${ }^{1} \mathrm{H}-,{ }^{13} \mathrm{C}-\mathrm{NMR}$, and HR-MS spectra of compound ent-1b were identical to those of its enantiomer, fimsbactin B (1b). 


\section{B. Procedures For BiologiCAL/BIOCHEMICAL EXPERIMENTS}

\section{Growth promotion activity assay}

A single colony was picked from a fresh Luria-Bertani (LB) agar-plate overlaid with the A. baumannii strain of interest and was then used to inoculate $5 \mathrm{~mL}$ of LB media. After incubation at $37^{\circ} \mathrm{C}$ overnight in a shaking incubator (200 rpm), the culture solution was diluted with $\mathrm{LB}$ broth for an $\mathrm{OD}_{600} \approx 1$. This solution was further diluted 1:100 with LB media containing the designated concentration of 2,2'-bipyridyl (DP) (275 $\mu \mathrm{M}$ for A. baumannii ATCC 17978, $175 \mu \mathrm{M}$ for A. baumannii ATCC 19606 wild-type or $\Delta$ bauA mutant). An aliquot (198 $\mu \mathrm{L})$ of the diluted culture was mixed with a $2-\mu \mathrm{L}$ aliquot of fimsbactin or fimsbactin stereoisomer DMSO stock solution whose concentrations were adjusted to make the intended final concentrations indicated in Figure 2 in a sterile Greiner Bio-One 96-well microplate (Kremsmünster, Austria). The microplate was then covered with a Breathe-Easy® sealing tape (Sigma-Aldrich) for efficient aeration, and it was incubated at $37{ }^{\circ} \mathrm{C}$ with shaking at $200 \mathrm{rpm}$. The OD600 values were recorded every hour using Epoch 2 microplate reader (Biotek, Vermont, USA). All measurements were made using duplicate or quadruplicate biological samples, and the mean values were used for plotting the results, in which the error bars indicate the standard deviation (Figure 2A and 2B) or the standard error (Figure 2C). A. baumannii ATCC 19606 and 17978 were obtained from ATCC (Virginia, USA) or KCTC (Korean Collection for Type Cultures, Jeollabuk-do, Republic of Korea), and the preparation of A. baumannii ATCC 19606 $\triangle b a u A$ was reported previously. ${ }^{\mathrm{S} 4}$

\section{Determination of the $\mathrm{Fe}$ (III) binding stoichiometry of a fimsbactin}

The fluorescence titration-based determination of the Fe(III) binding stoichiometry of a fimsbactin was conducted by following the procedure described in Ref S5. Briefly, to a $600 \mu \mathrm{L}$ solution of $500 \mu \mathrm{M}$ fimsbactin in ethanol was added $3 \mu \mathrm{L}$ aliquots of $10 \mathrm{mM} \mathrm{FeCl}_{3}$ in ethanol, and emission at $400 \mathrm{~nm}\left(\lambda_{\mathrm{ex}}=320 \mathrm{~nm}\right)$ was recorded after each addition. The emission signal was plotted against the equivalents of Fe(III) as shown in Figure S1. This set of experiments was conducted using a Hitachi F-7000 fluorescence spectrophotometer, in which the slit sizes for excitation and emission were $5 \mathrm{~nm}$ and $10 \mathrm{~nm}$, respectively.

\section{EDTA competition assay}

To qualitatively compare the Fe(III) binding affinity of various fimsbactin isomers, the fluorescence recovery of the corresponding Fe(III) complexes upon addition of a competing Fe(III) chelator, ethylenediaminetetraacetic acid (EDTA), was monitored. The Fe(III)-fimsbactin complex was prepared by dissolving $30 \mu \mathrm{L}$ of $10 \mathrm{mM}$ fimsbactin and $30 \mu \mathrm{L}$ of $10 \mathrm{mM} \mathrm{FeCl}_{3}$ in $540 \mu \mathrm{L}$ ethanol, in which the final concentration of the resulting $\mathrm{Fe}(\mathrm{III})$-fimsbactin complex was $500 \mu \mathrm{M}$. To this solution, aliquots of $10 \mathrm{mM} \mathrm{Na} 4$ EDTA in water were added one after the other to adjust the equivalents of EDTA with respect to Fe(III)-fimsbactin complex to be $0.5,0.75,1,1.25,1.5,1.75$, and 2. 
The fluorescence emission at $400 \mathrm{~nm}\left(\lambda_{\mathrm{ex}}=320 \mathrm{~nm}\right)$ was recorded after each addition. The emission signal versus the equivalent of EDTA was plotted in Figure S2. The experimental setup was identical to the binding stoichiometry determination experiment.

\section{Parallel artificial membrane permeability assay (PAMPA)}

A parallel artificial membrane permeability assay was conducted by following the instruction provided in a Corning Gentest Pre-coated PAMPA Plate System (Cat. No. 353015, Corning, New York, USA). Initially, Fe(III)-preloaded fimsbactin A and B (holo-1a and holo-1b) were prepared by mixing each of these compounds with 1.1 eq. iron(III) acetylacetonate, $\mathrm{Fe}(\mathrm{acac})_{3}$, in methanol, and the mixture was incubated at room temperature overnight for complete complexation. Then, methanol was completely removed by using a spinvac, and the resulting residue was triturated and washed with cold ether several times to afford pure holo-fimsbactins. To the wells of a Receiver plate ("donor wells"), $300 \mu \mathrm{L}$ of a solution of holo-fimsbactin A or B $(200 \mu \mathrm{M})$ in $100 \mathrm{mM}$ Tris $\bullet \mathrm{HCl}$ buffer (pH 7) was added, and the wells of a Filter plate ("acceptor wells") were filled with the buffer alone (200 $\mu \mathrm{L})$. Then, these two plates were coupled, and the resulting assembly was incubated at room temperature for $4 \mathrm{hr}$ without agitation. At the end of the incubation, the plates were separated, and $150 \mu \mathrm{L}$ solutions from each well of both plates were aliquoted and transferred to a Greiner UV-star ${ }^{\circledR}$ 96-well microplate. The final concentrations of compounds in both donor and acceptor wells were analyzed by measuring the absorption with Epoch 2 microplate reader (the standard concentration plot for each holo-fimsbactin was created beforehand). Finally, the permeability of each holofimsbactin was calculated using the following formula:

$$
\begin{aligned}
& \text { Permeability }(\mathrm{cm} / \mathrm{s}): P_{\mathrm{e}}=\left\{-\ln \left[1-\mathrm{C}_{\mathrm{A}}(t) / \mathrm{C}_{\mathrm{eq}}\right]\right\} /\left[\mathrm{A} \times\left(1 / \mathrm{V}_{\mathrm{D}}+1 / \mathrm{V}_{\mathrm{A}}\right) \times t\right] \\
& \mathrm{A}=\text { Filter area }\left(0.3 \mathrm{~cm}^{2}\right) \\
& \mathrm{V}_{\mathrm{D}}=\text { Donor well volume }(0.3 \mathrm{~mL}) \\
& \mathrm{V}_{\mathrm{A}}=\text { Acceptor well volume }(0.2 \mathrm{~mL}) \\
& \mathrm{C}_{\mathrm{A}}(t)=\text { compound concentration in acceptor well at time } t \\
& \mathrm{C}_{\mathrm{D}}(t)=\text { compound concentration in donor well at time } t \\
& \mathrm{C}_{\mathrm{eq}}=\left[\mathrm{C}_{\mathrm{D}}(t) \times \mathrm{V}_{\mathrm{D}}+\mathrm{C}_{\mathrm{A}}(t) \times \mathrm{V}_{\mathrm{A}}\right] /\left(\mathrm{V}_{\mathrm{D}}+\mathrm{V}_{\mathrm{A}}\right)
\end{aligned}
$$




\section{SUPPLEMENTAL REFERENCES}

S1. Kim, J.; Lee, J. E.; Ree, H.; Kim, H. J. Bull. Kor. Chem. Soc. 2015, 36, 439.

S2. Ree, H.; Kim, J.; Song, W. Y.; Lee, J. E.; Kim, H. J. Bull. Kor. Chem. Soc. 2015, 36, 1520.

S3. Proschak, A.; Lubuta, P.; Grün, P.; Löhr, F.; Wilharm, G.; De Berardinis, V.; Bode, H. B. Chembiochem 2013, $14,633$.

S4. Oh, M. H.; Lee, J. C.; Kim, J.; Choi, C. H.; Han, K. Appl. Environ. Microbiol. 2015, 81, 3357.

S5. Bohac, T. J.; Fang, L.; Giblin, D. E.; Wencewicz, T. A. ACS Chem. Biol. 2019, 14, 674. 


\section{SPECTRAL DATA}

${ }^{1} \mathrm{H}-\mathrm{NMR}$ of Compound 3a (500 $\left.\mathrm{MHz}, \mathrm{CDCl}_{3}\right)$

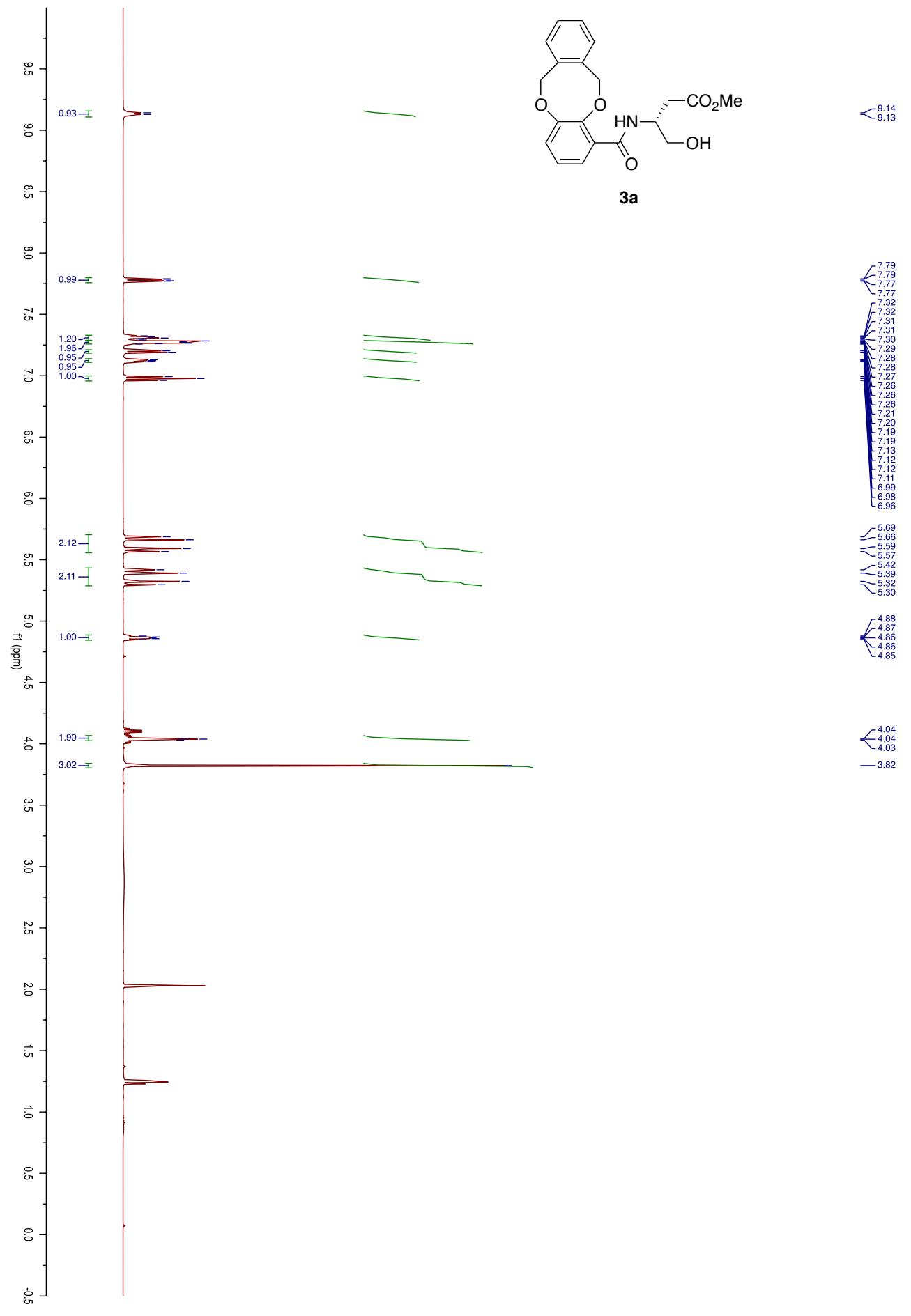




\section{${ }^{13} \mathrm{C}-\mathrm{NMR}$ of Compound 3a (125 $\left.\mathrm{MHz}, \mathrm{CDCl}_{3}\right)$}

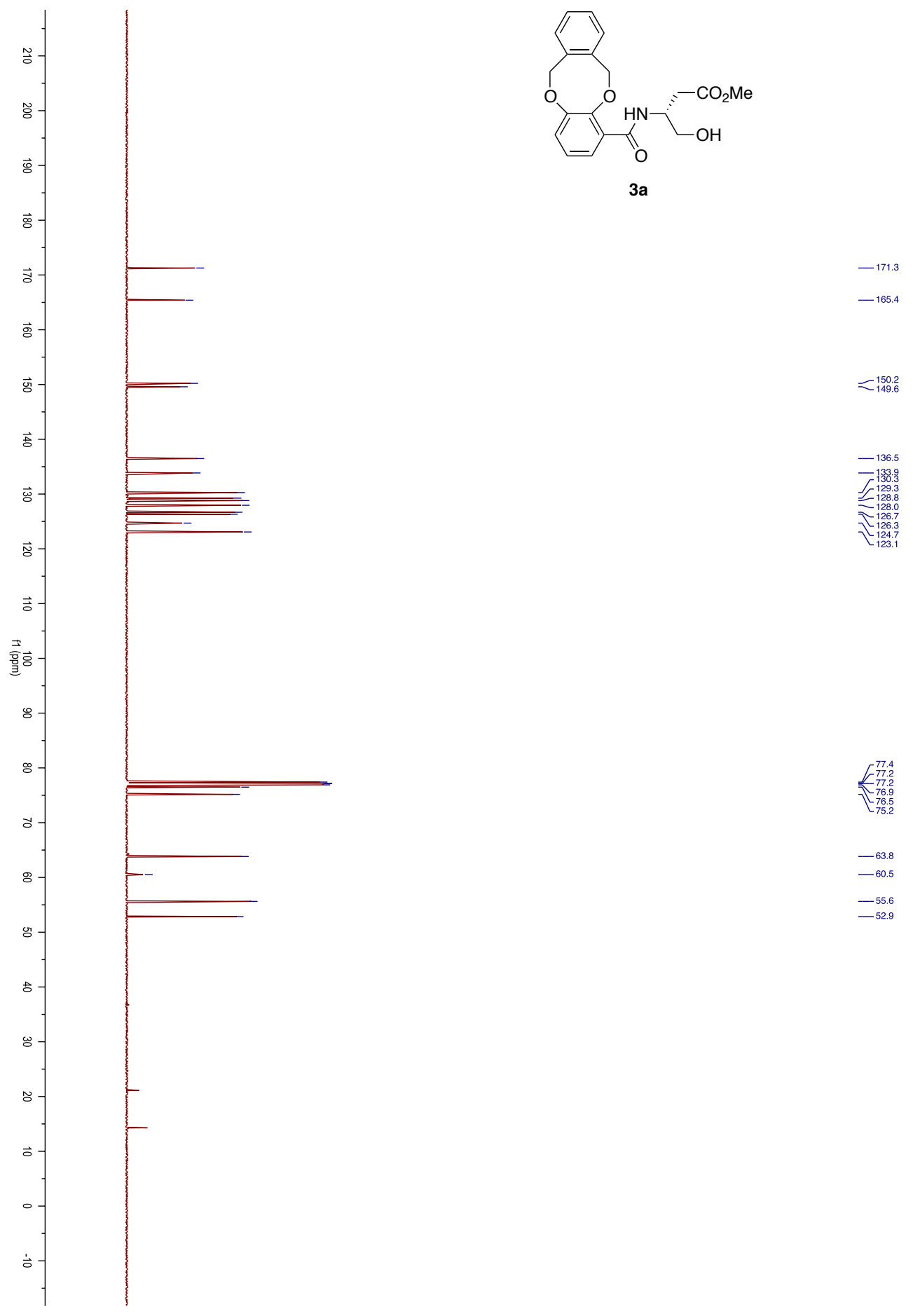


HR-MS of Compound 3a

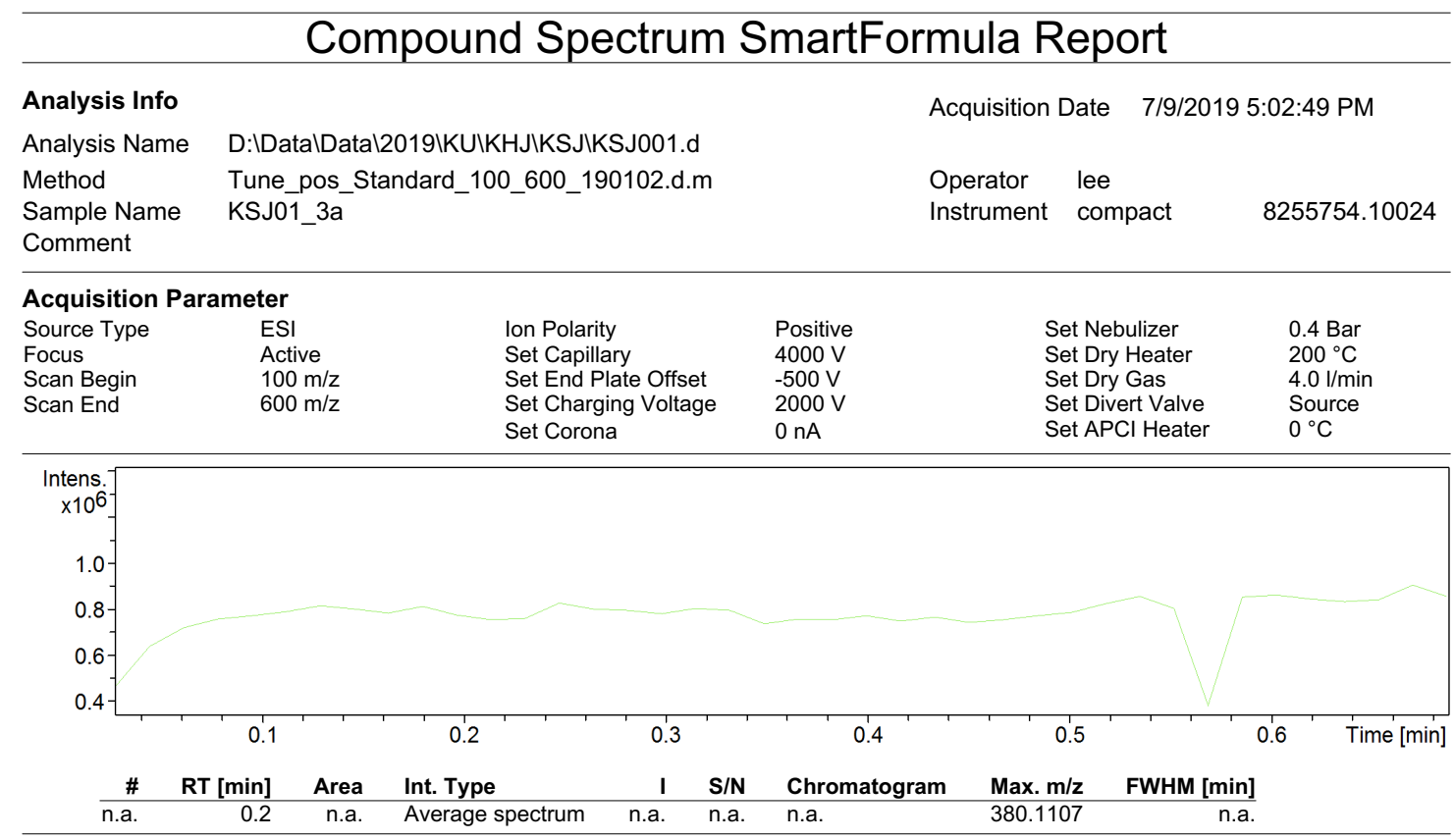

+MS, 0.1-0.4min \#3-23

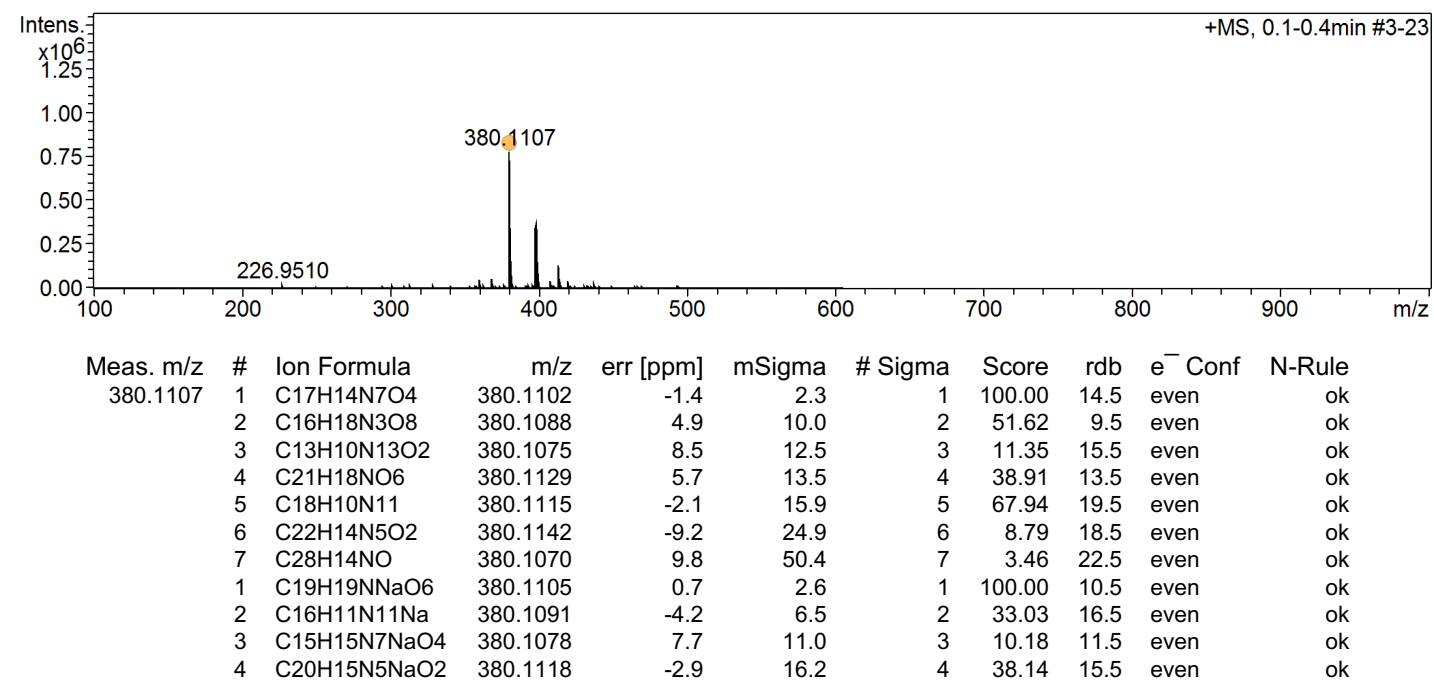




\section{${ }^{1} \mathrm{H}-\mathrm{NMR}$ of Compound 4a-i (500 $\left.\mathrm{MHz}, \mathrm{CDCl}_{3}\right)$}

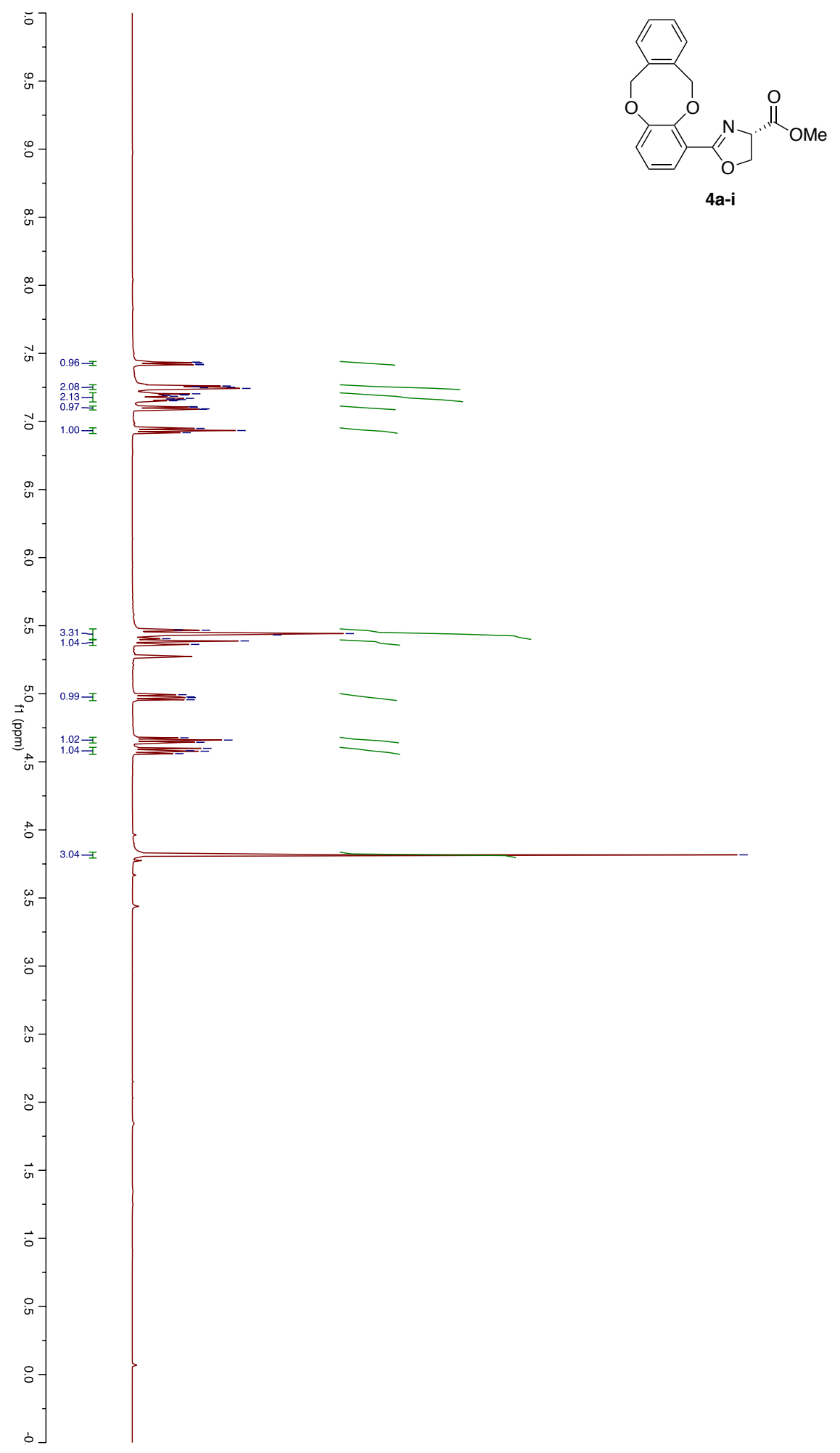


${ }^{13} \mathrm{C}-\mathrm{NMR}$ of Compound 4a-i (125 $\left.\mathrm{MHz}, \mathrm{CDCl}_{3}\right)$

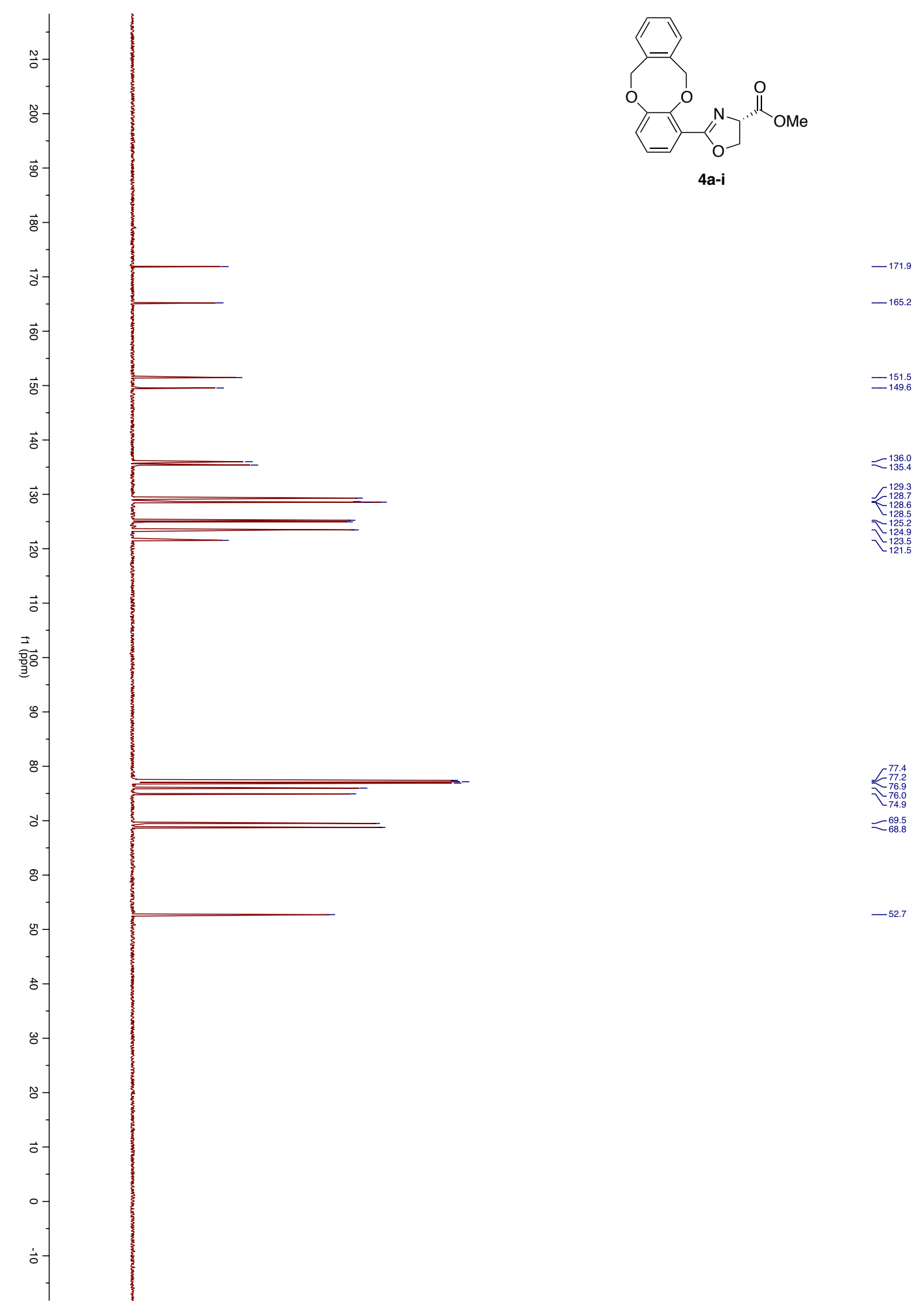


HR-MS of Compound 4a-i

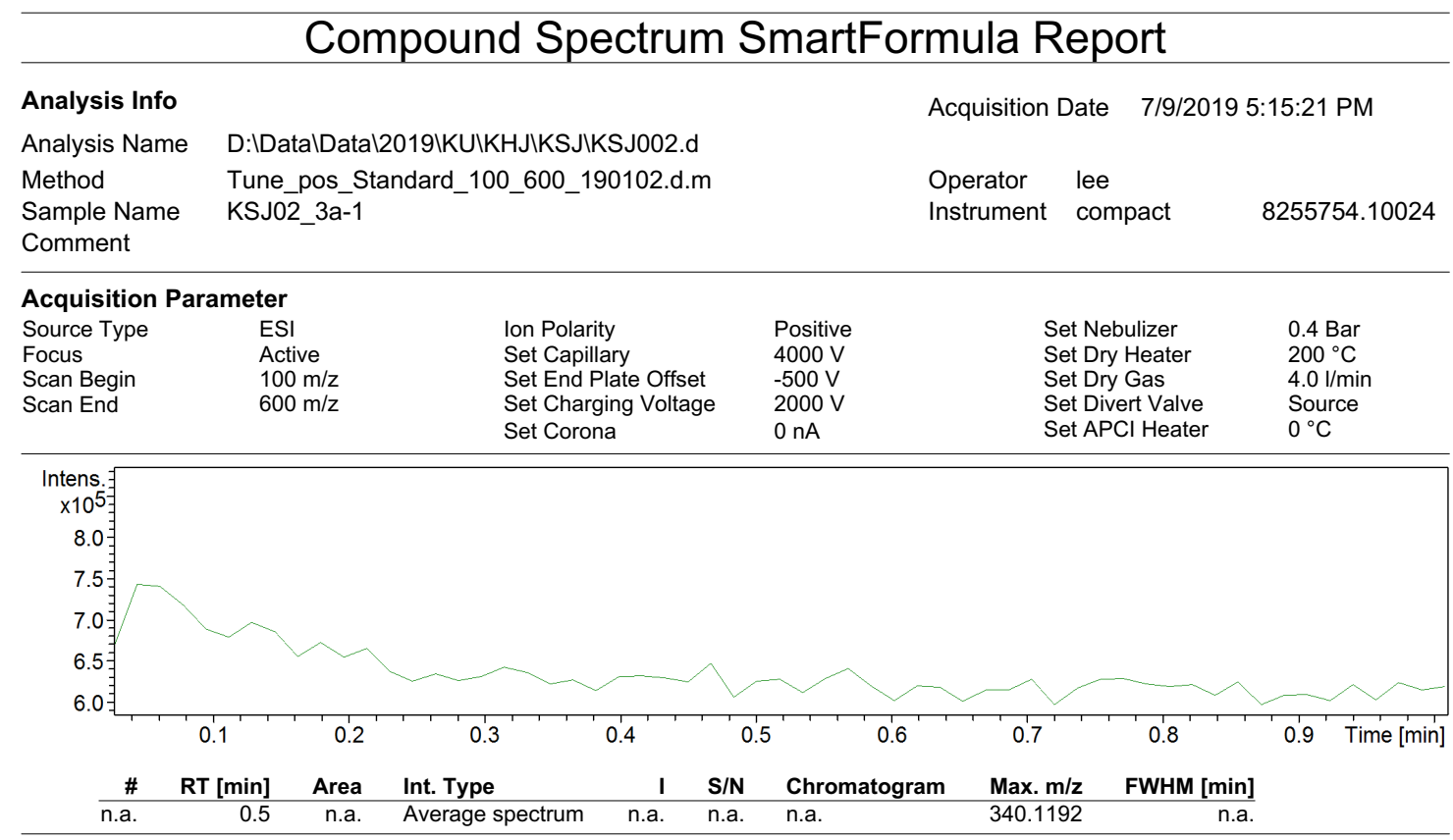

+MS, 0.3-0.7min \#17-42

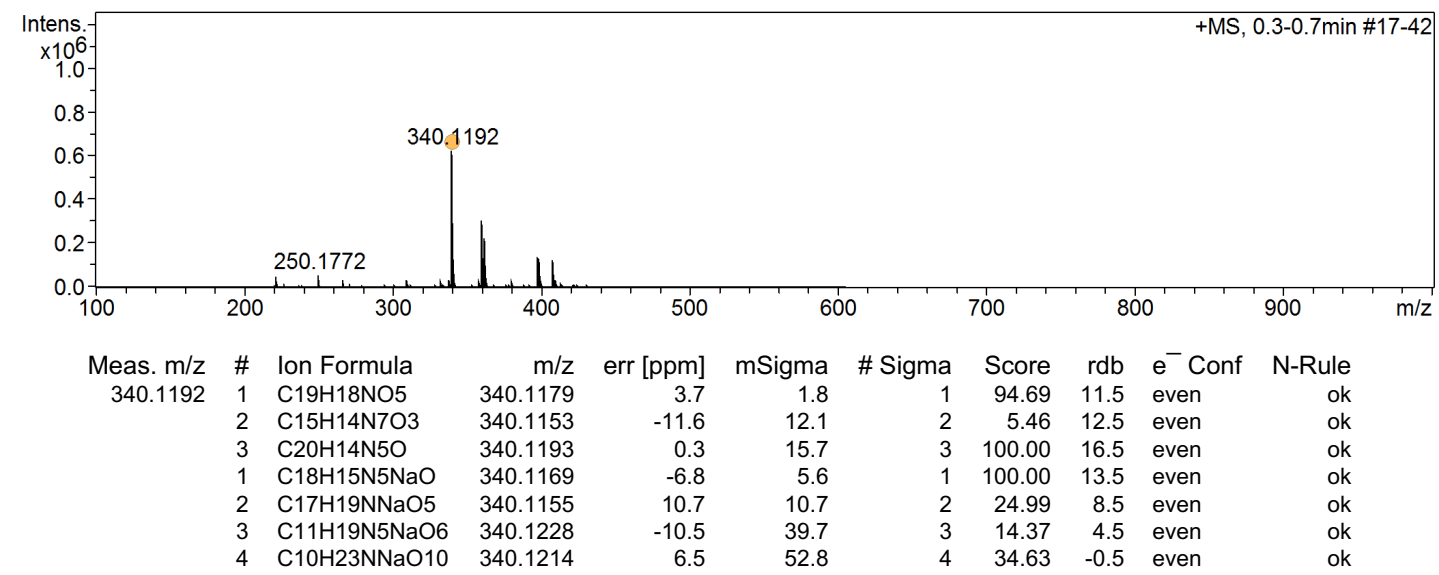

KSJ002.d

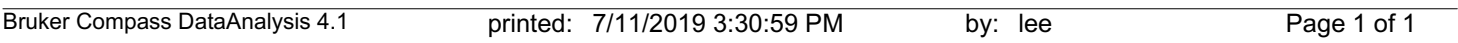




\section{${ }^{1} \mathrm{H}-\mathrm{NMR}$ of Compound 4a (500 MHz, $\left.\mathrm{CD}_{3} \mathrm{OD}\right)$}

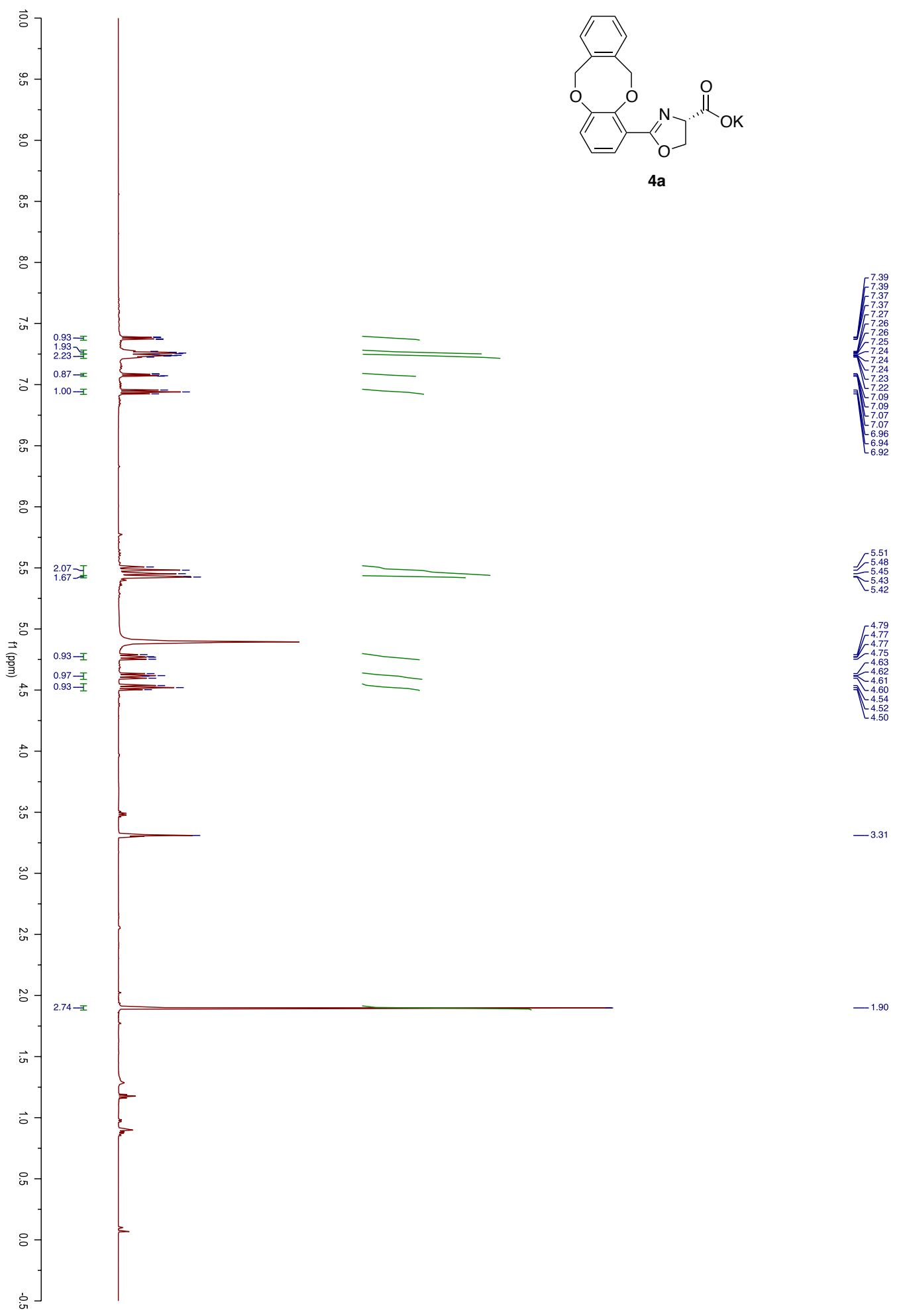




\section{${ }^{13} \mathrm{C}-\mathrm{NMR}$ of Compound 4a (125 MHz, $\left.\mathrm{CD}_{3} \mathrm{OD}\right)$}
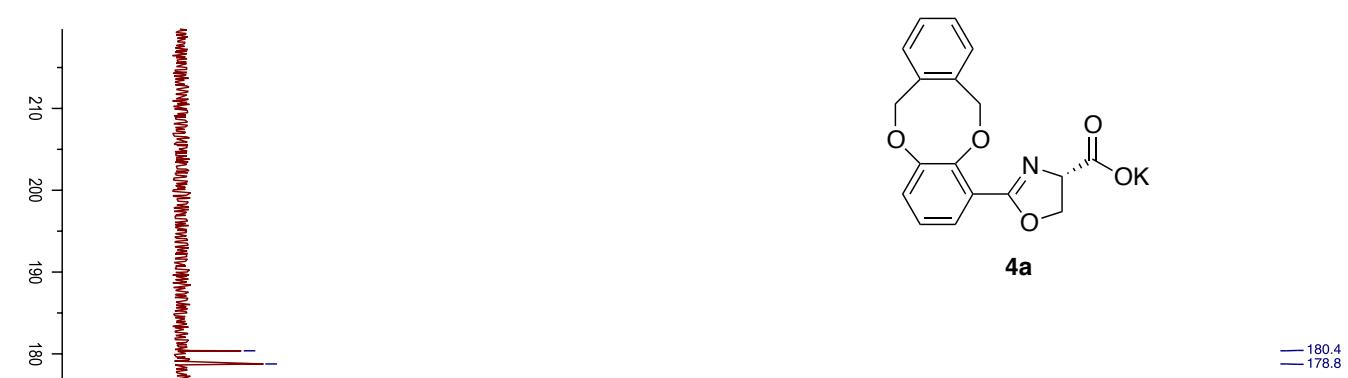

$-165.2$

二 $^{152.3}$

$\chi_{136.9}^{137.2}$

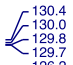

126.2
$\mathbf{Z}_{124.4}^{125}$
$\mathbf{n}_{124}$

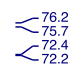

$\omega$

ะ

$\vec{o}$

o-

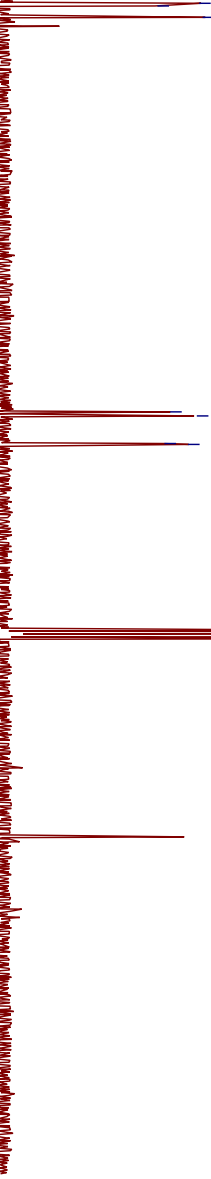


HR-MS of Compound 4a

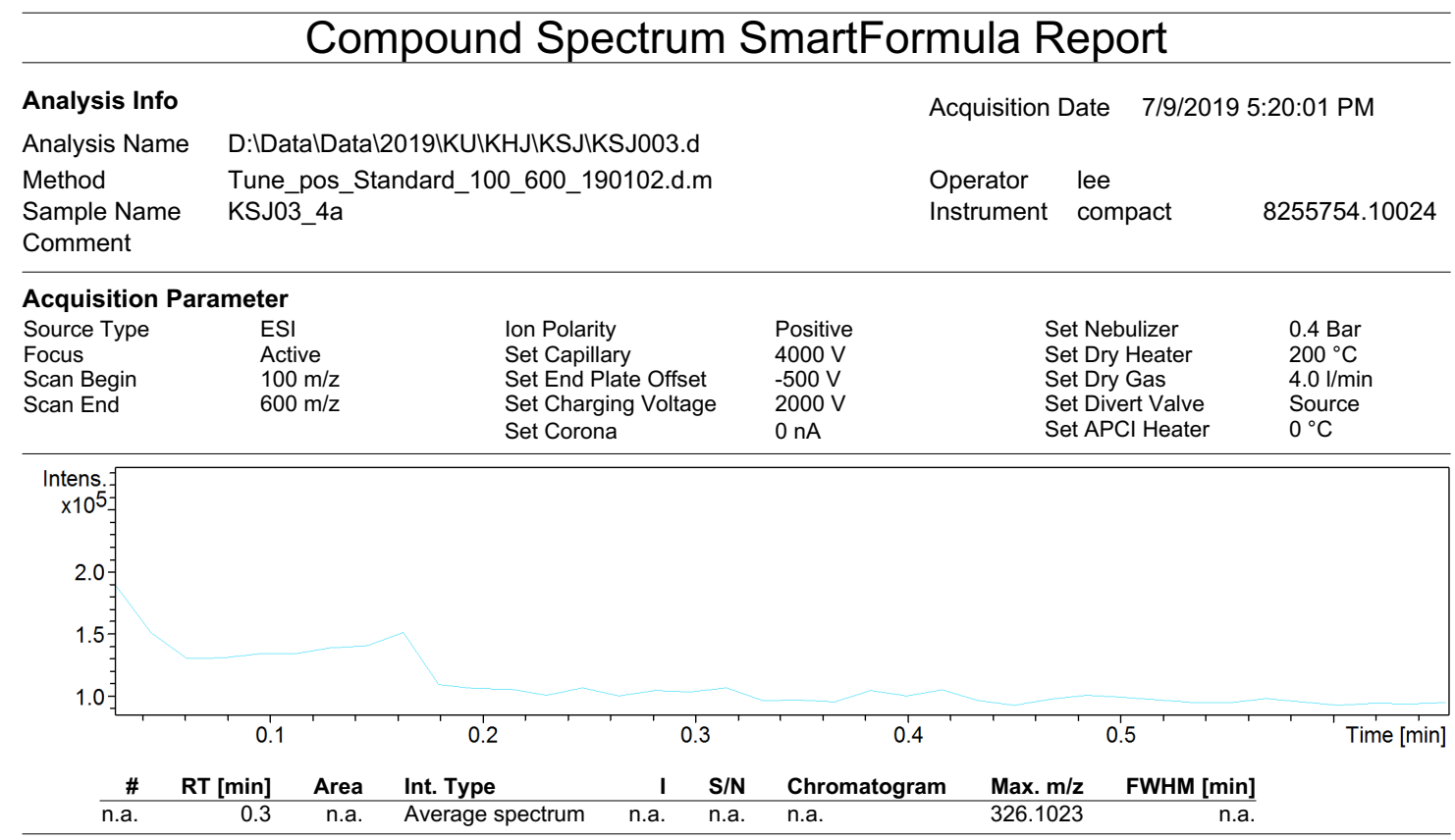

+MS, 0.1-0.5min \#4-27

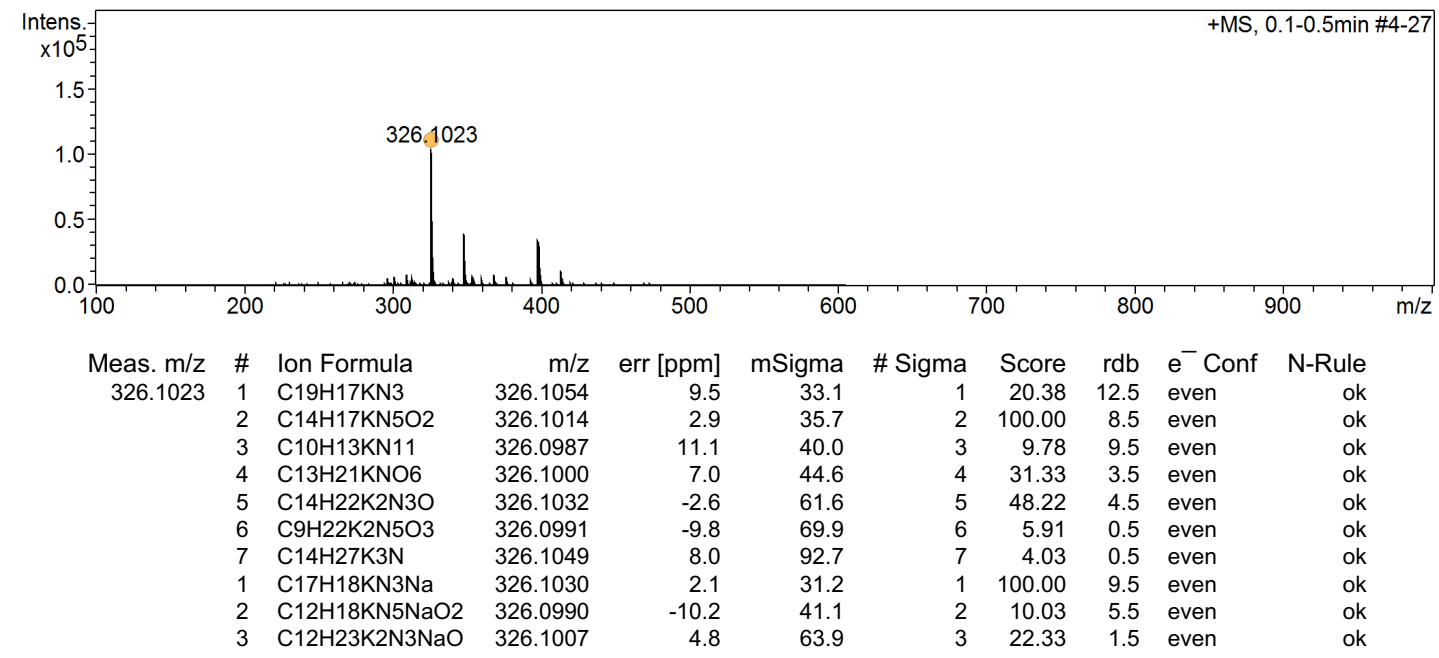

KSJ003.d

\begin{tabular}{llll}
\hline Bruker Compass DataAnalysis 4.1 & printed: & $7 / 11 / 2019$ 3:32:52 PM & by: lee
\end{tabular} 
${ }^{1} \mathrm{H}-\mathrm{NMR}$ of Compound 7a-i (500 $\left.\mathrm{MHz}, \mathrm{CDCl}_{3}\right)$
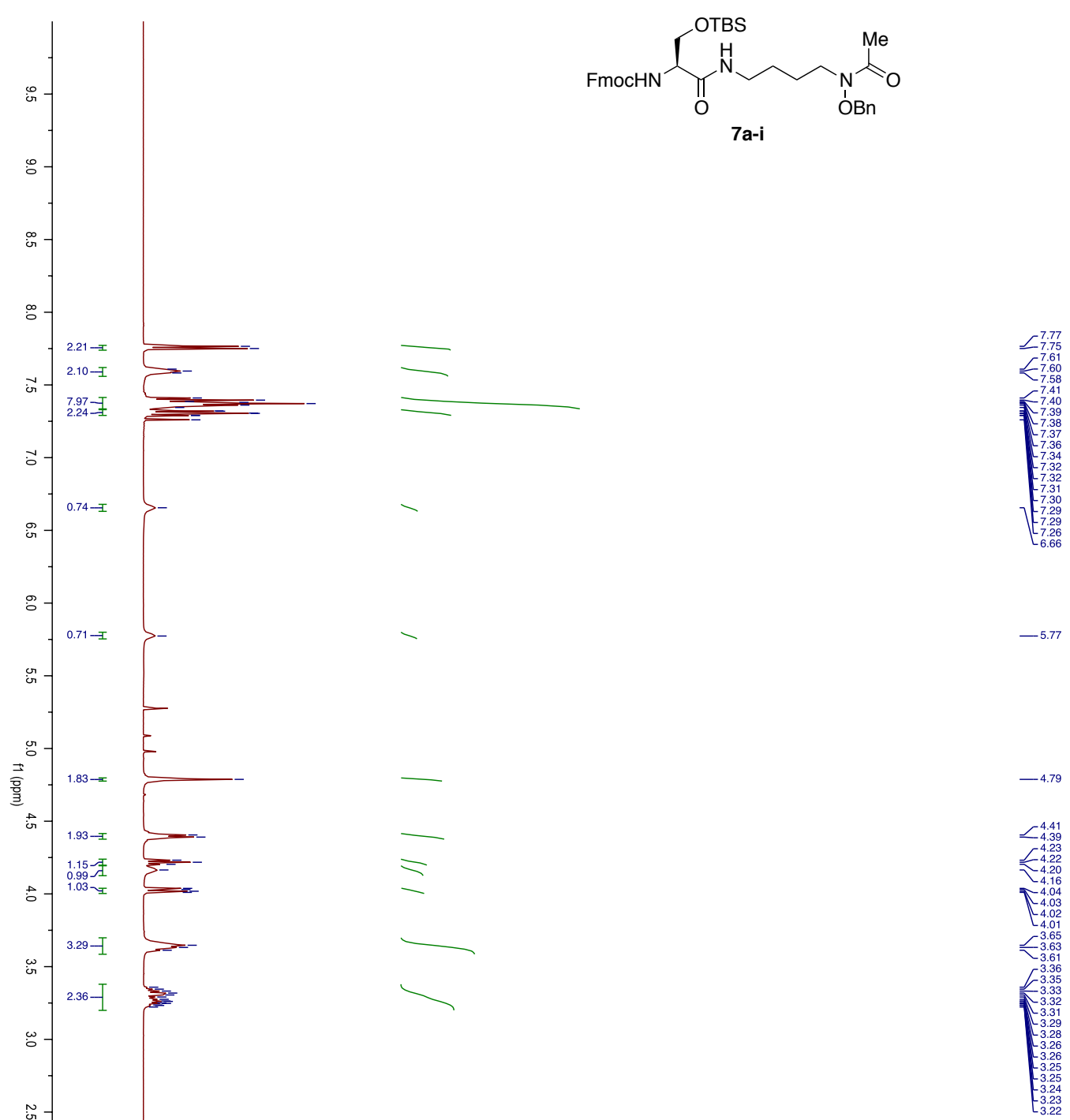

$-2.08$

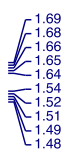

$-0.89$

$<_{0.07}^{0.08}$ 
${ }^{13} \mathrm{C}-\mathrm{NMR}$ of Compound 7a-i (125 MHz, CDCl3)
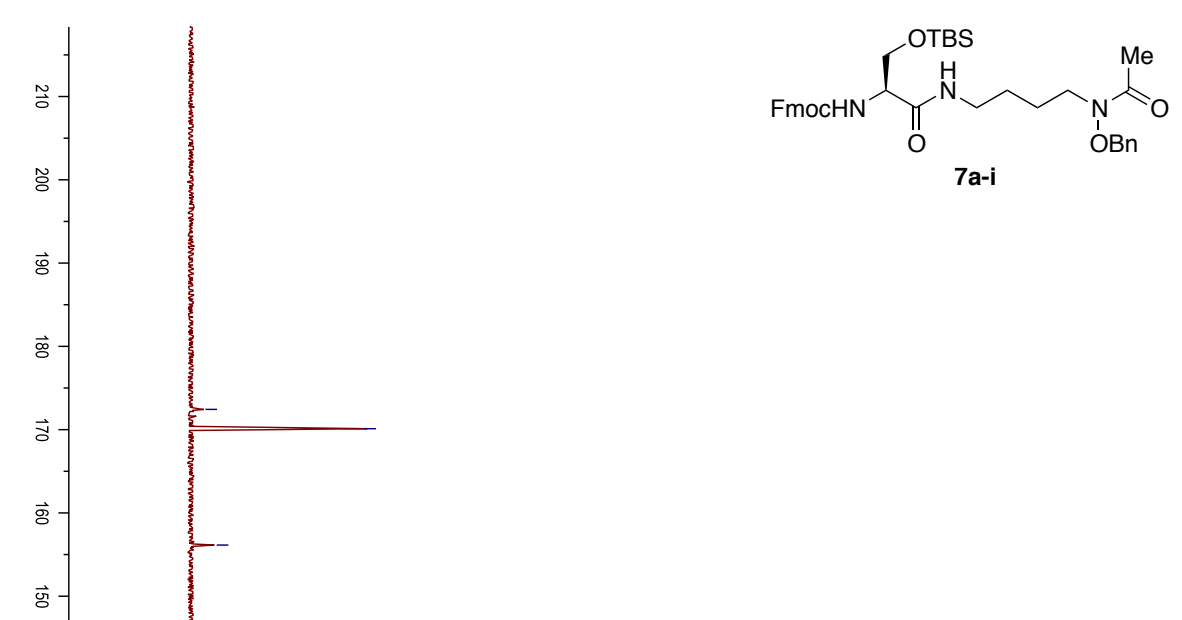

$-156.1$

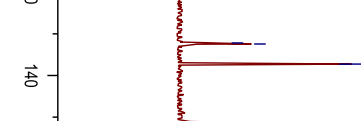

$<_{143.8}^{143.9}$

143.8
$\mathbf{X}_{141.4}^{14.4}$

$-134.4$

F

$\overrightarrow{\mathrm{s}}$

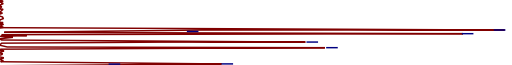

$\overrightarrow{\tilde{\sigma}}$

$$
\overrightarrow{\mathrm{o}}
$$$$
\text { 홀홍 }
$$$$
8
$$

$\infty$

o

강-

s

$\omega$

$\approx$

$\vec{\circ}$

$\circ$

के

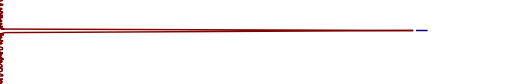

-67.2

- 63.3

$-55.8$

${ }^{4} 4.2 .8$

$-39.3$

$\mathbf{Z}_{22.9}^{26.6}$

${ }^{20.5}$

$<_{-5.5}^{5.4}$ 
HR-MS of Compound 7a-i

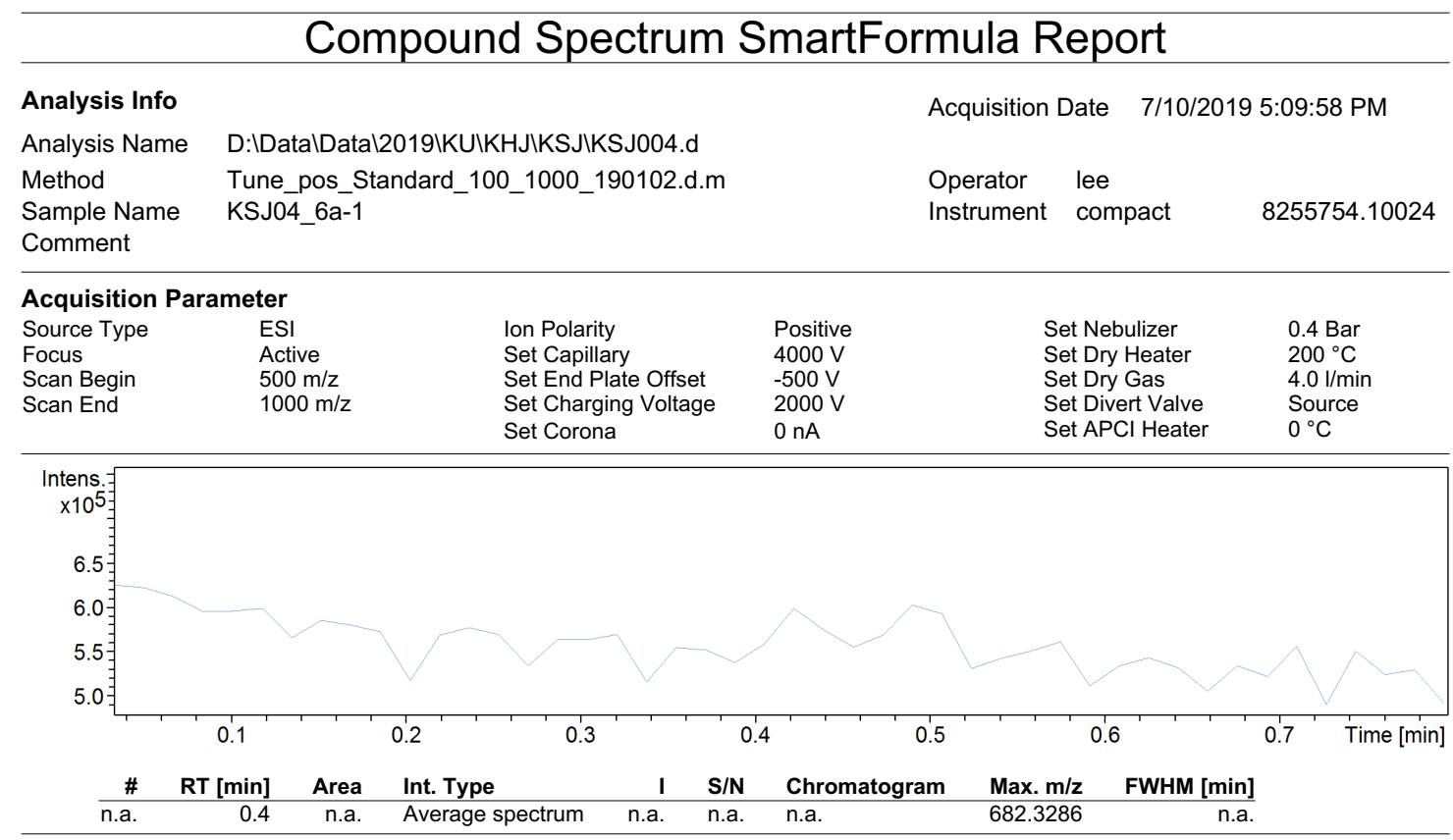

+MS, 0.1-0.7min \#6-39

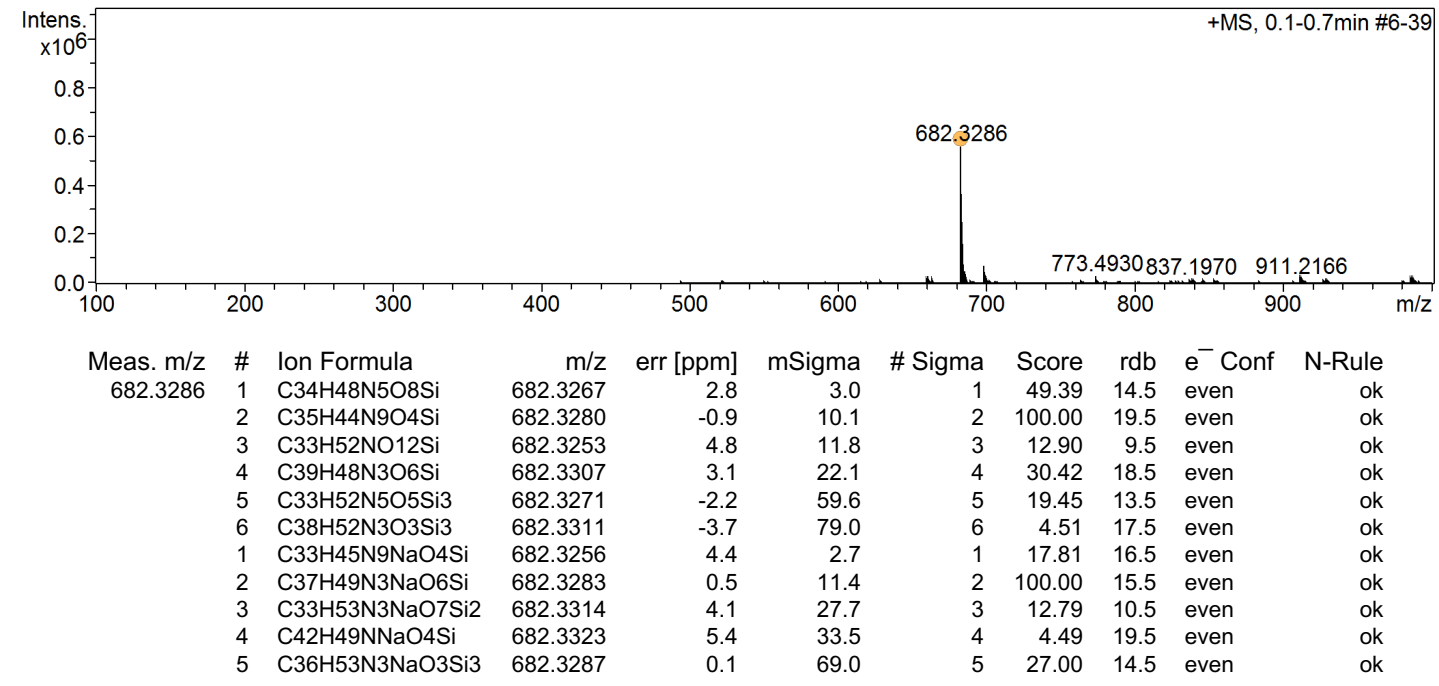

KSJ004.d

Bruker Compass DataAnalysis 4.1 $\quad$ printed: 7/11/2019 3:38:06 PM $\quad$ by: lee 1 Page 1 of 
${ }^{1} \mathrm{H}-\mathrm{NMR}$ of Compound 7a (500 $\left.\mathrm{MHz}, \mathrm{CDCl}_{3}\right)$

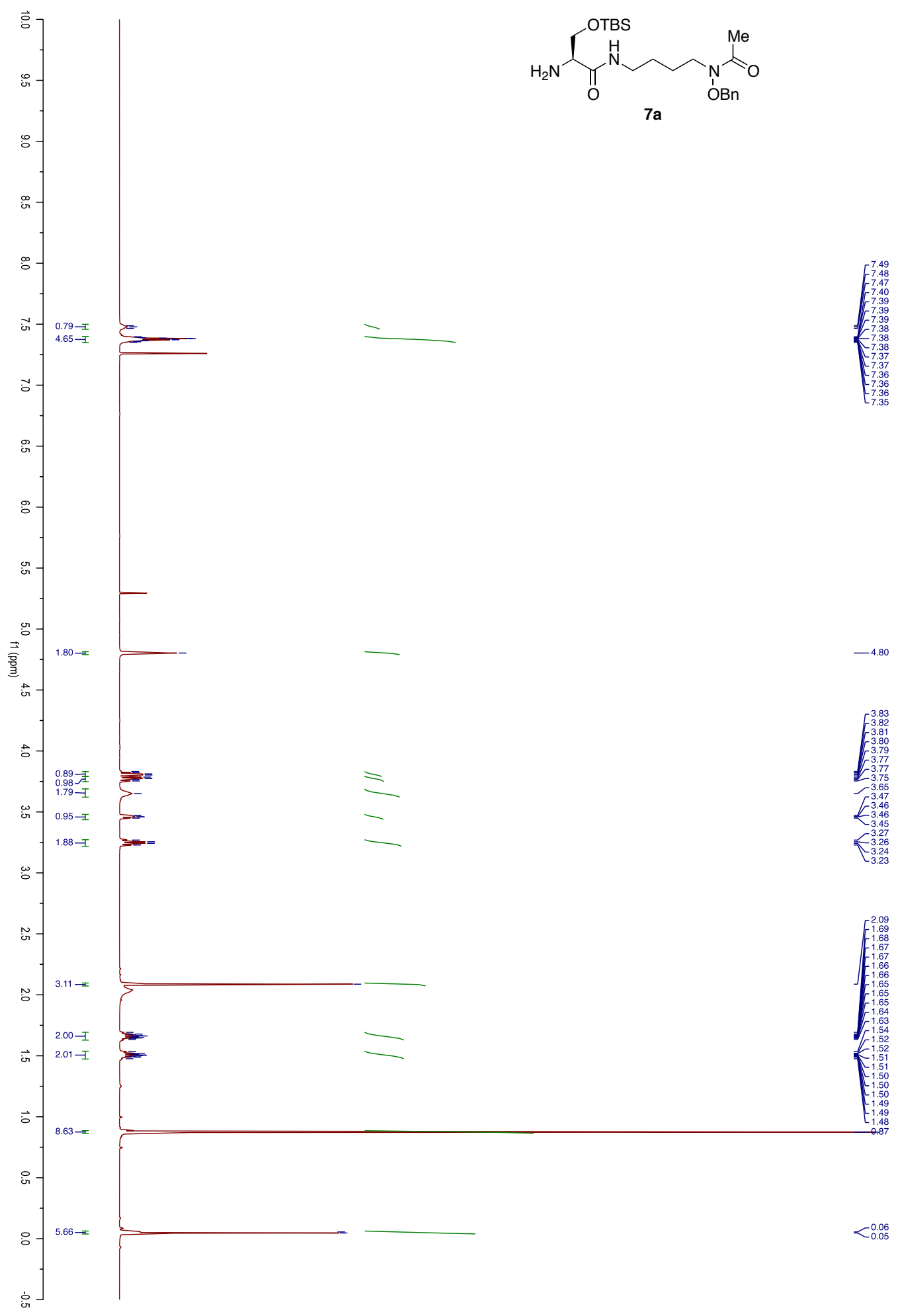


${ }^{13} \mathrm{C}-\mathrm{NMR}$ of Compound $7 \mathrm{a}\left(125 \mathrm{MHz}, \mathrm{CDCl}_{3}\right)$

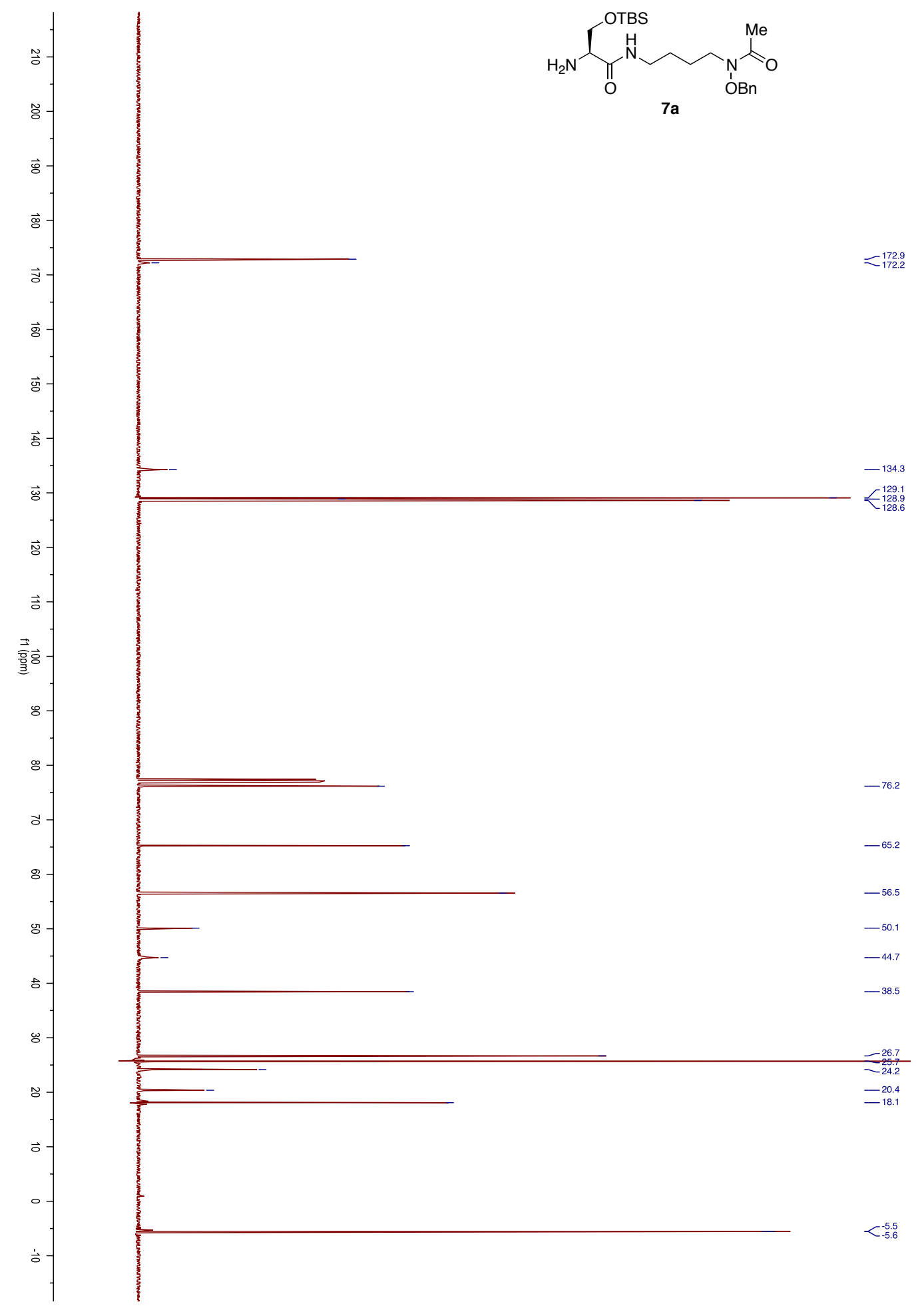


HR-MS of Compound 7a

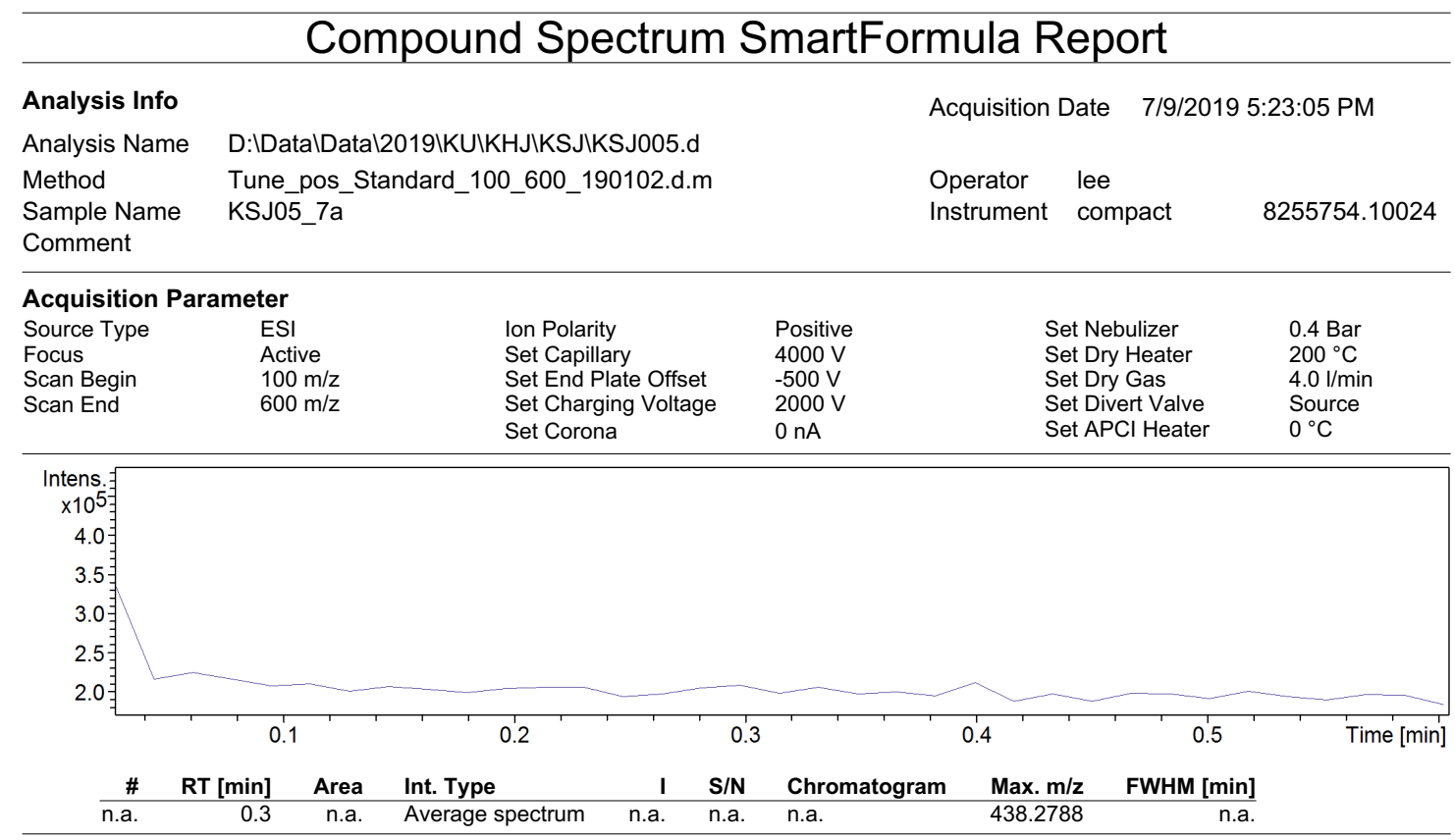

+MS, 0.1-0.4min \#5-26

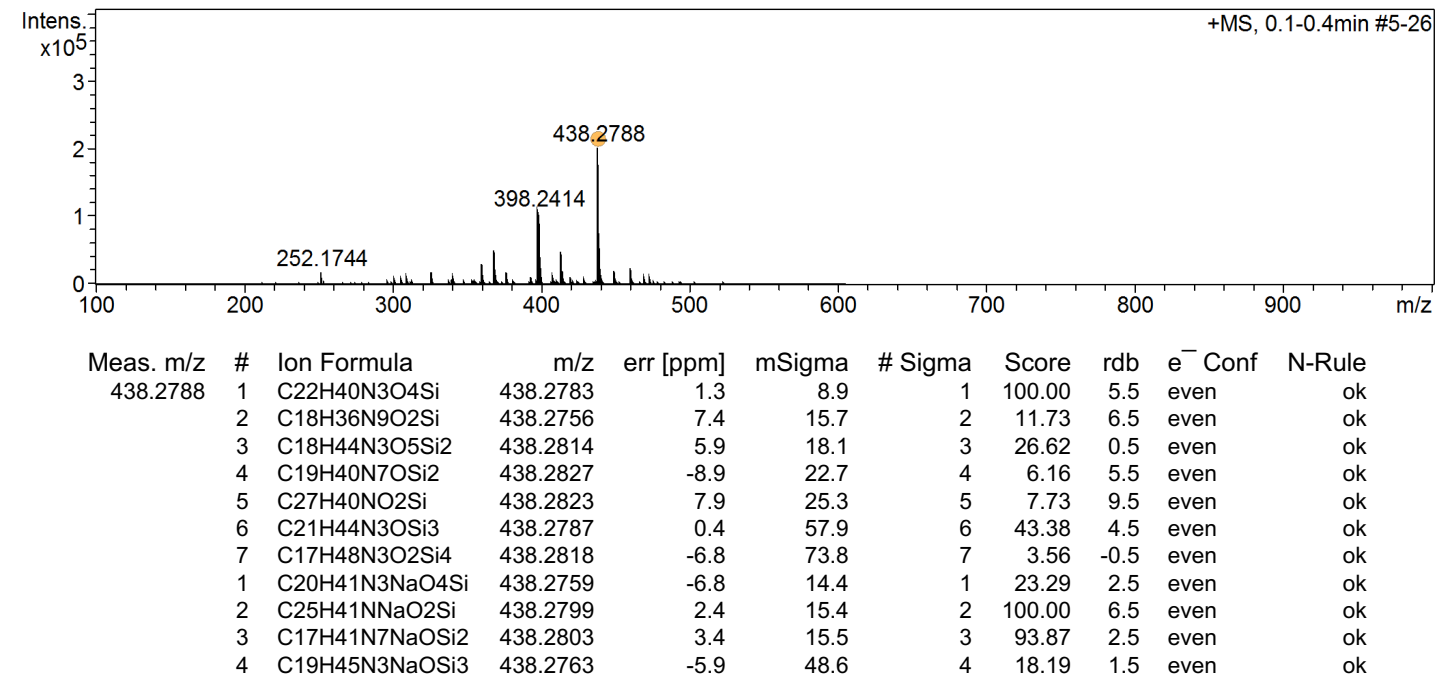

KSJ005.d

Bruker Compass DataAnalysis 4.1 $\quad$ printed: 7/11/2019 3:39:49 PM $\quad$ by: lee 1 Page 1 of 
${ }^{1} \mathrm{H}-\mathrm{NMR}$ of Compound 8a (500 MHz, CD $\mathrm{OD}$ )

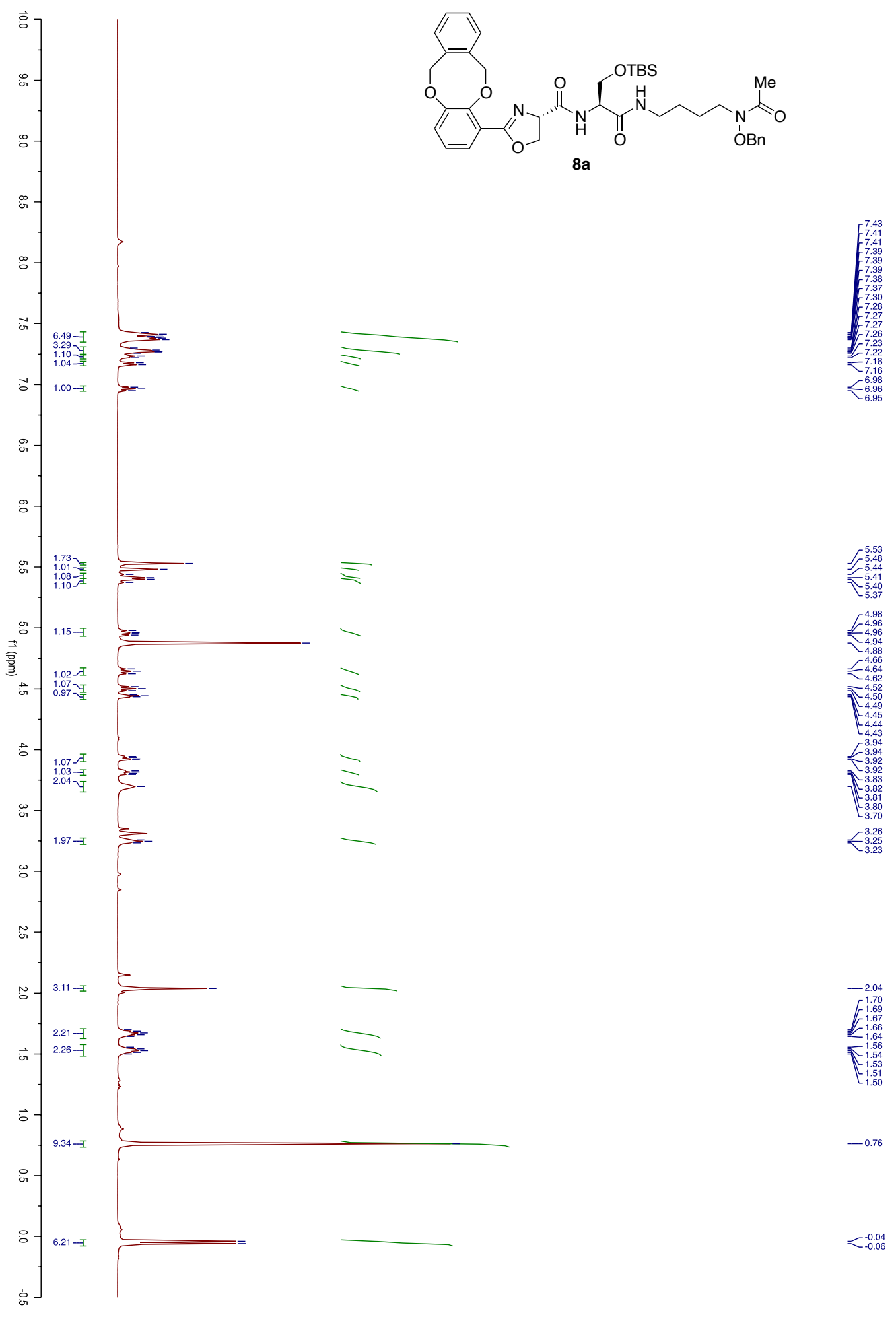




\section{${ }^{13} \mathrm{C}-\mathrm{NMR}$ of Compound 8a (125 MHz, $\left.\mathrm{CD}_{3} \mathrm{OD}\right)$}
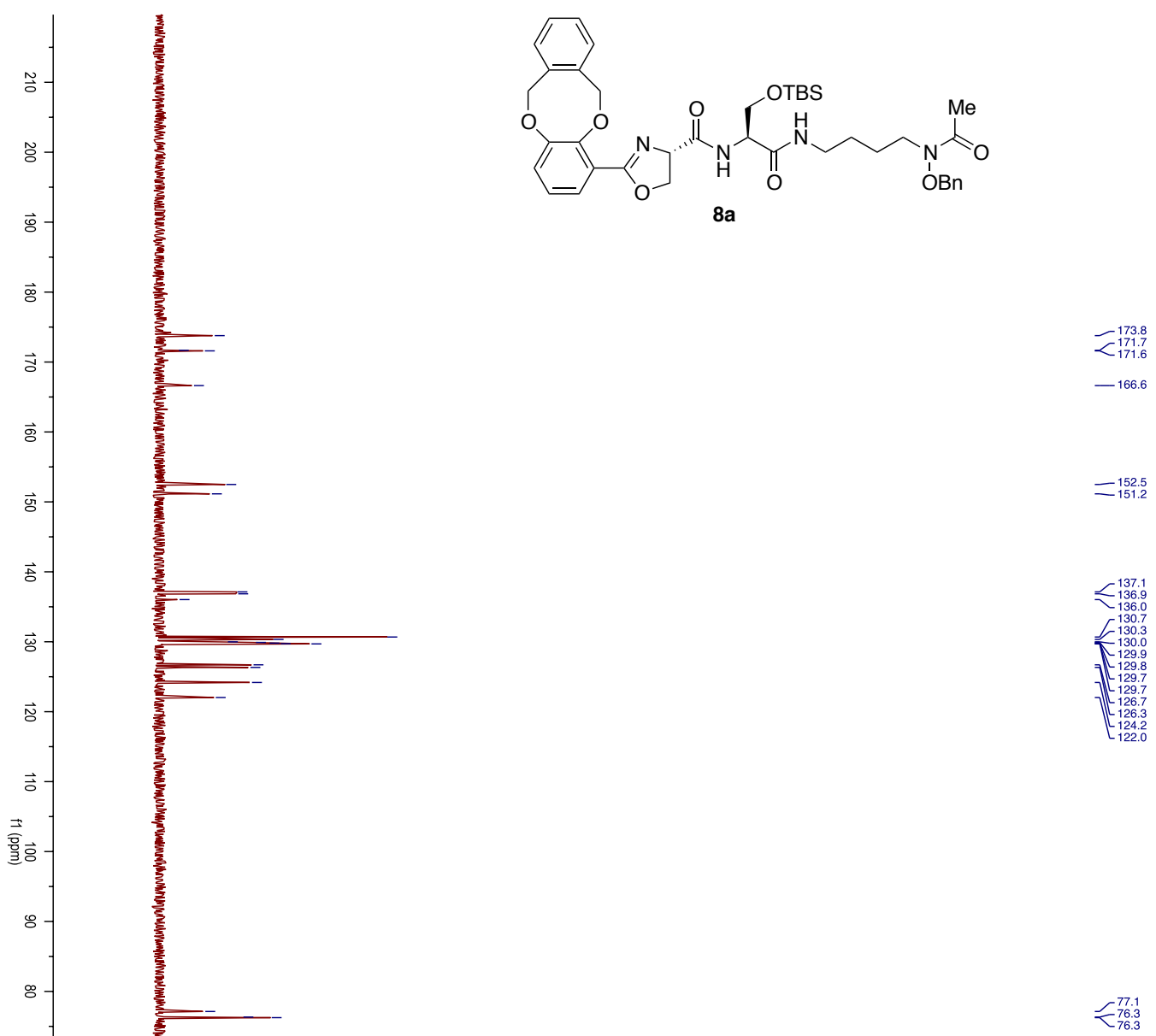

$\mathbf{C}_{70.3}^{71.1}$

$-64.4$

$-56.3$ 
HR-MS of Compound 8a

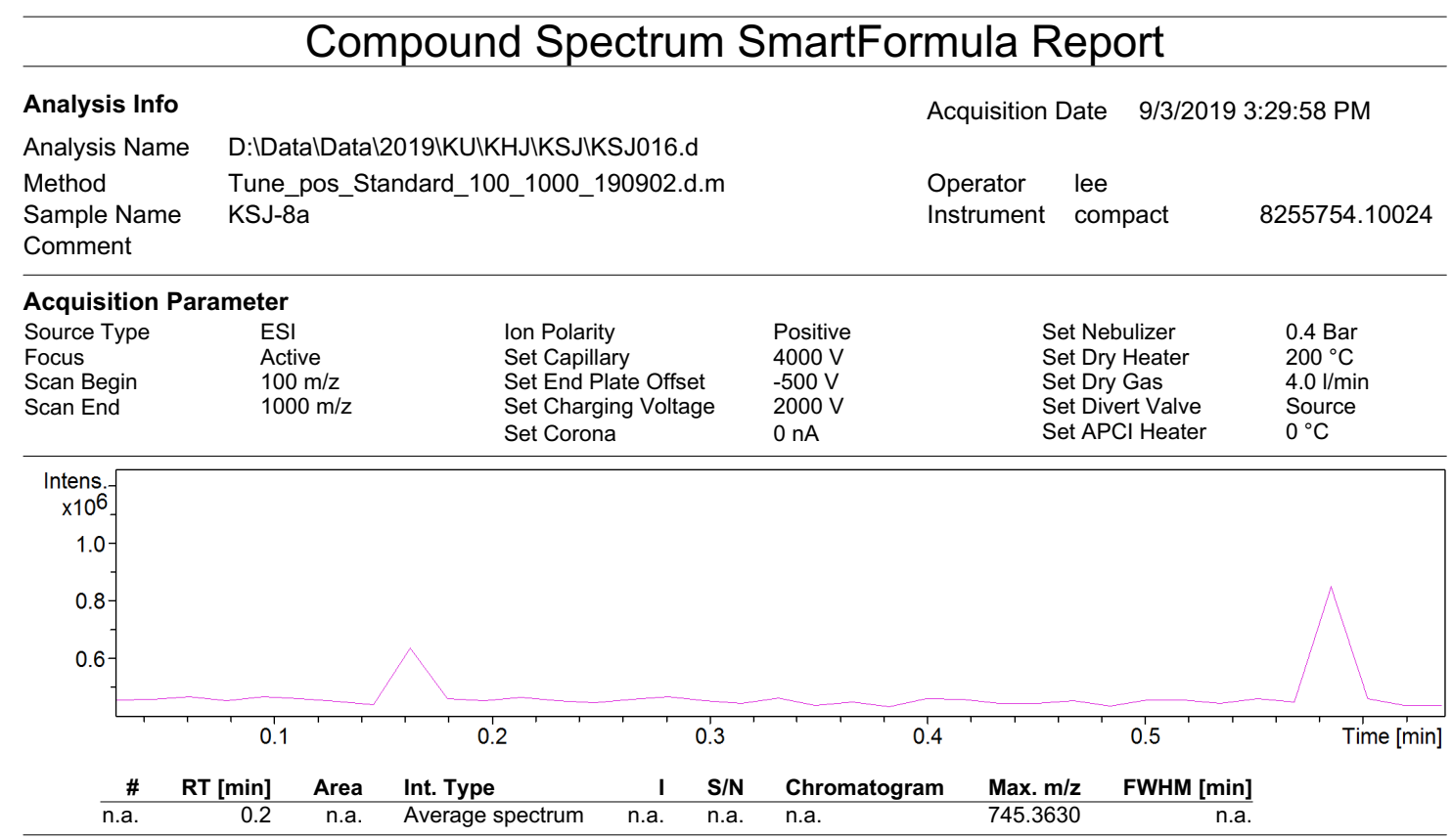

+MS, 0.1-0.4min \#3-21

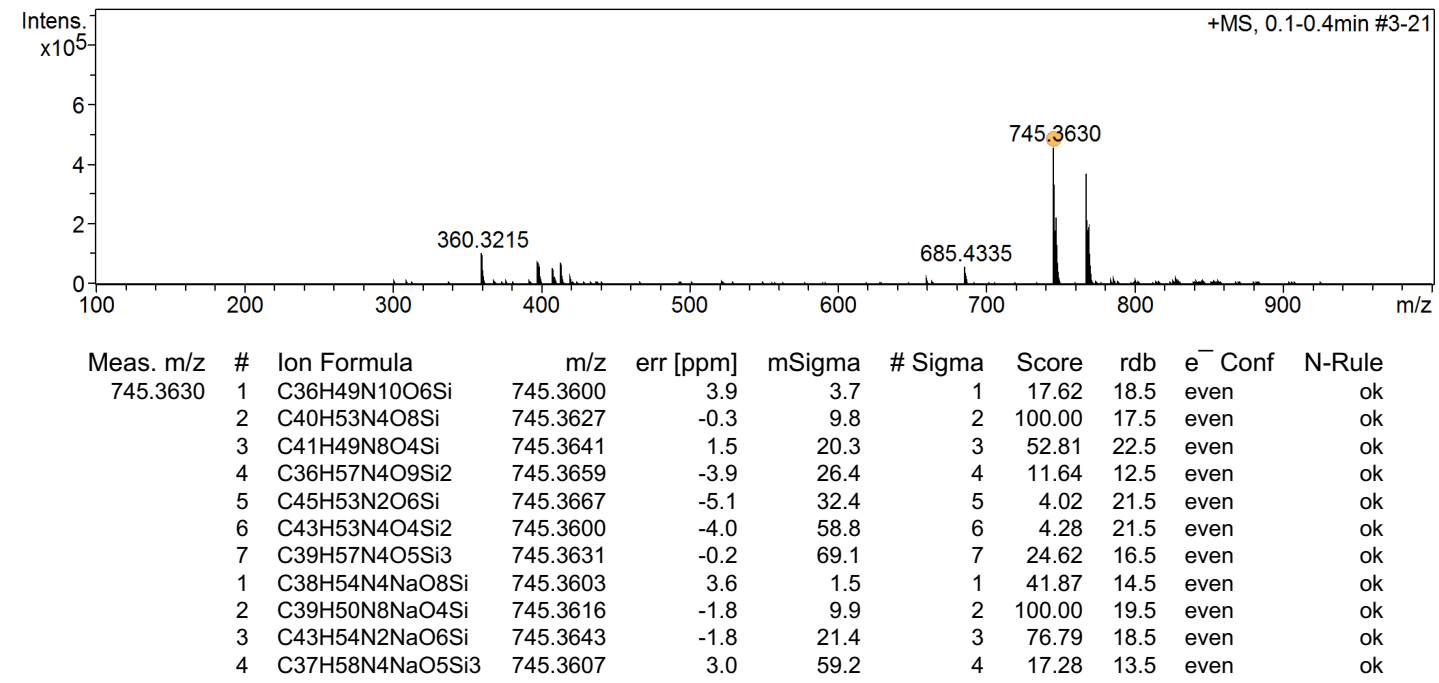

KSJ016.d

Bruker Compass DataAnalysis 4.1 $\quad$ printed: 9/3/2019 3:37:49 PM $\quad$ by: lee 1 Page 1 of 
${ }^{1} \mathrm{H}-\mathrm{NMR}$ of Compound $8 \mathrm{~b}\left(500 \mathrm{MHz}, \mathrm{CD}_{3} \mathrm{OD}\right)$

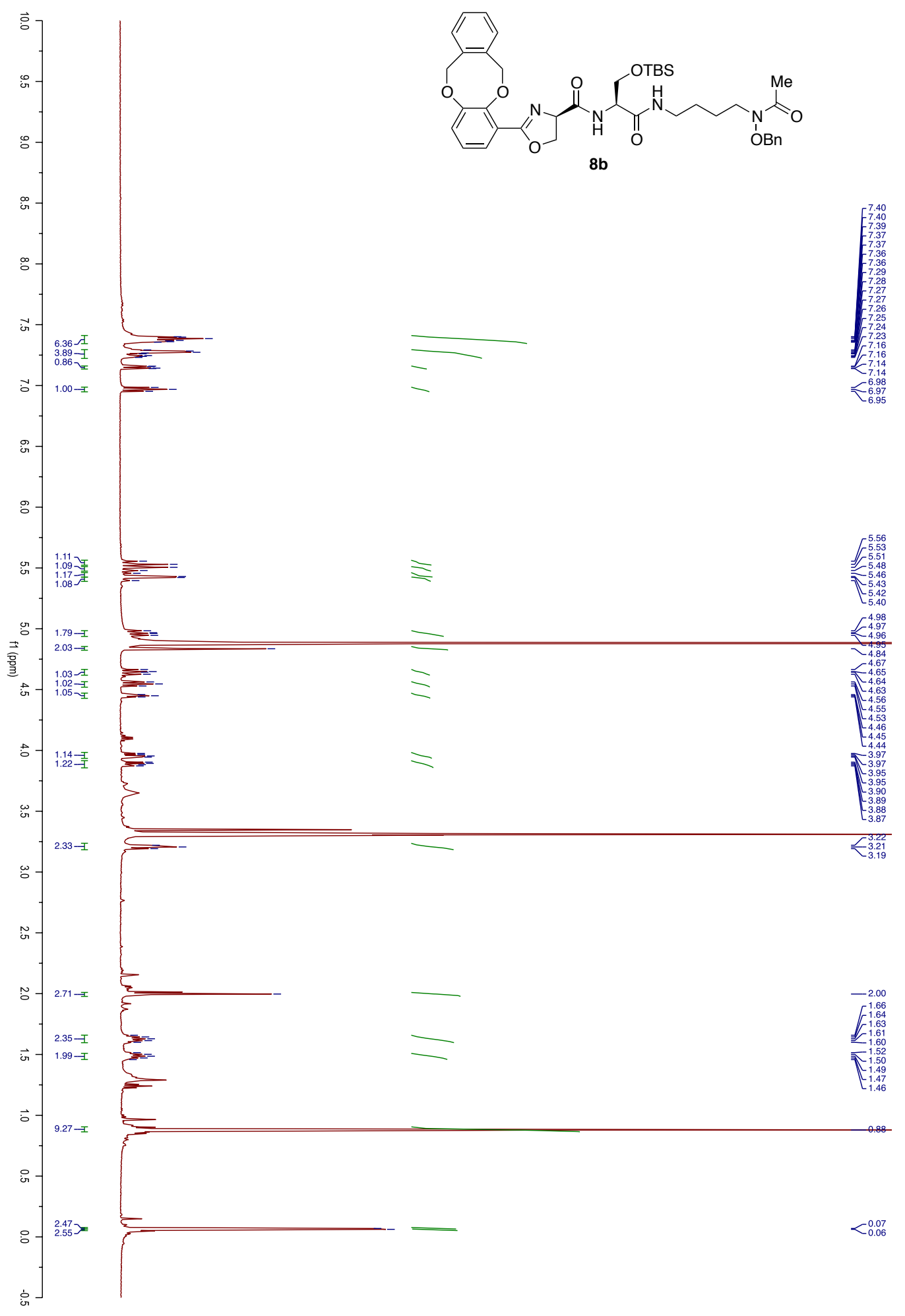




\section{${ }^{13} \mathrm{C}-\mathrm{NMR}$ of Compound $8 \mathrm{~b}\left(125 \mathrm{MHz}, \mathrm{CD}_{3} \mathrm{OD}\right)$}

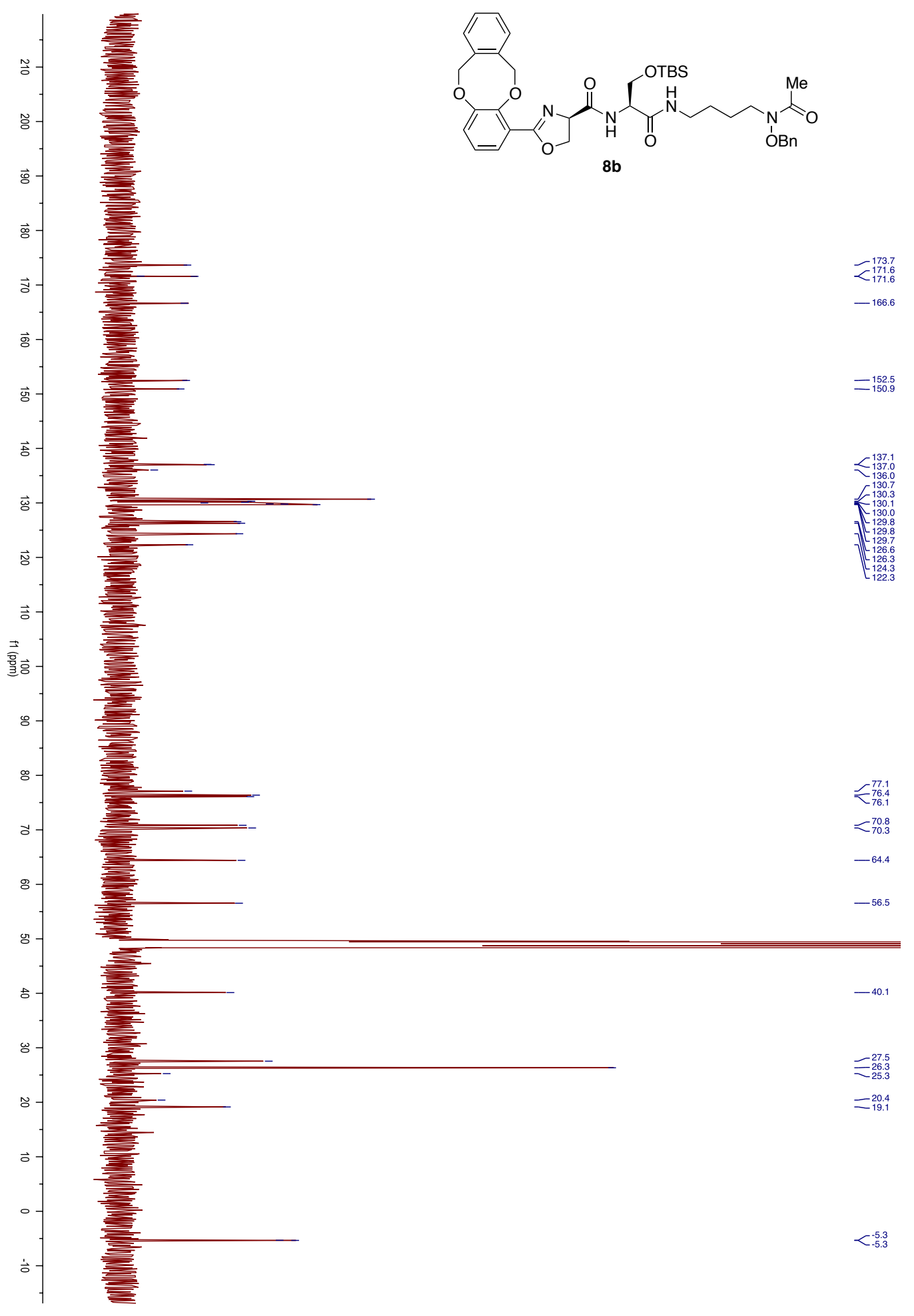


HR-MS of Compound $8 \mathrm{~b}$

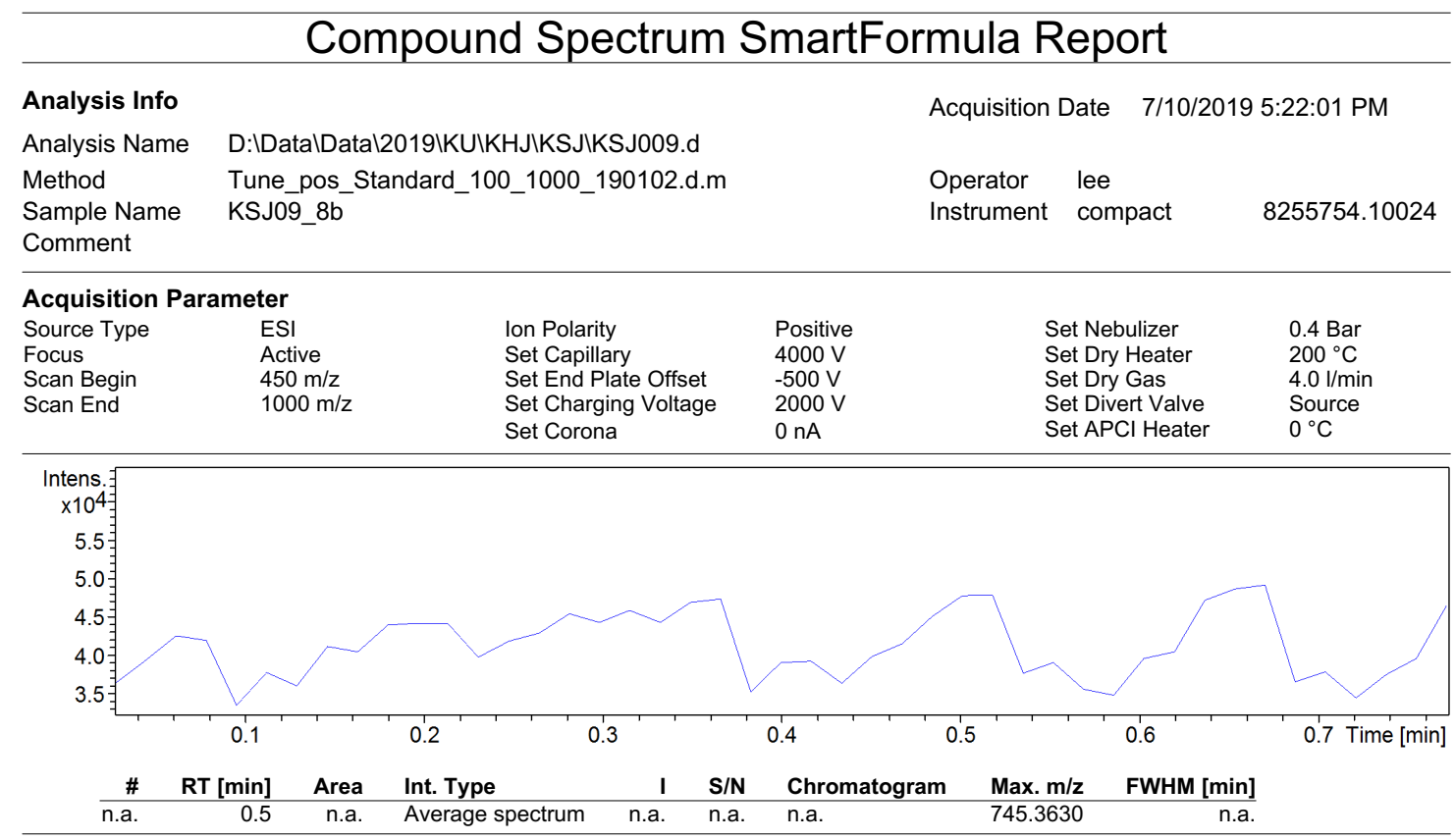

+MS, 0.2-0.7min \#9-43

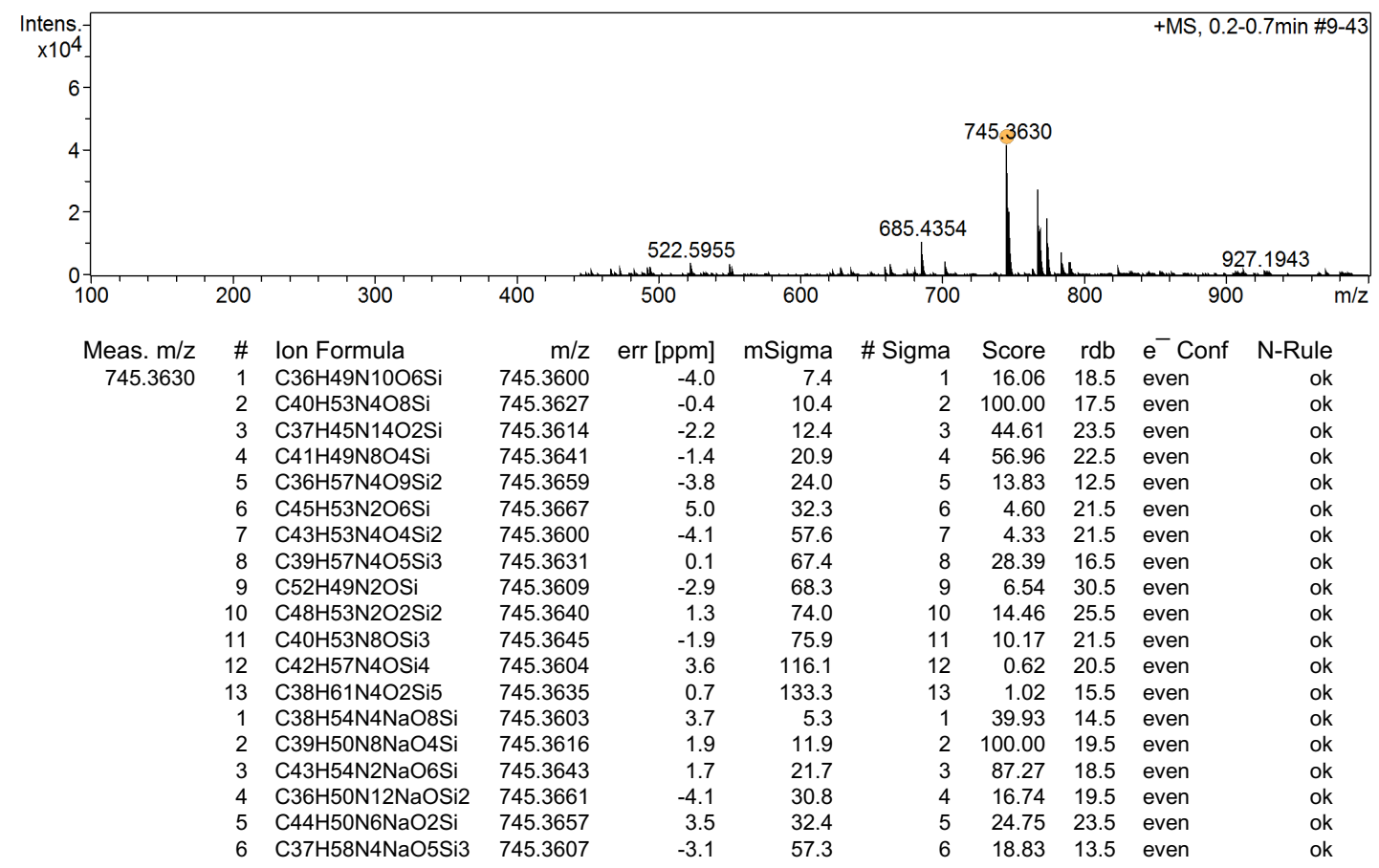

KSJ009.d

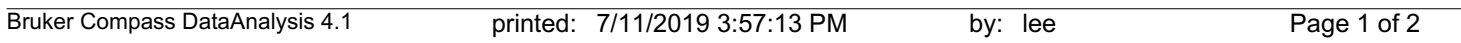


${ }^{1} \mathrm{H}-\mathrm{NMR}$ of Compound $9\left(500 \mathrm{MHz}, \mathrm{CDCl}_{3}\right)$

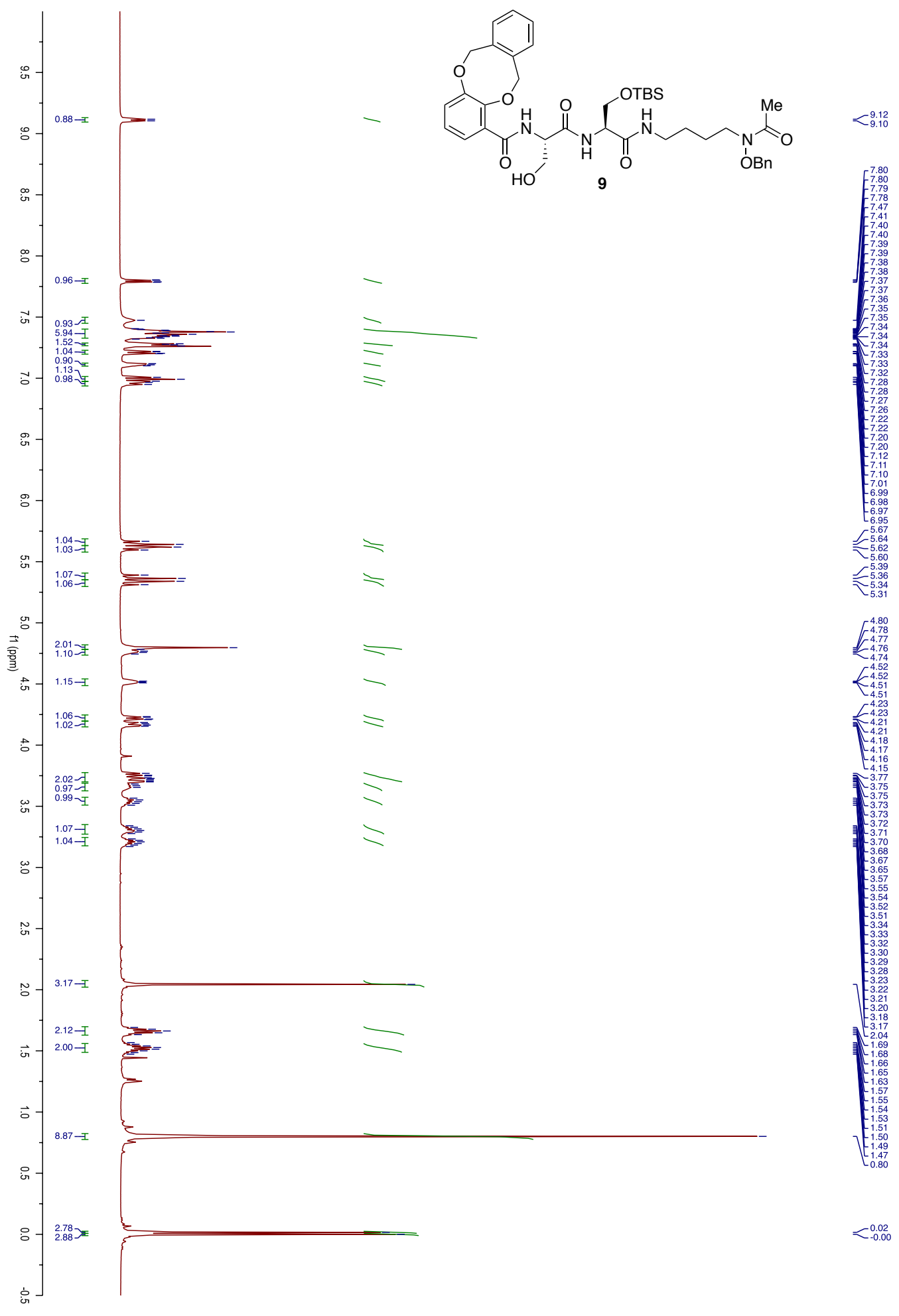


${ }^{13} \mathrm{C}-\mathrm{NMR}$ of Compound 9 (125 MHz, $\left.\mathrm{CDCl}_{3}\right)$

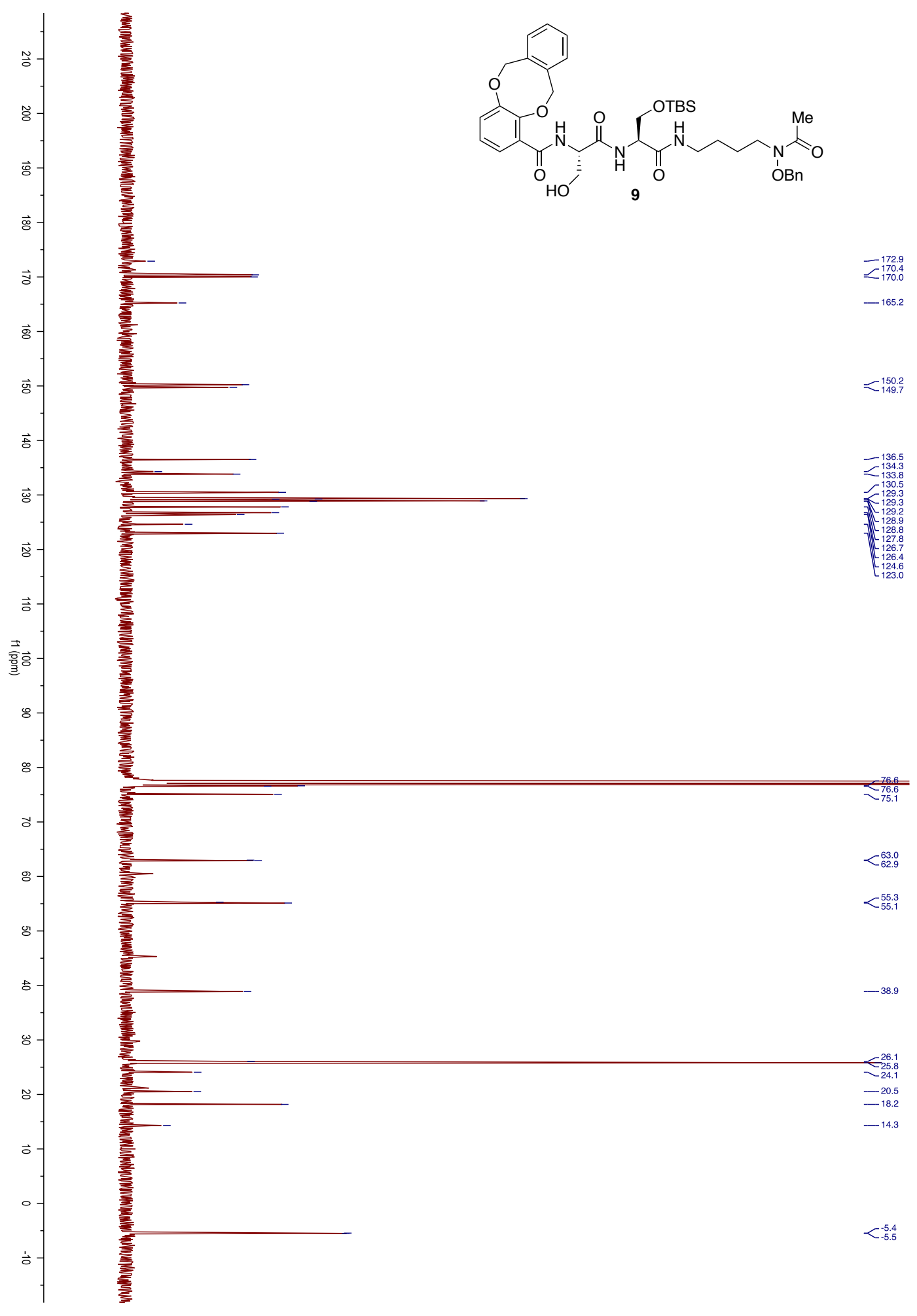


HR-MS of Compound 9

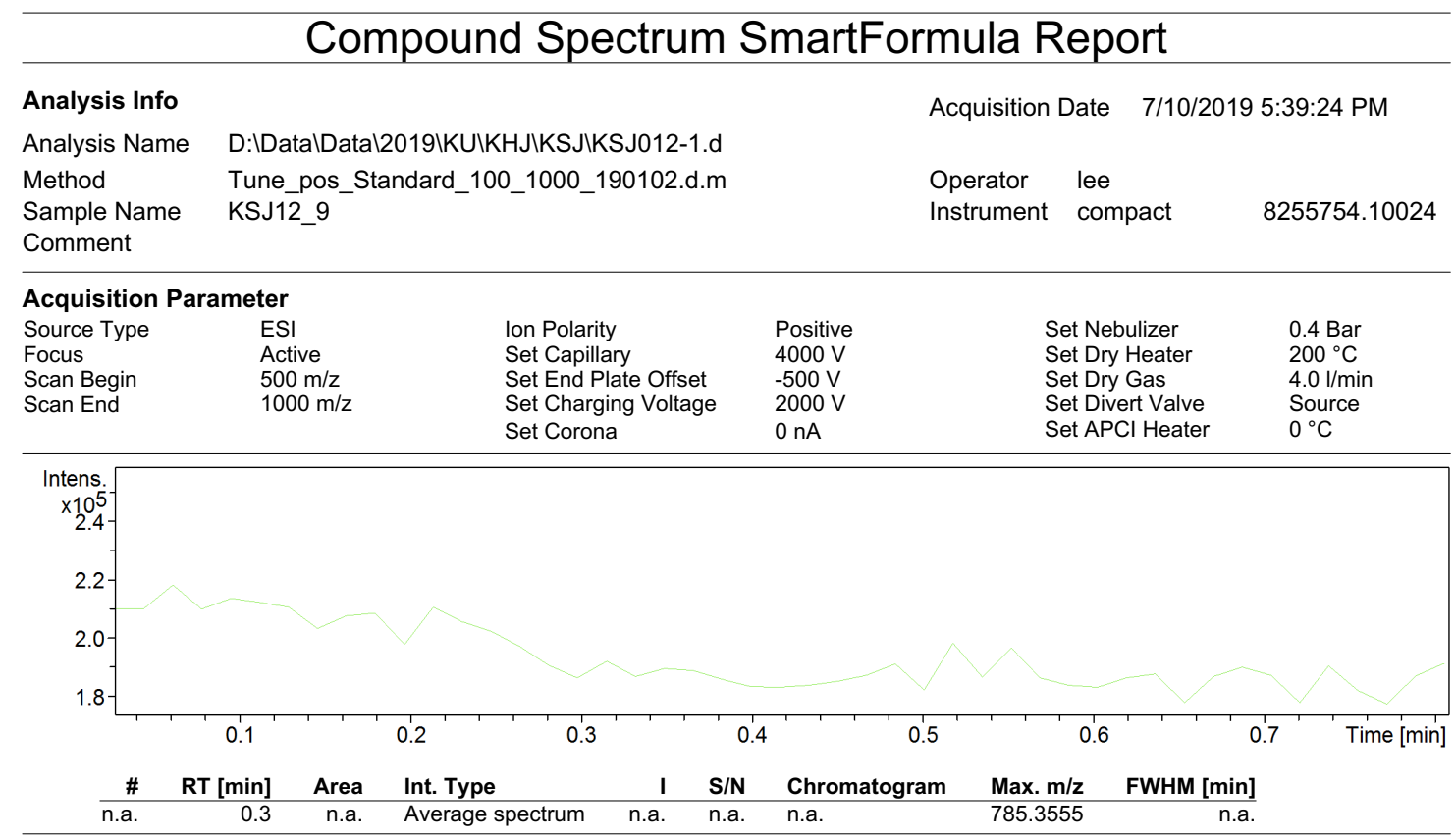

+MS, 0.1-0.6min \#5-32

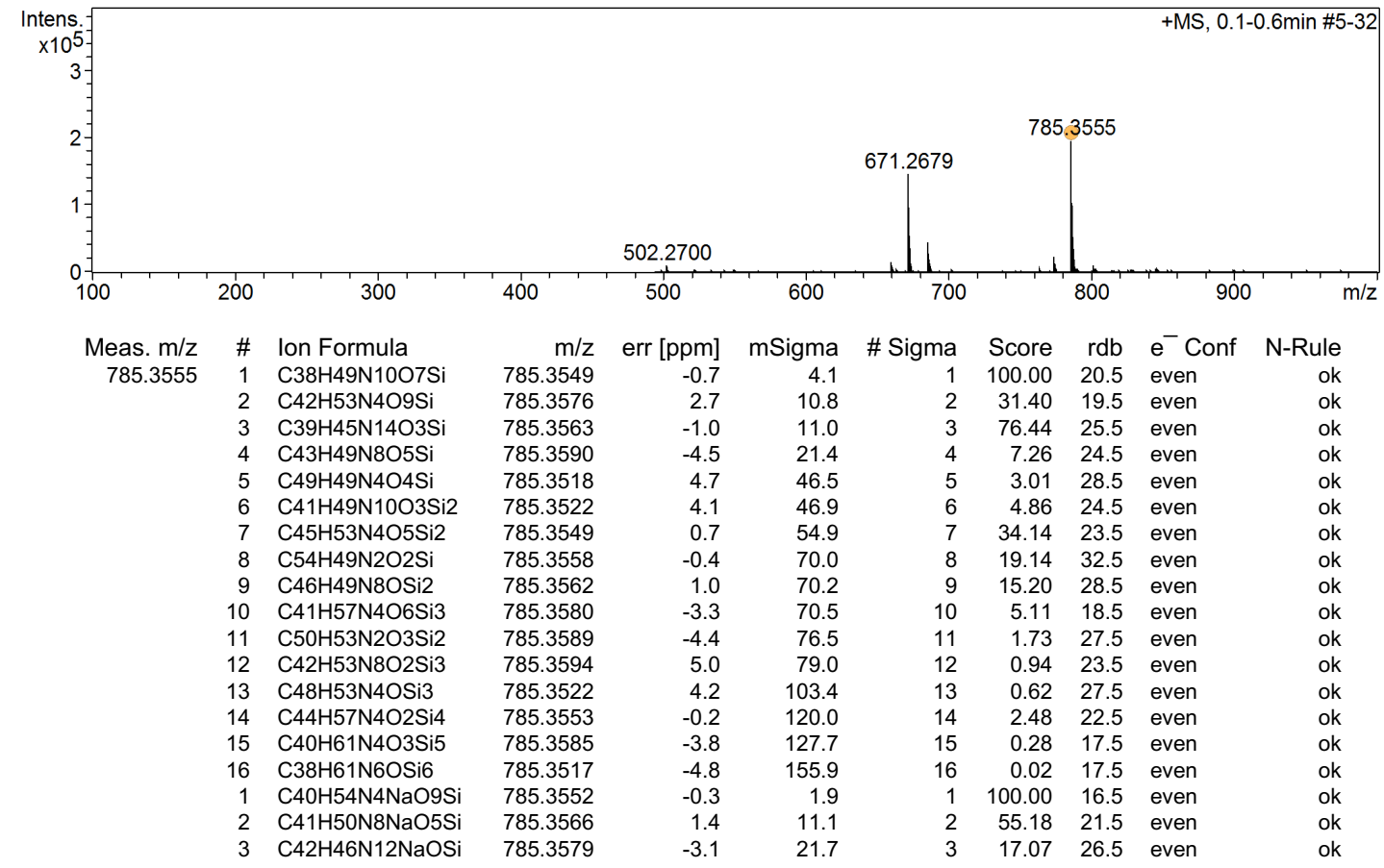

KSJ012-1.d

Bruker Compass DataAnalysis 4.1 $\quad$ printed: 7/11/2019 4:01:40 PM $\quad$ by: lee 1 of 2 
${ }^{1} \mathrm{H}-\mathrm{NMR}$ of Compound $10 \mathrm{~b}\left(500 \mathrm{MHz}, \mathrm{CDCl}_{3}\right)$

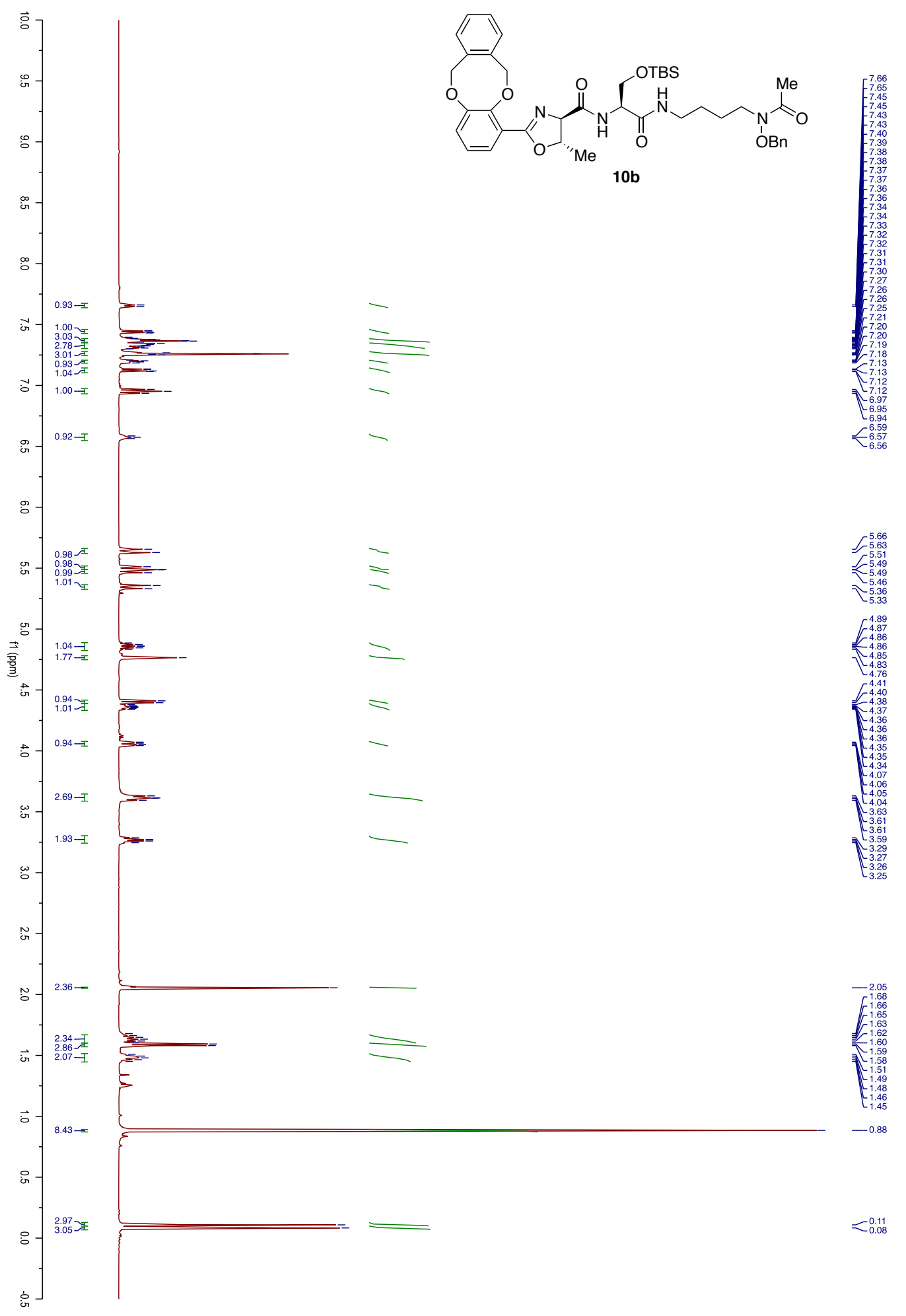


${ }^{13} \mathrm{C}-\mathrm{NMR}$ of Compound 10b (125 $\left.\mathrm{MHz}, \mathrm{CDCl}_{3}\right)$

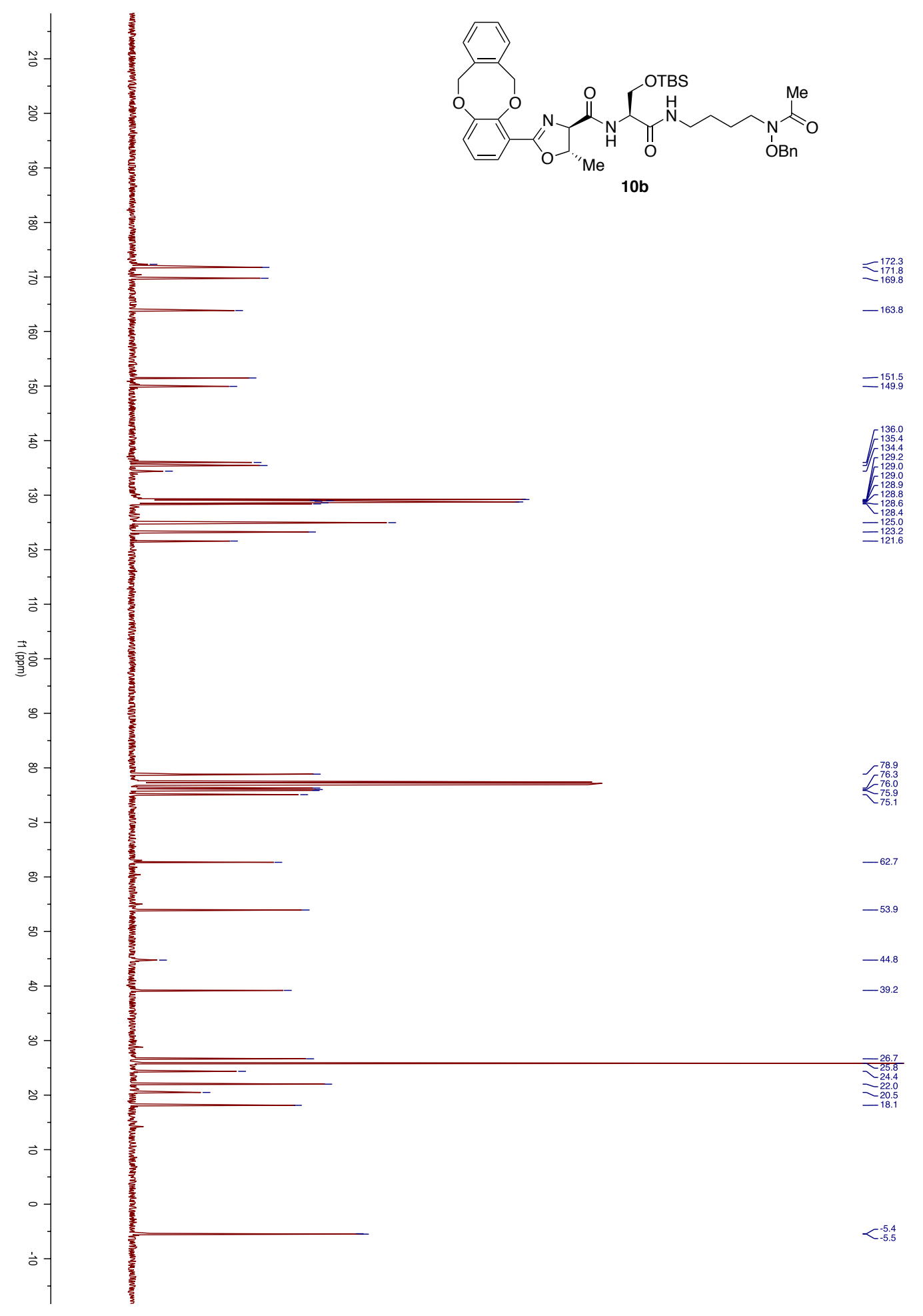


HR-MS of Compound 10b

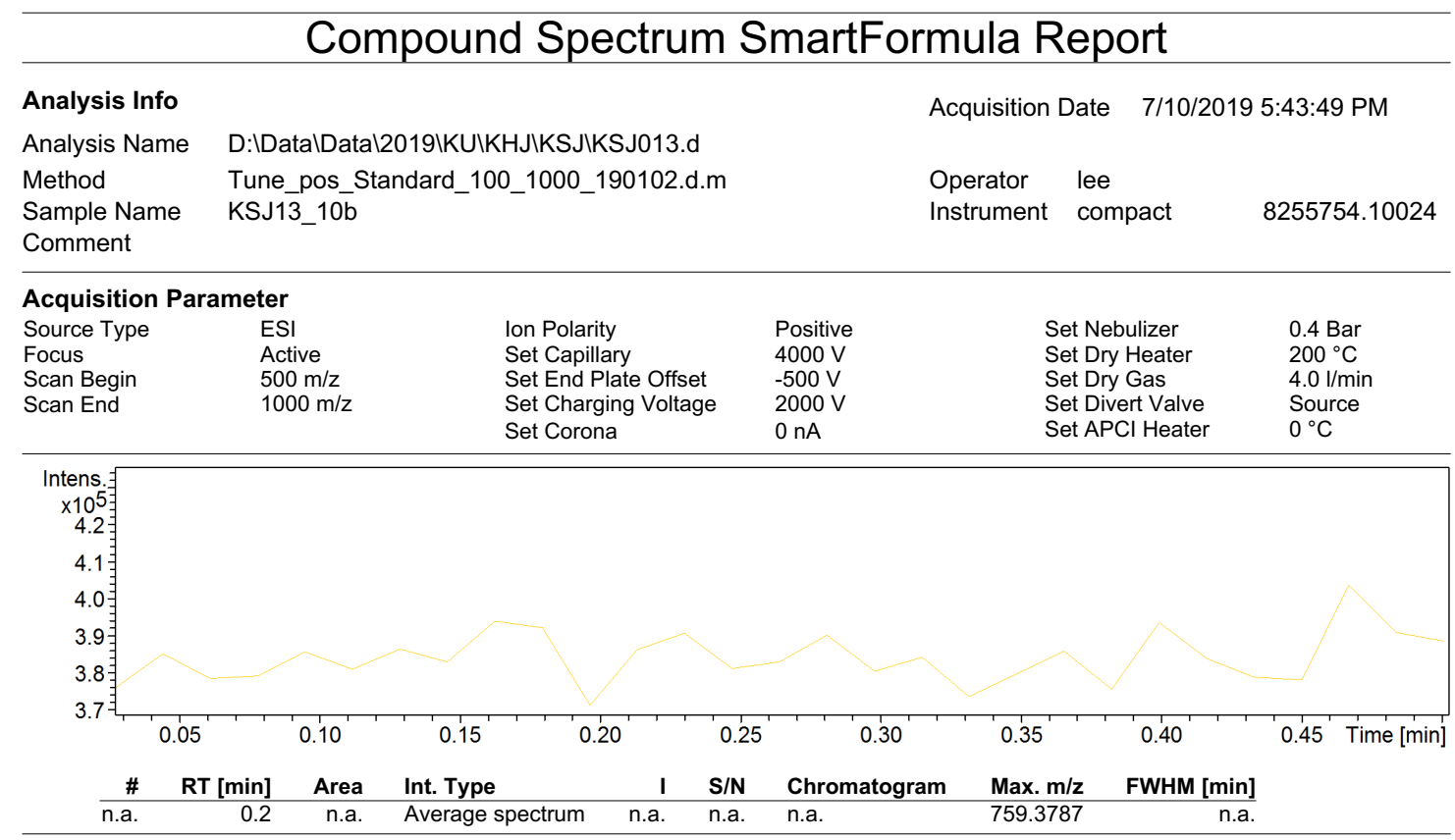

+MS, 0.1-0.4min \#4-24

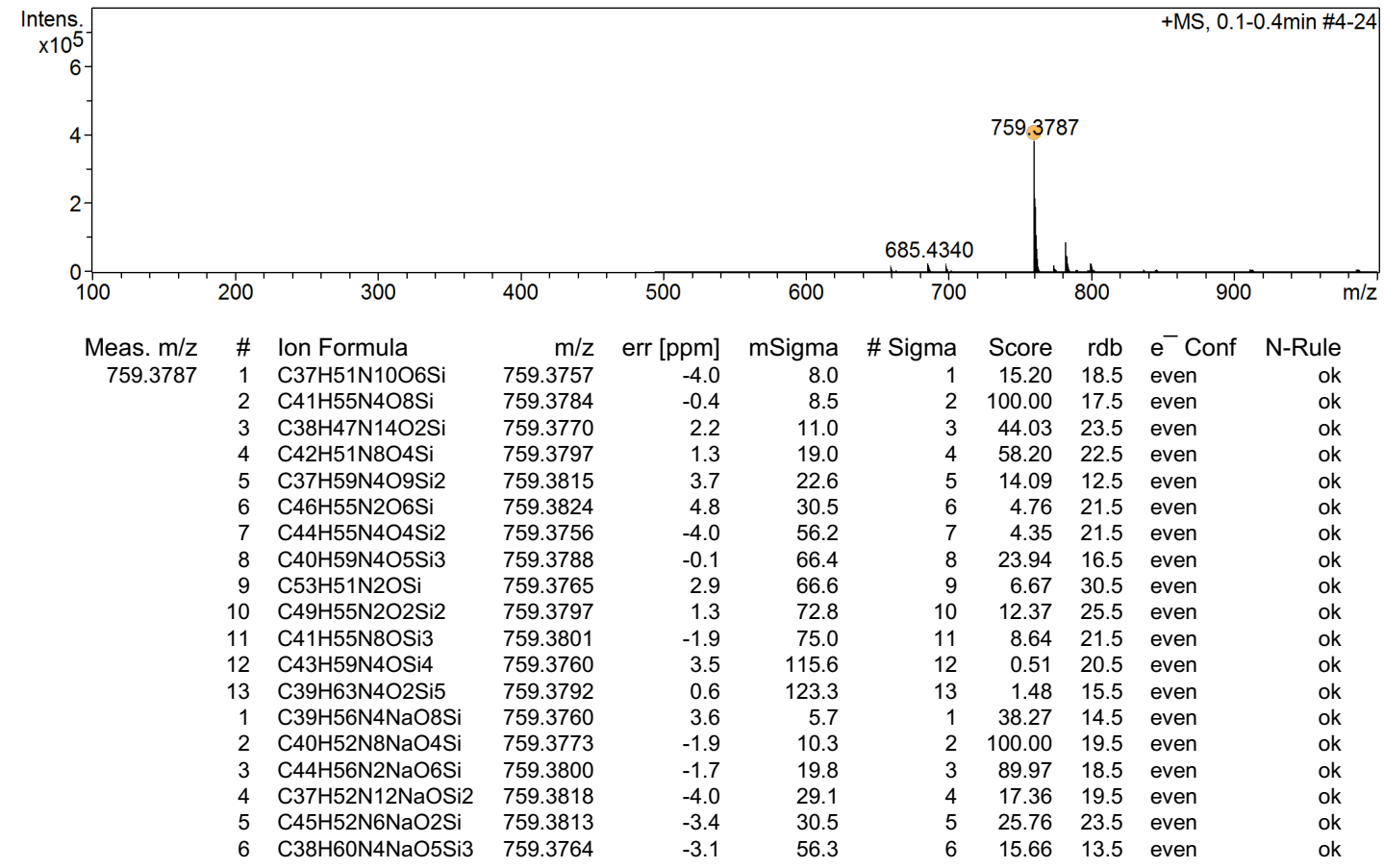

KSJ013.d

Bruker Compass DataAnalysis 4.1 $\quad$ printed: 7/11/2019 4:02:59 PM $\quad$ by: lee 1 of 2 
${ }^{1} \mathrm{H}-\mathrm{NMR}$ of Compound 1a-ii (500 MHz, $\left.\mathrm{CD}_{3} \mathrm{OD}\right)$

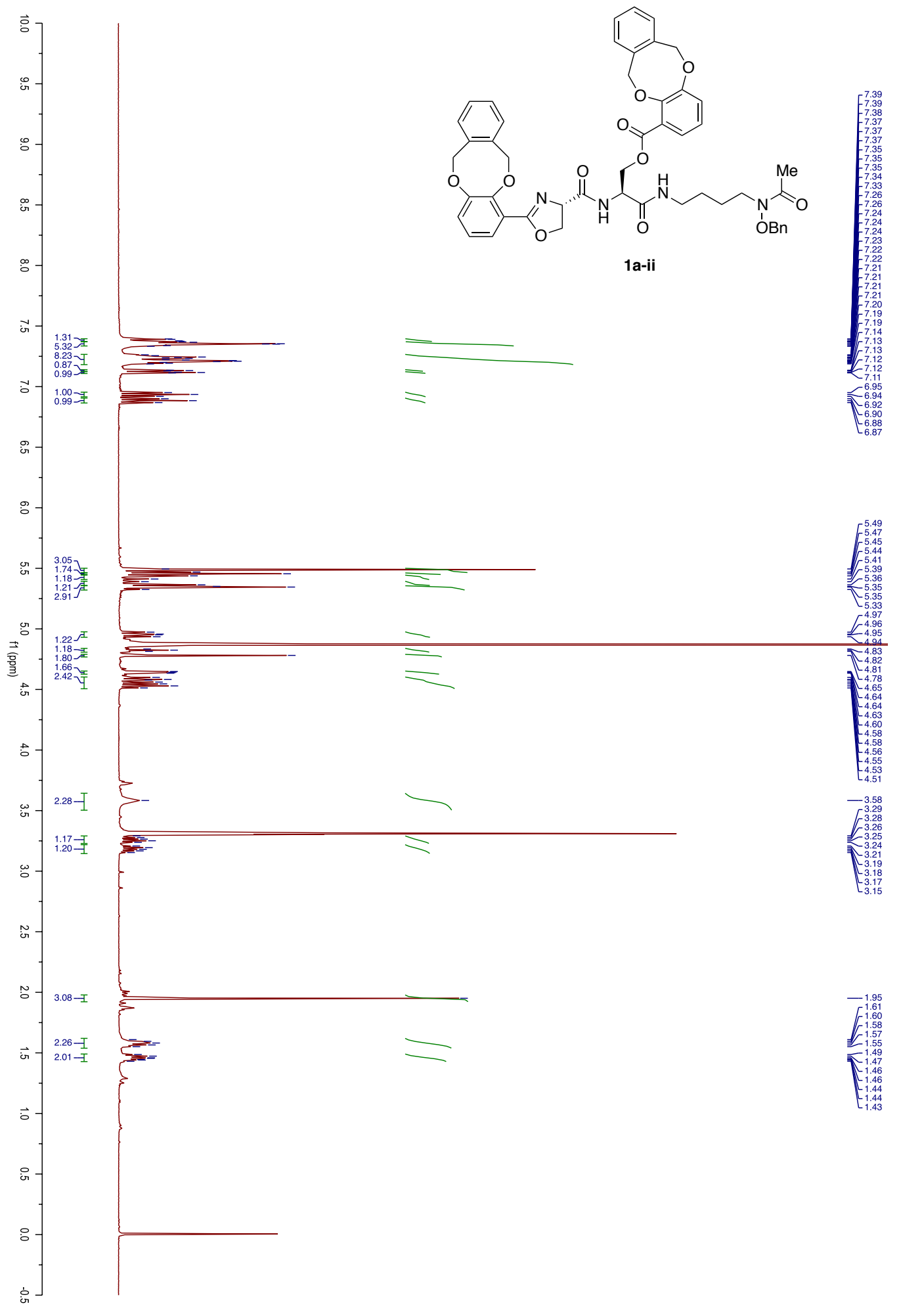




\section{${ }^{13} \mathrm{C}-\mathrm{NMR}$ of Compound 1a-ii $\left(\mathrm{CD}_{3} \mathrm{OD}\right)$}

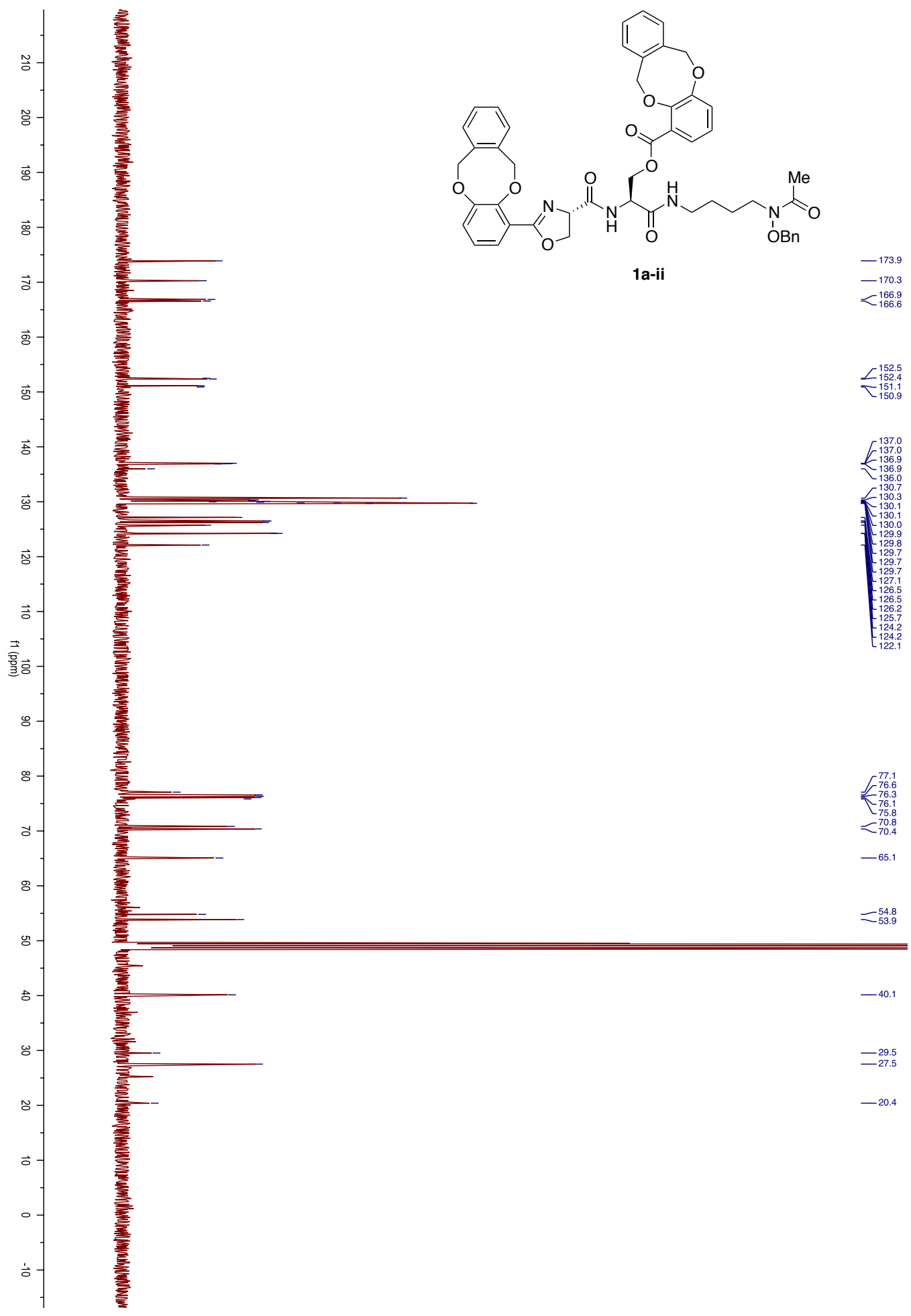


HR-MS of Compound 1a-ii

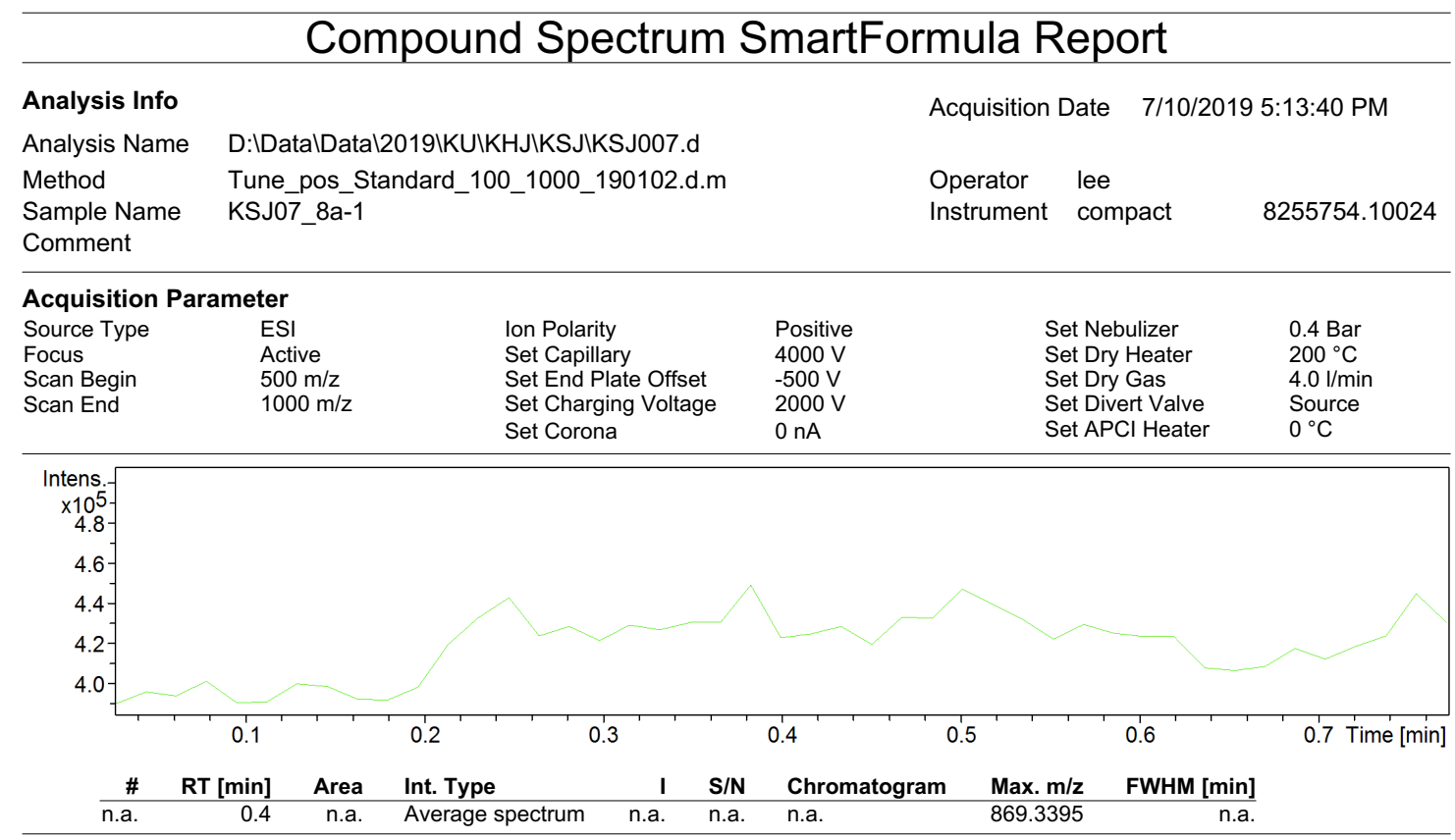

+MS, 0.2-0.6min \#11-37

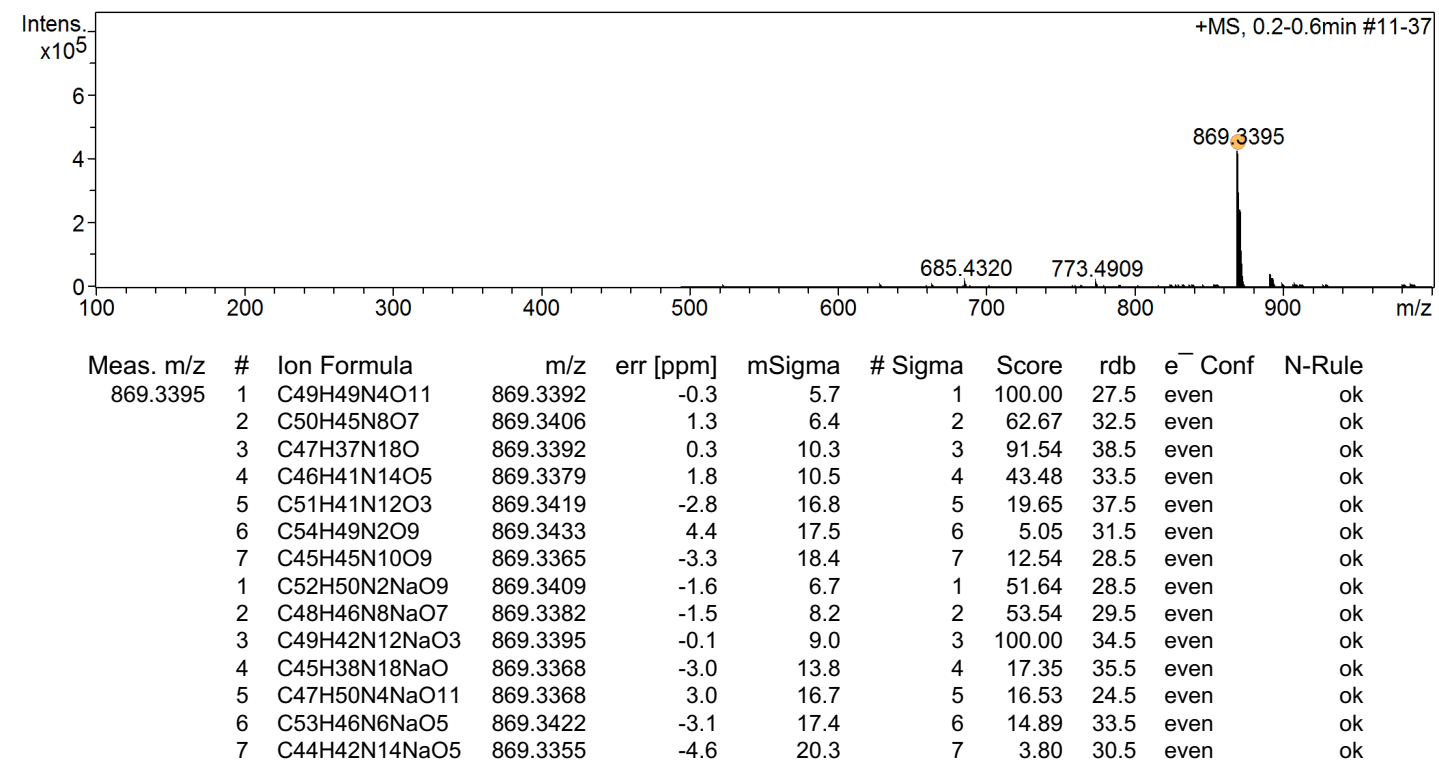

KSJ007.d

Bruker Compass DataAnalysis 4.1 $\quad$ printed: 7/11/2019 4:06:48 PM $\quad$ by: lee 1 Page 1 ef 
${ }^{1}$ H-NMR of Compound 1a (Fimsbactin A, 500 MHz, DMSO-d ${ }^{6}$ )

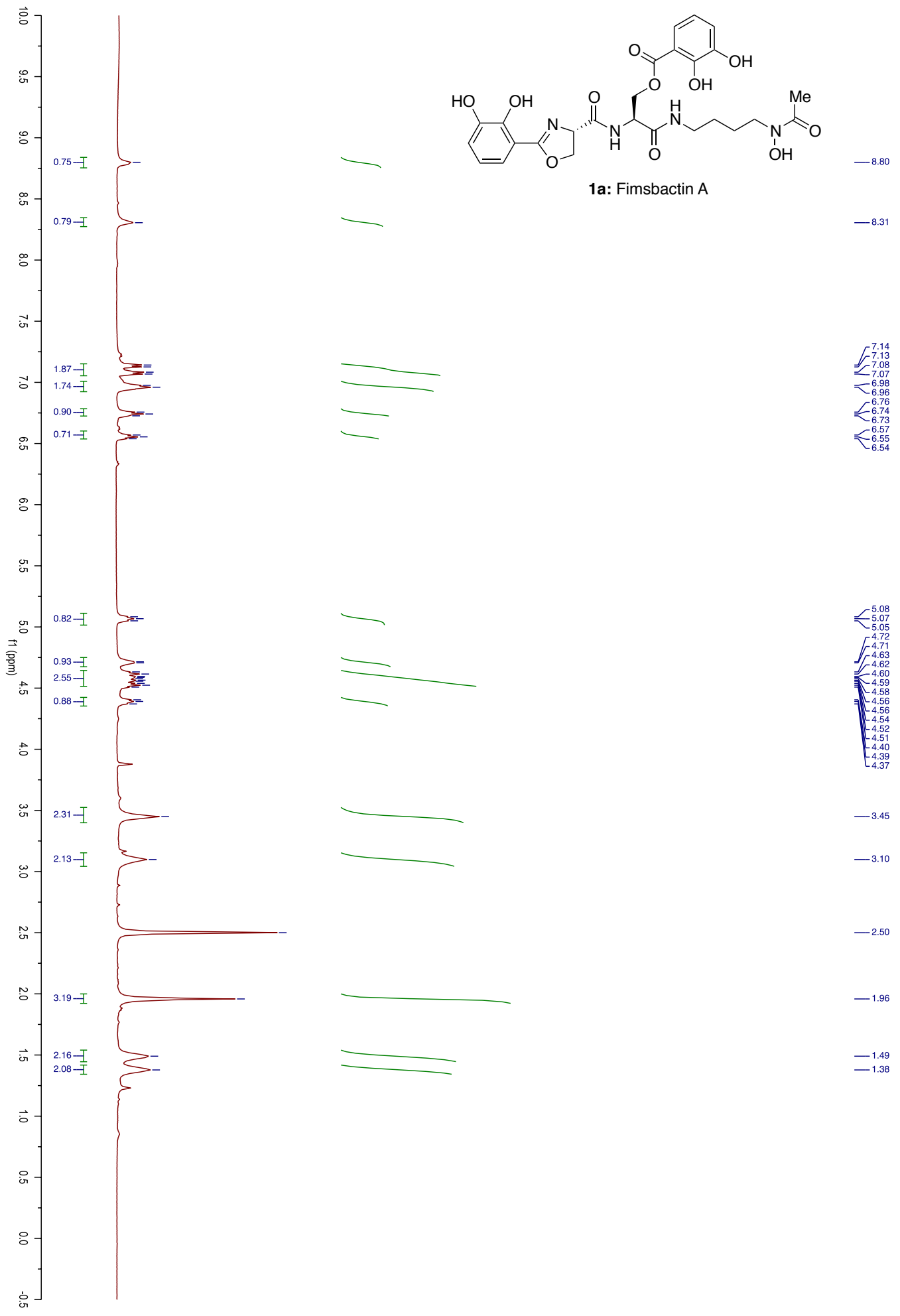




\section{${ }^{13}$ C-NMR of Compound 1a (Fimsbactin A, 125 MHz, DMSO-d ${ }^{6}$ )}

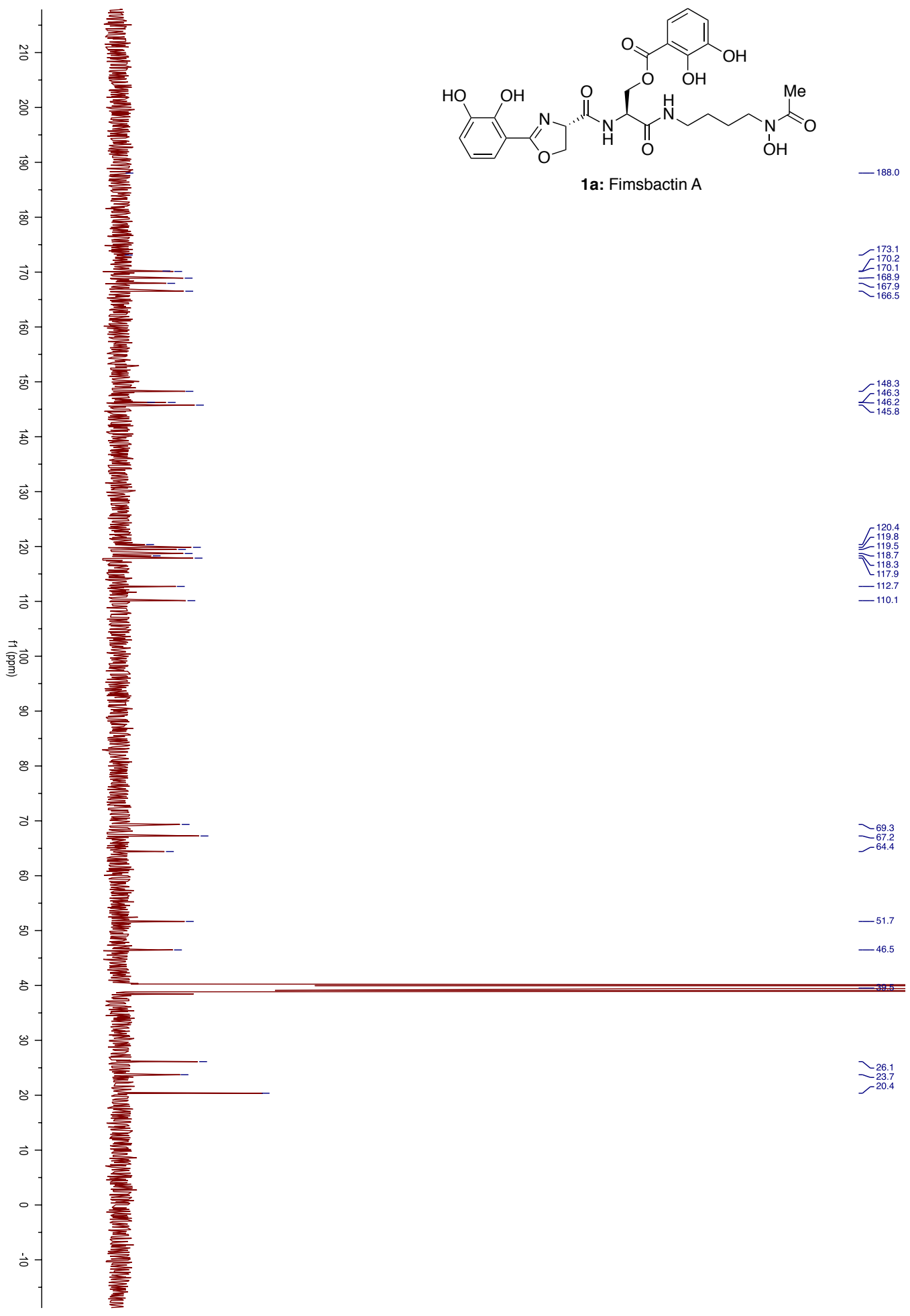




\section{HR-MS of Compound 1a (Fimsbactin A)}

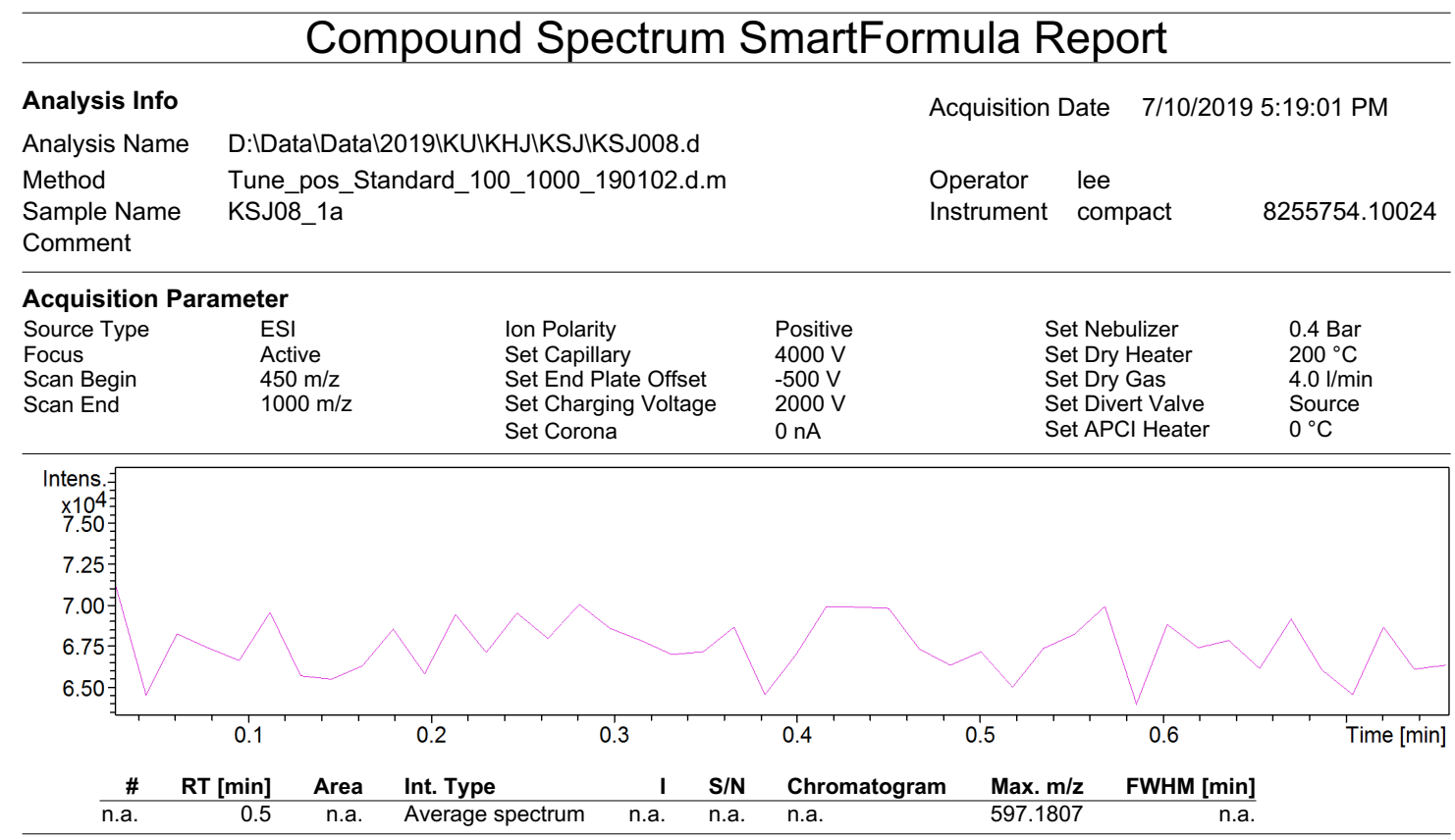

+MS, 0.3-0.7min \#16-42

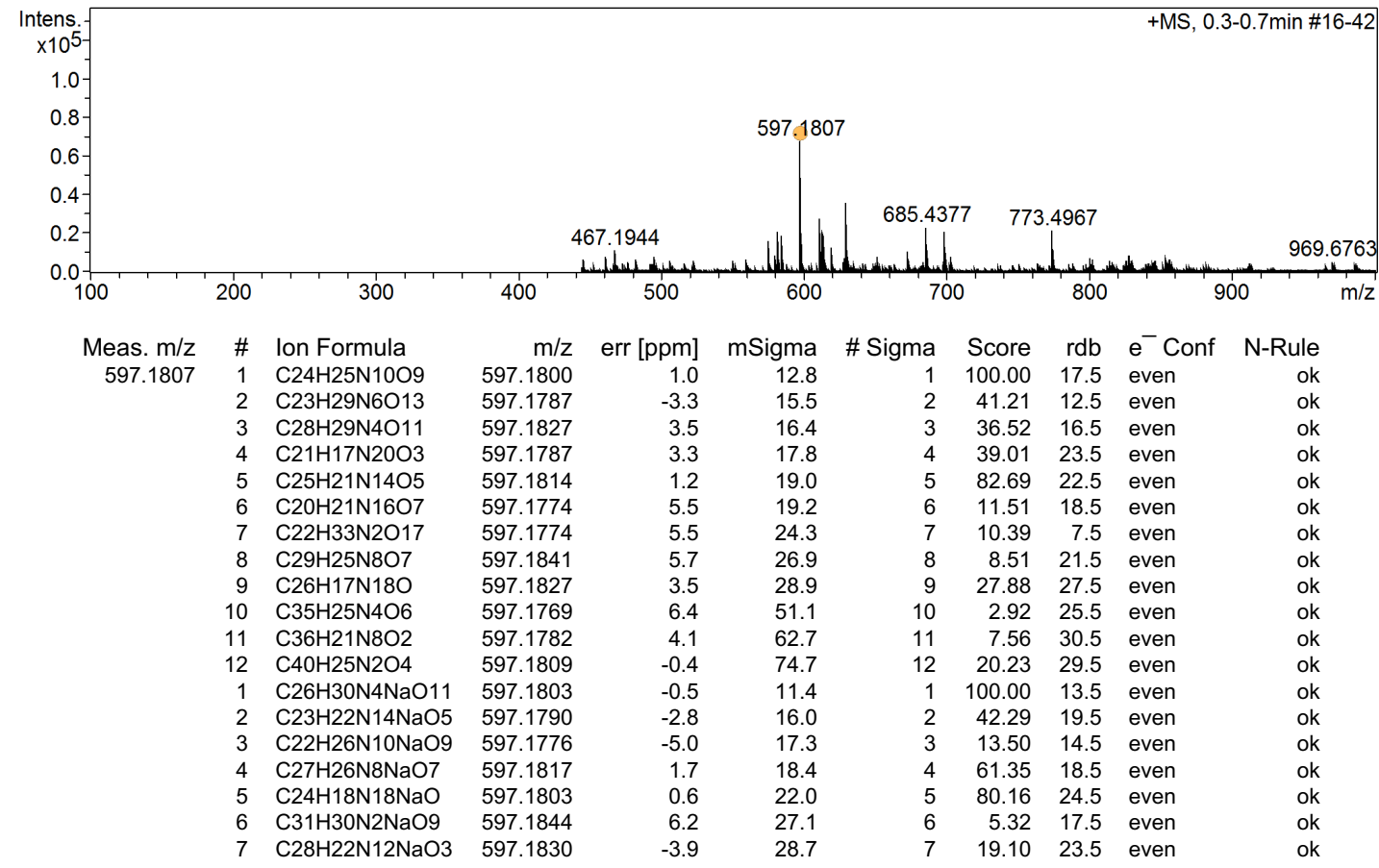

KSJ008.d

Bruker Compass DataAnalysis 4.1 $\quad$ printed: 7/11/2019 3:55:18 PM $\quad$ by: lee 1 of 2 
${ }^{1} \mathrm{H}-\mathrm{NMR}$ of Compound epi-1a-ii (500 MHz, $\left.\mathrm{CD}_{3} \mathrm{OD}\right)$

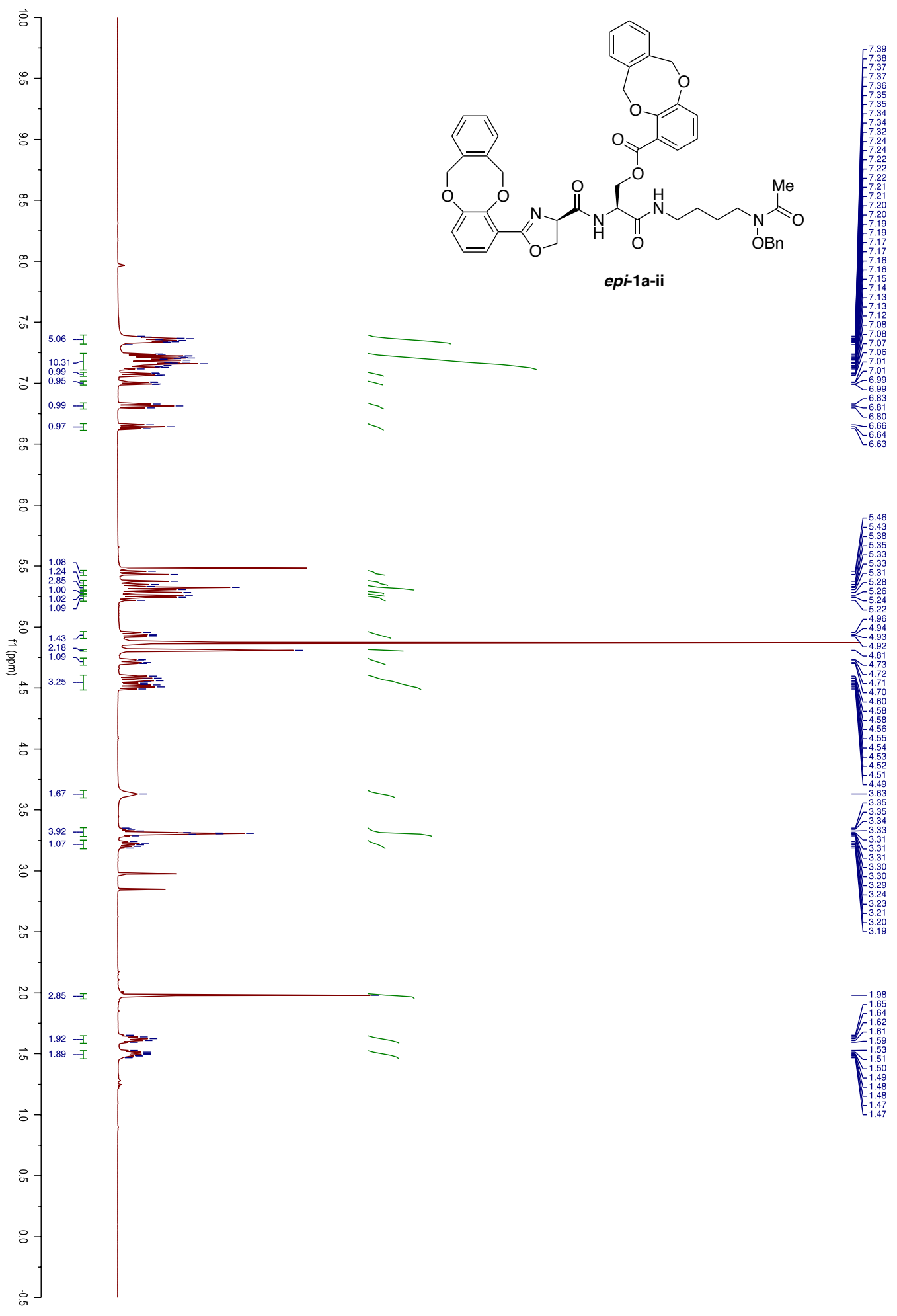




\section{${ }^{13}$ C-NMR of Compound epi-1a-ii}

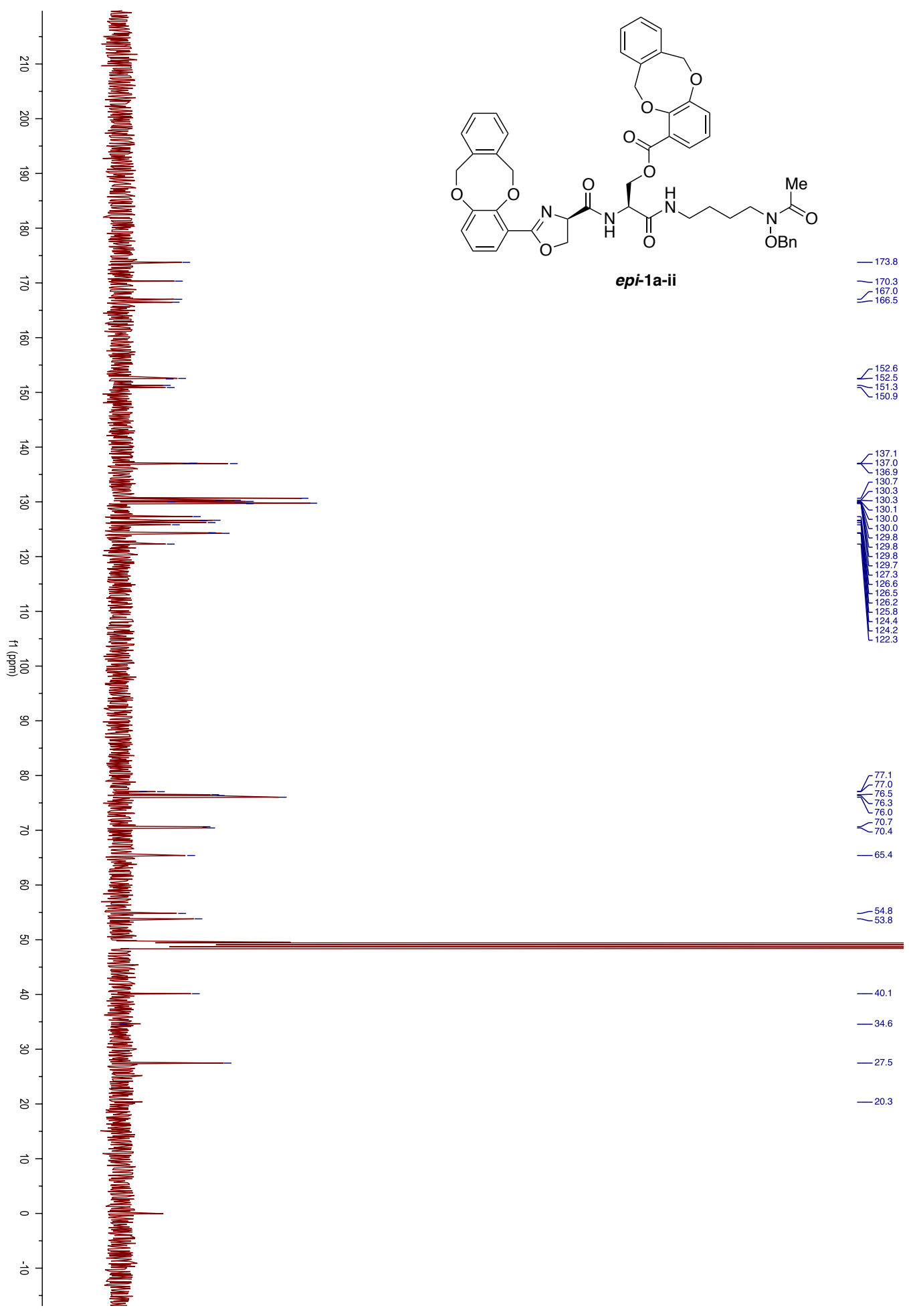




\section{HR-MS of Compound epi-1a-ii}

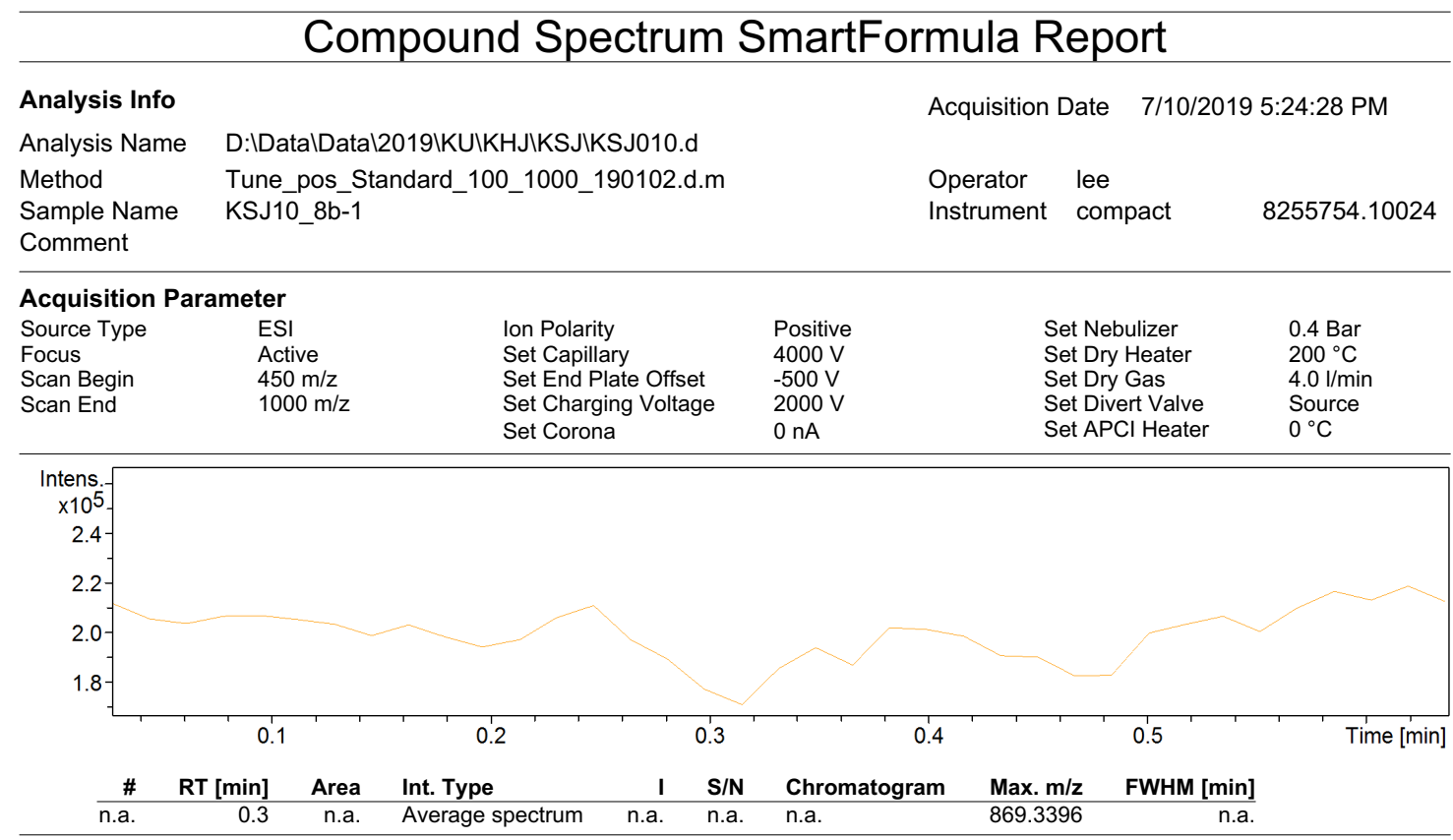

+MS, 0.1-0.5min \#7-31

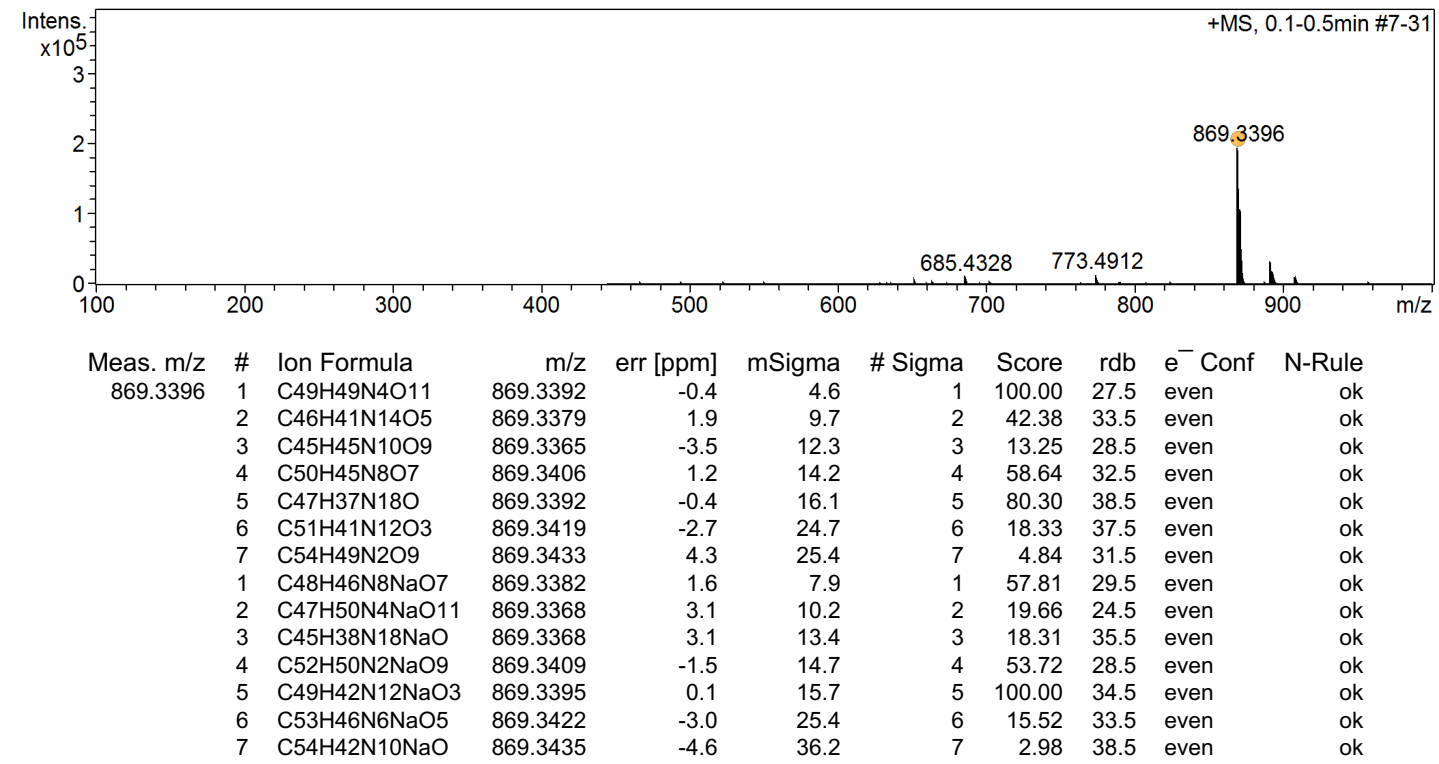

KSJ010.d

Bruker Compass DataAnalysis 4.1 $\quad$ printed: 7/11/2019 3:58:37 PM $\quad$ by: lee 1 Page 1 ef 
${ }^{1} \mathrm{H}-\mathrm{NMR}$ of Compound epi-1a (epi-Fimsbactin A, $500 \mathrm{MHz}, \mathrm{DMSO}-\mathrm{d}^{6}$ )

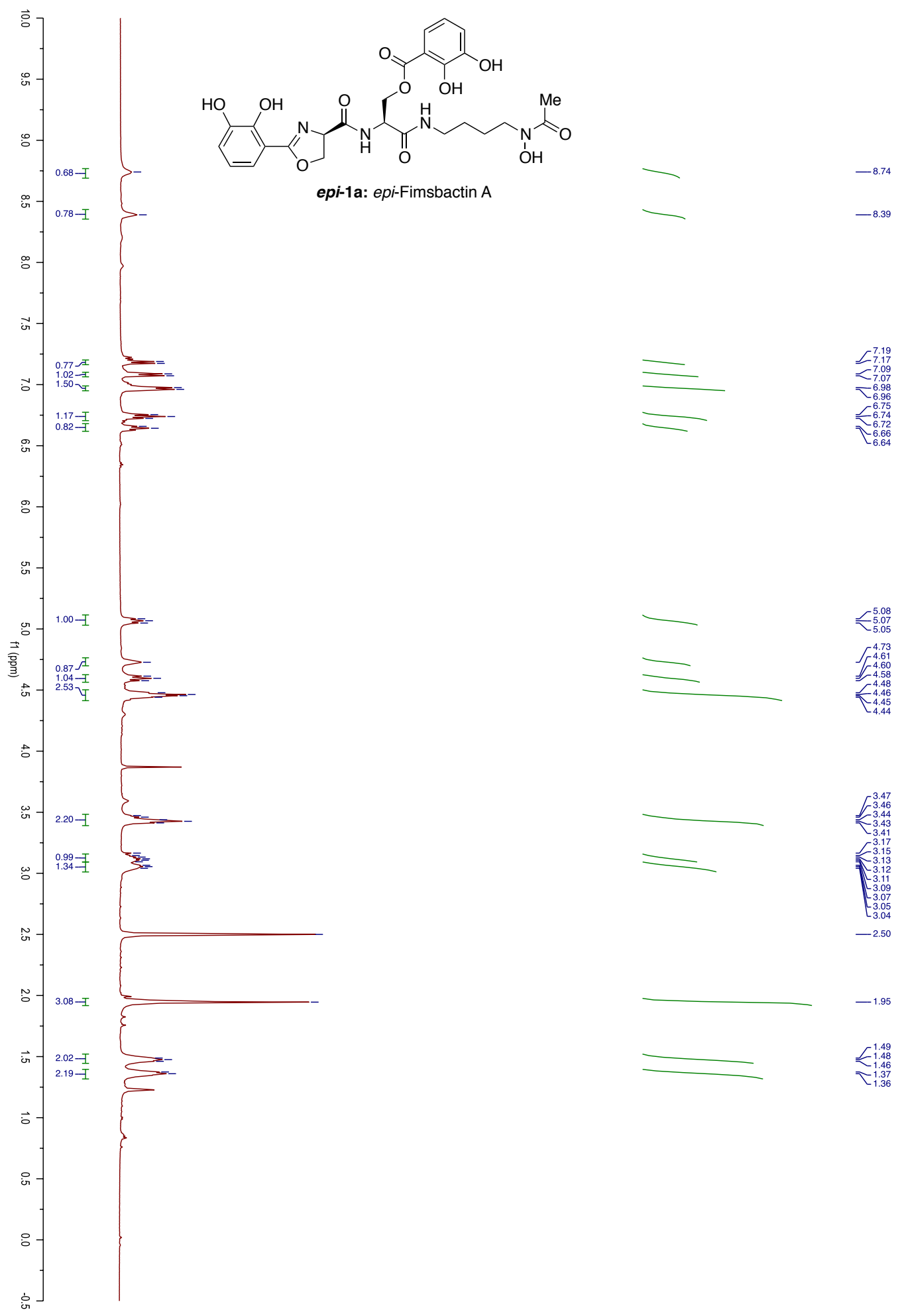


${ }^{13}$ C-NMR of Compound epi-1a (epi-Fimsbactin A, 125 MHz, DMSO-d ${ }^{6}$ )

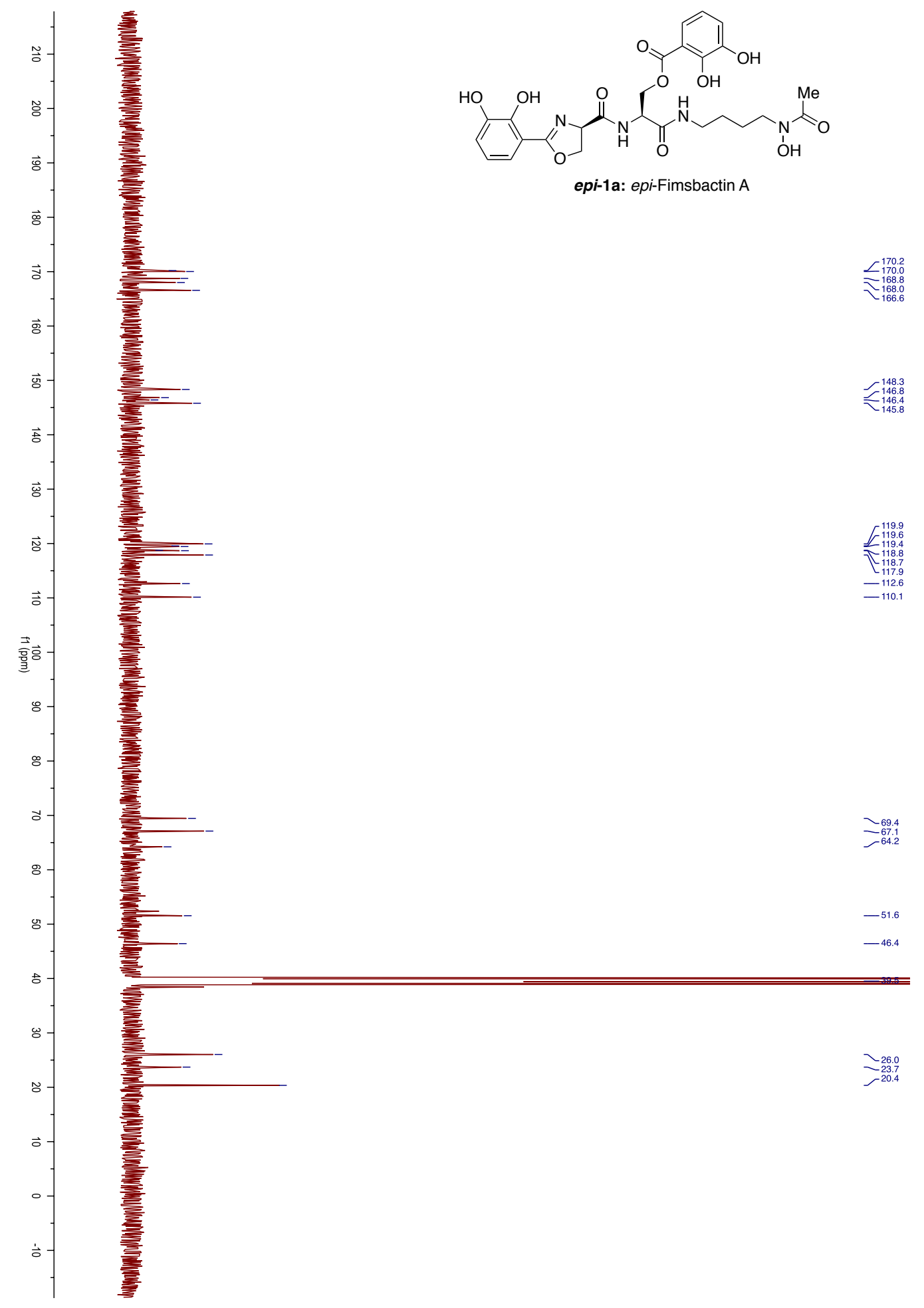




\section{HR-MS of Compound epi-1a (epi-Fimsbactin A)}

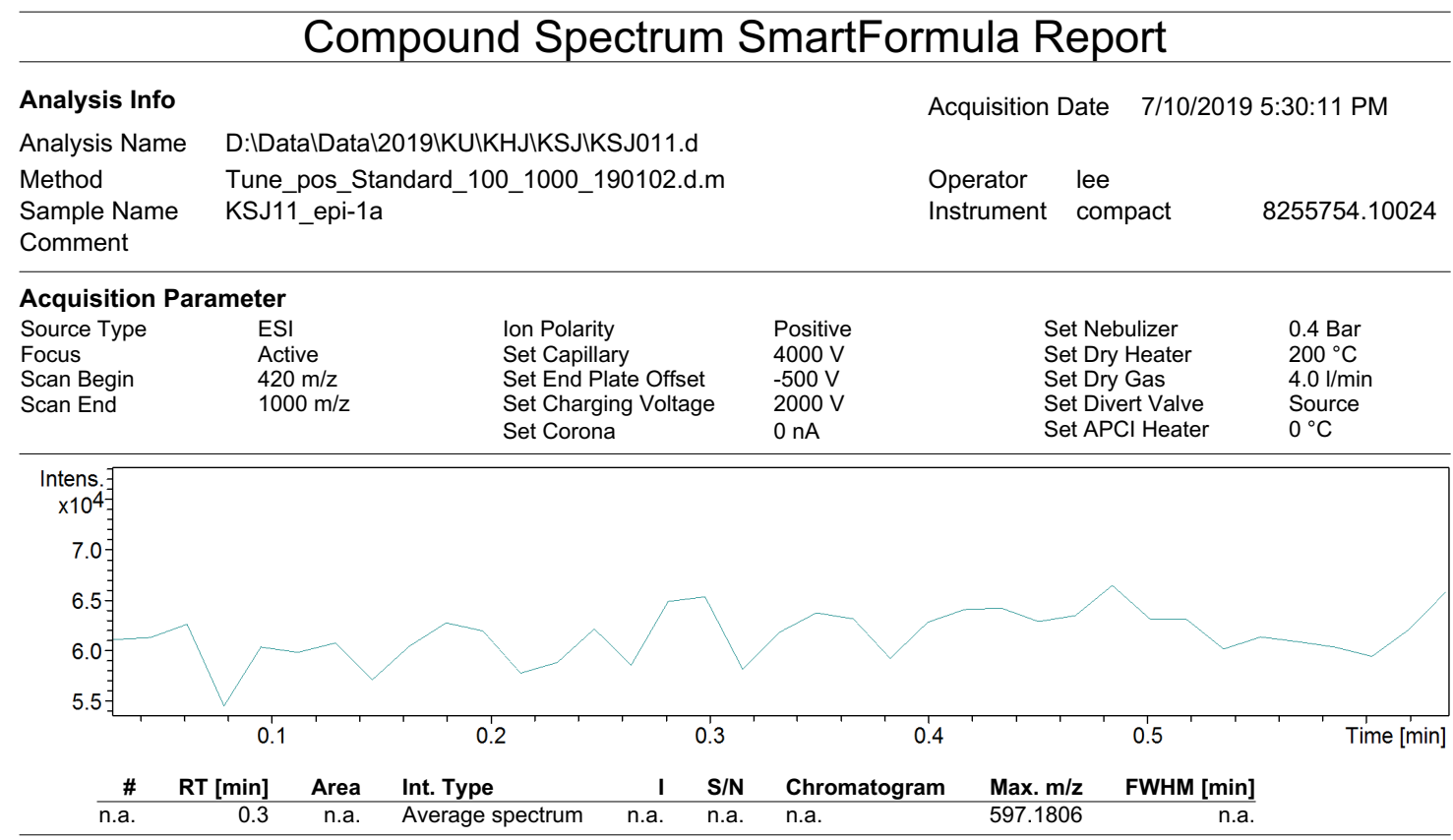

+MS, 0.1-0.5 $\min \# 4-29$

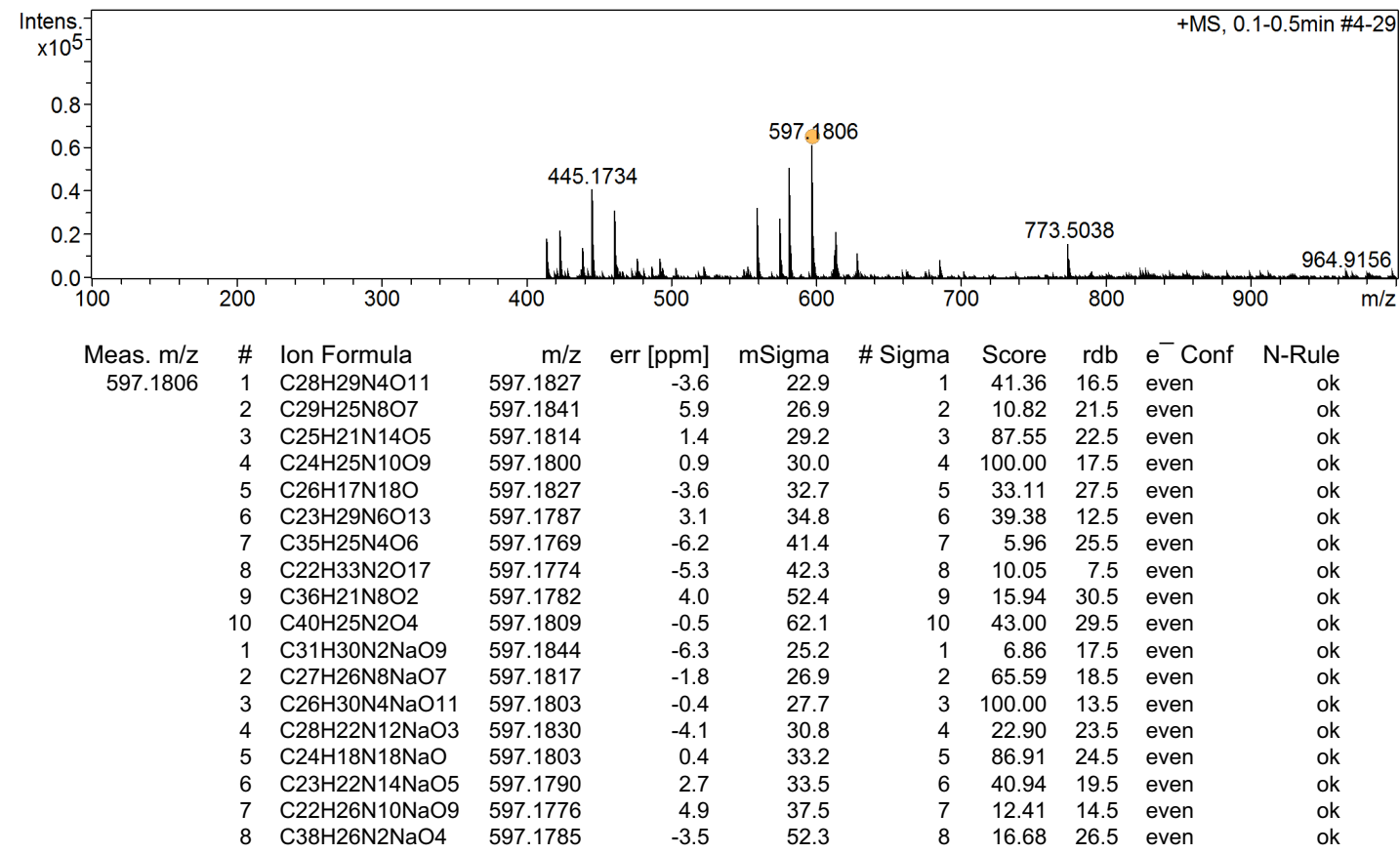

KSJ011.d

Bruker Compass DataAnalysis 4.1 $\quad$ printed: 7/11/2019 4:00:00 PM $\quad$ by: lee 1 Page 1 ef 
${ }^{1} \mathrm{H}-\mathrm{NMR}$ of Compound dia-1b-ii (500 $\mathrm{MHz}, \mathrm{CDCl}_{3}$ )

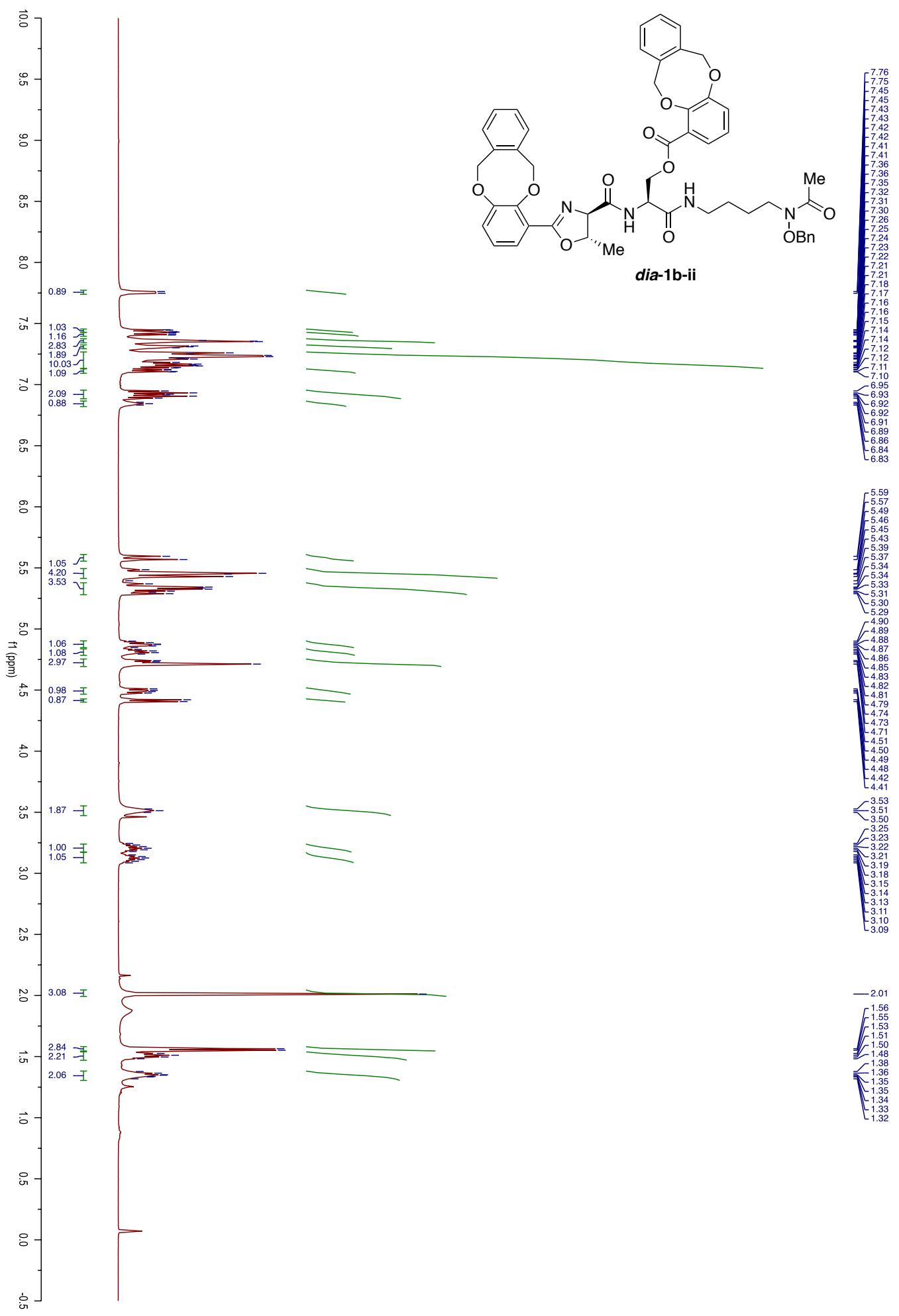


${ }^{13} \mathrm{C}-\mathrm{NMR}$ of Compound dia-1b-ii (125 $\mathrm{MHz}, \mathrm{CDCl}_{3}$ )

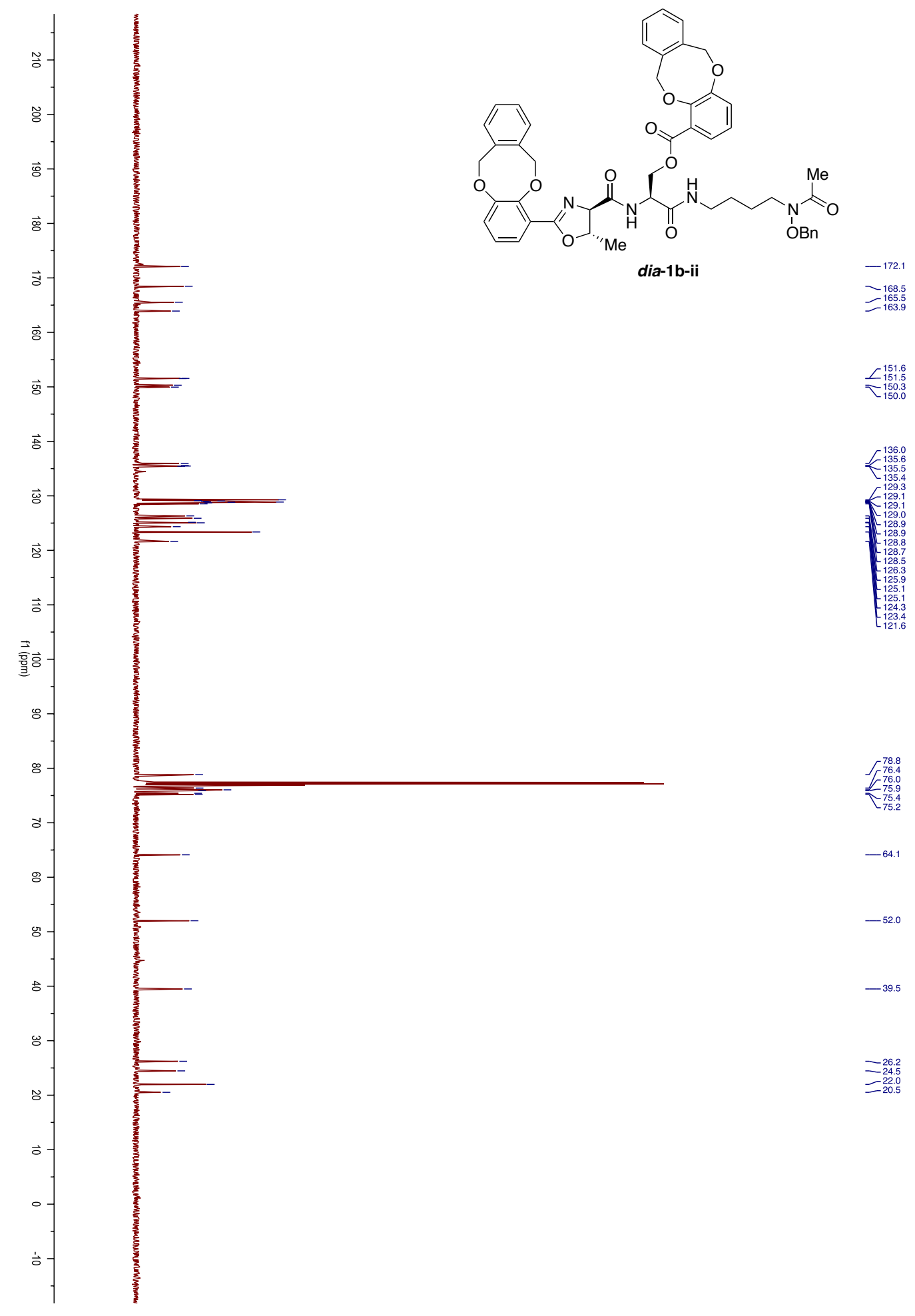




\section{HR-MS of Compound dia-1b-ii}

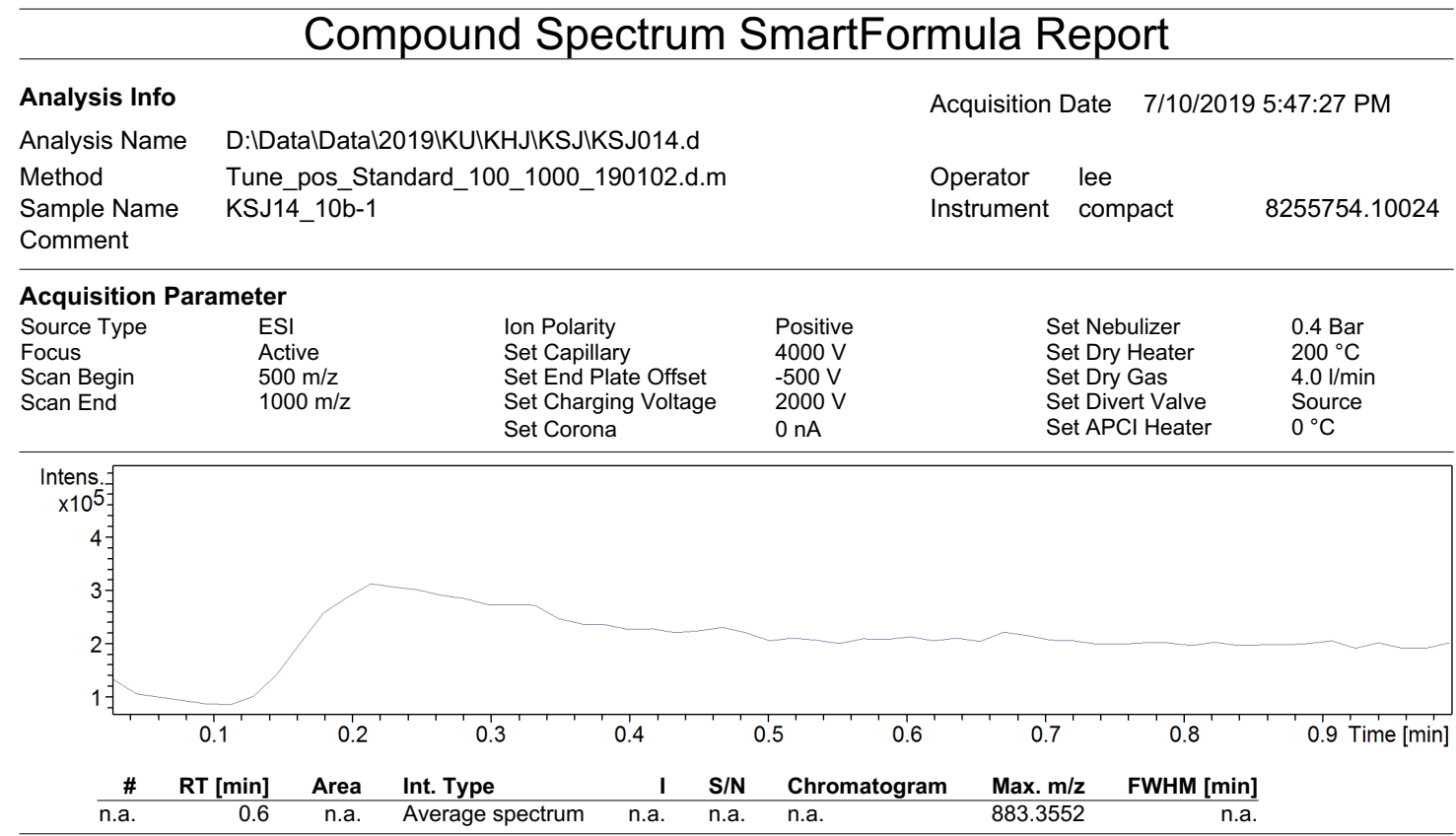

+MS, 0.3-0.9min \#17-54

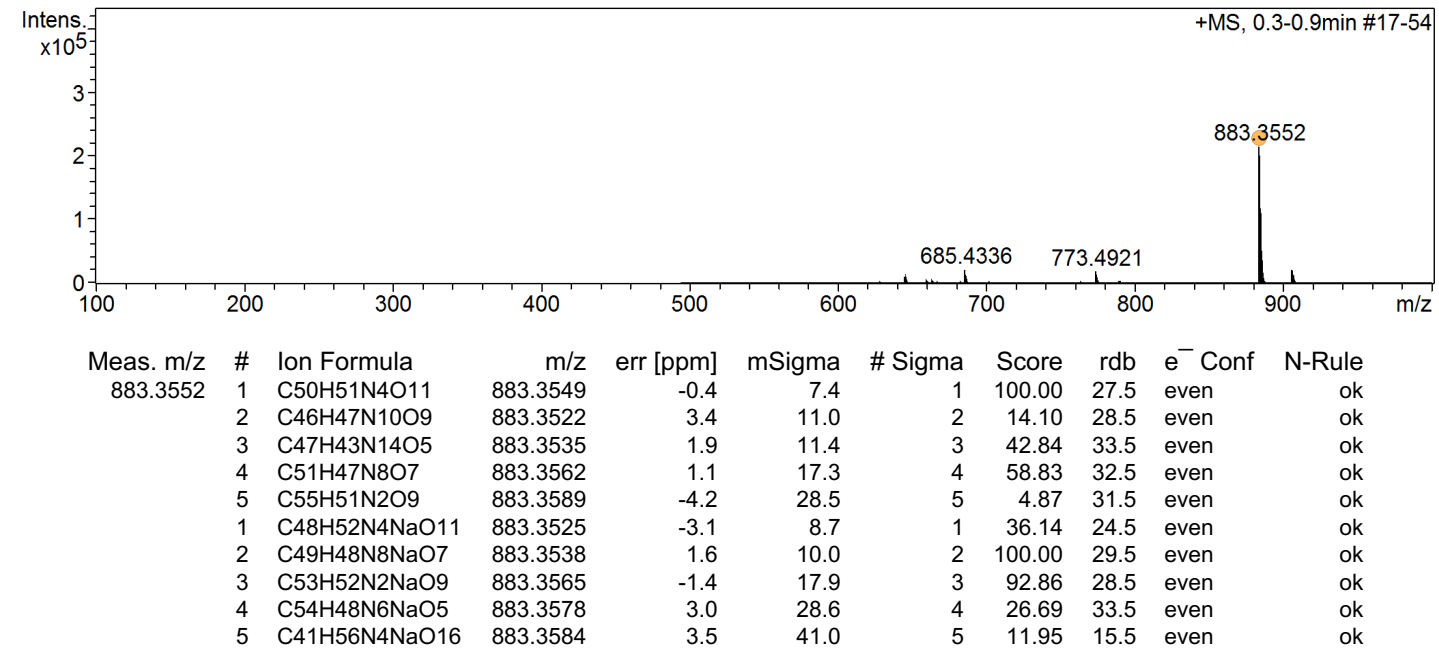

KSJ014.d

Bruker Compass DataAnalysis 4.1 $\quad$ printed: 7/11/2019 4:04:33 PM $\quad$ by: lee 1 Page 1 ef


${ }^{1}$ H-NMR of Compound dia-1b (dia-Fimsbactin B, 500 MHz, DMSO-d ${ }^{6}$ )

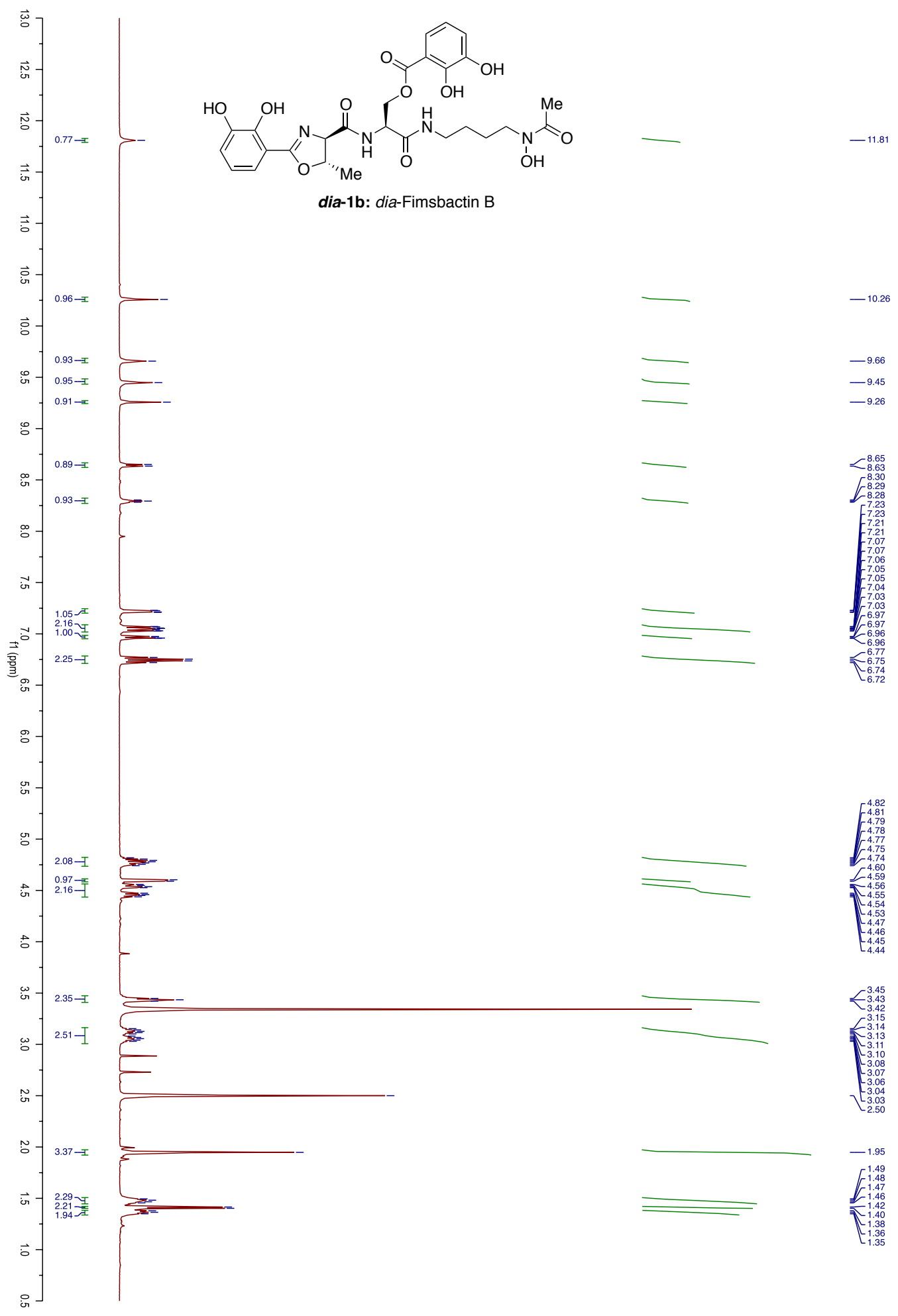


${ }^{13}$ C-NMR of Compound dia-1b (dia-Fimsbactin B, 125 MHz, DMSO-d ${ }^{6}$ )

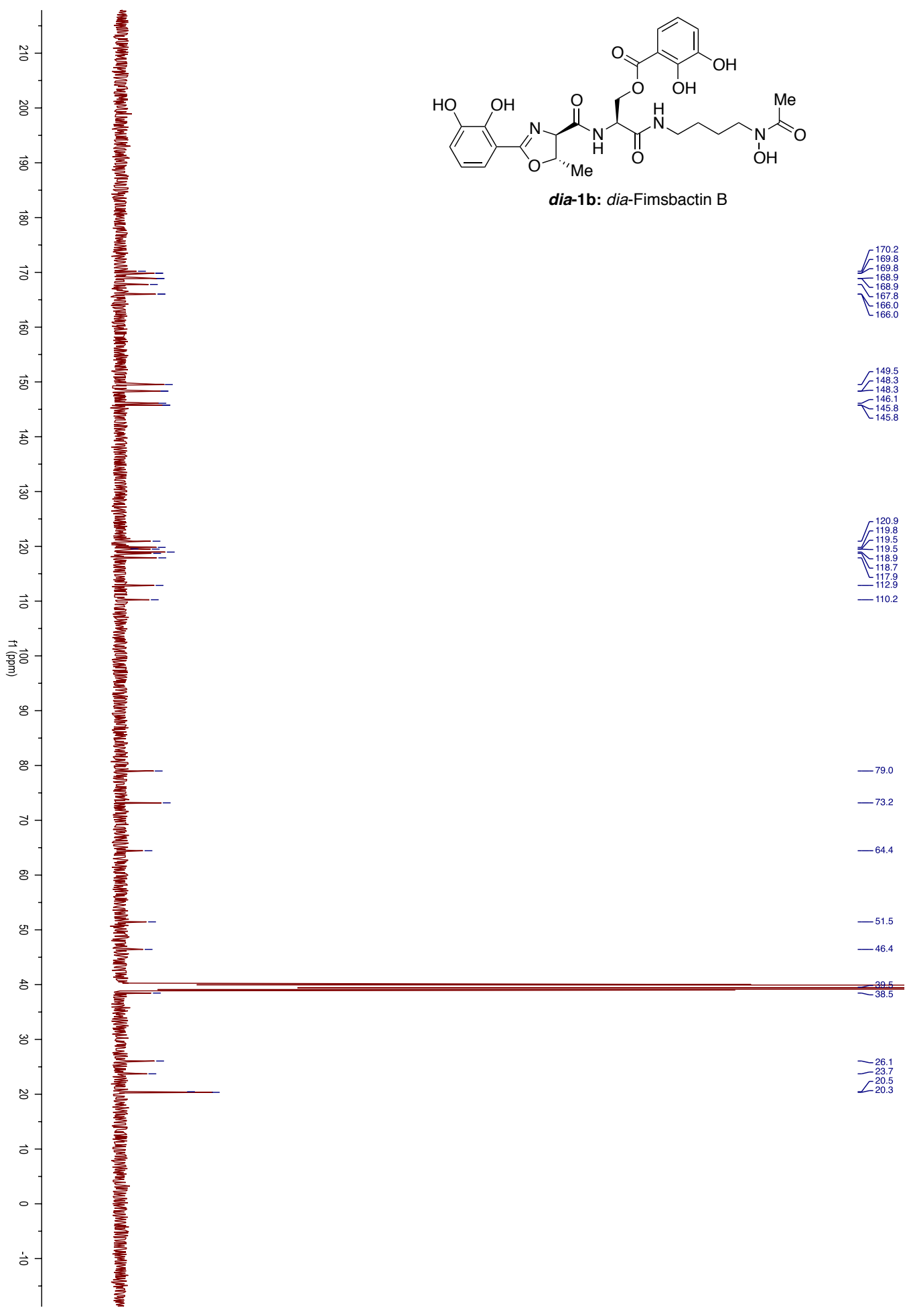




\section{HR-MS of Compound dia-1b (dia-Fimsbactin B)}

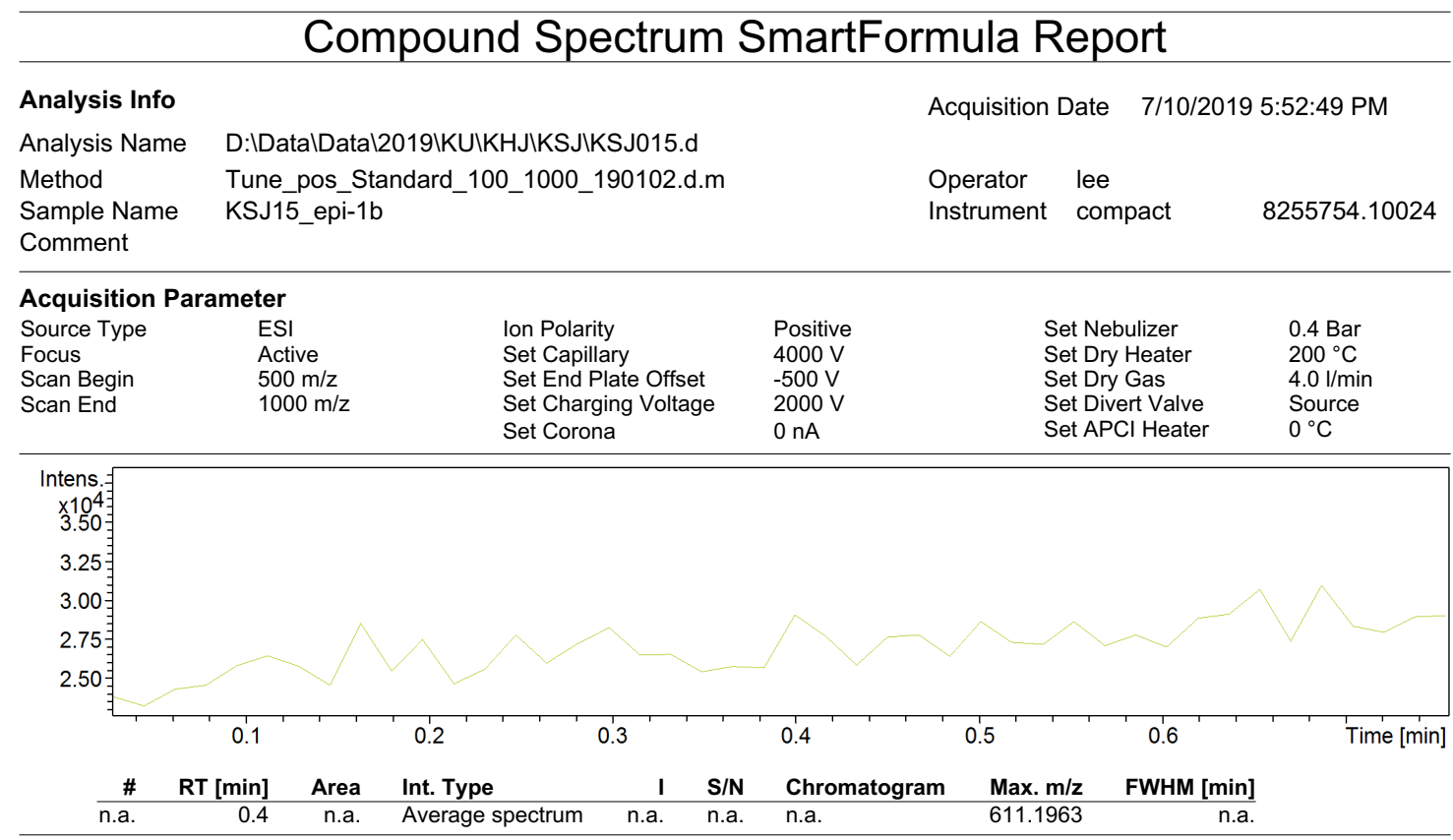

+MS, 0.2-0.6min \#11-35

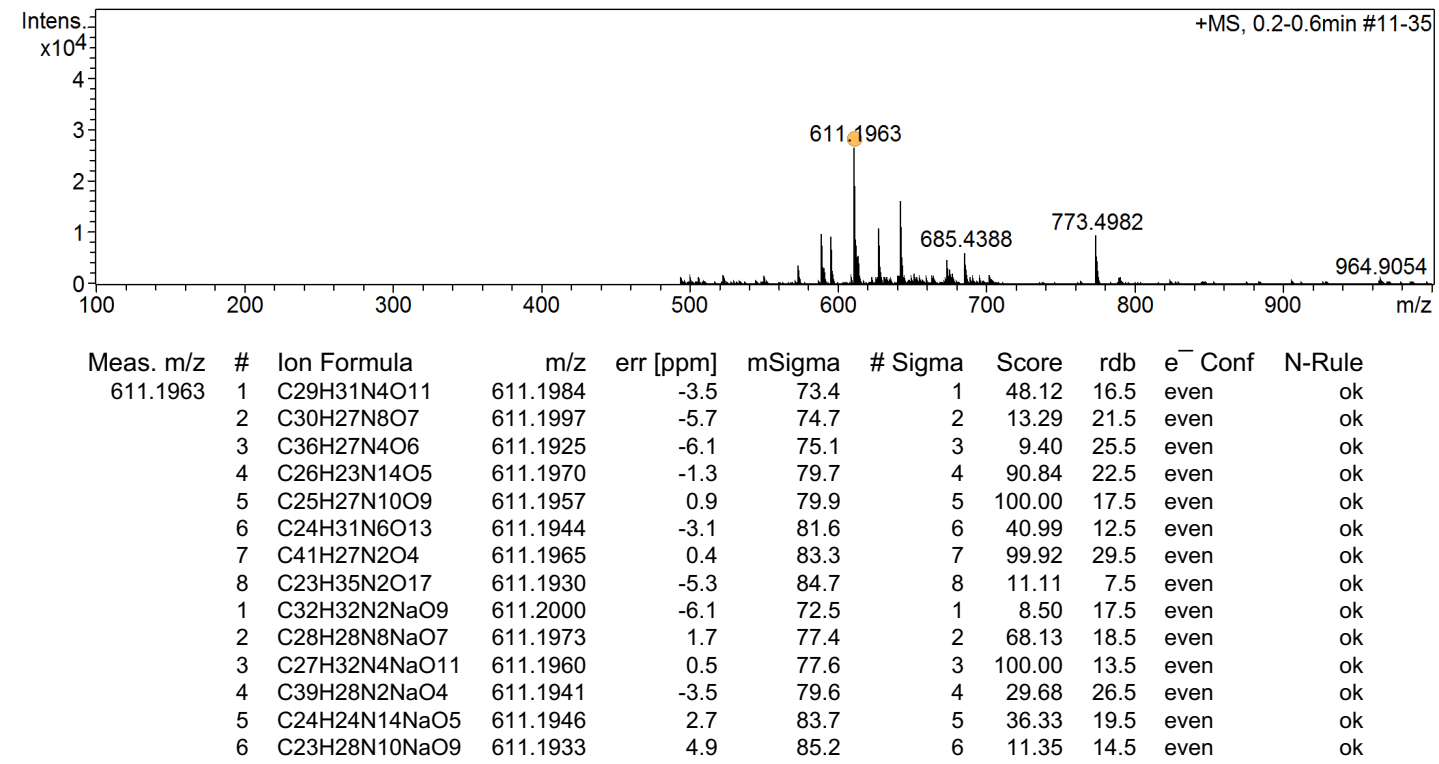

KSJ015.d

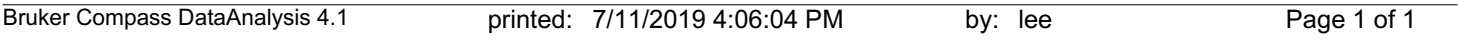

